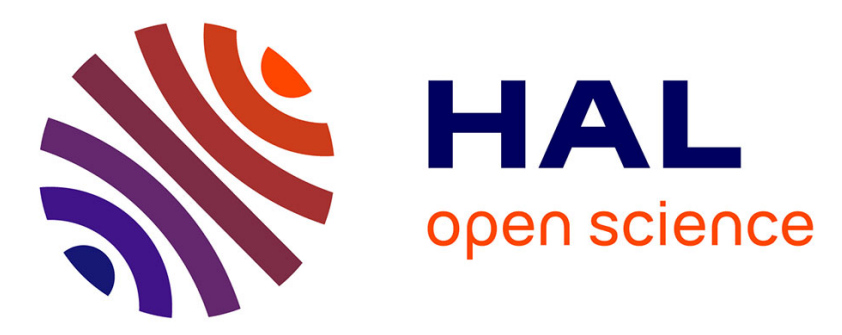

\title{
Diffusion Across Semi-permeable Barriers: Spectral Properties, Efficient Computation, and Applications
}

Nicolas Moutal, Denis S Grebenkov

\section{To cite this version:}

Nicolas Moutal, Denis S Grebenkov. Diffusion Across Semi-permeable Barriers: Spectral Properties, Efficient Computation, and Applications. Journal of Scientific Computing, 2019, 81 (3), pp.1630-1654. 10.1007/s10915-019-01055-5 . hal-02988851

\section{HAL Id: hal-02988851 https://hal.science/hal-02988851}

Submitted on 4 Nov 2020

HAL is a multi-disciplinary open access archive for the deposit and dissemination of scientific research documents, whether they are published or not. The documents may come from teaching and research institutions in France or abroad, or from public or private research centers.
L'archive ouverte pluridisciplinaire HAL, est destinée au dépôt et à la diffusion de documents scientifiques de niveau recherche, publiés ou non, émanant des établissements d'enseignement et de recherche français ou étrangers, des laboratoires publics ou privés. 


\title{
Diffusion across semi-permeable barriers: spectral properties, efficient computation, and applications
}

\author{
Nicolas Moutal • Denis Grebenkov
}

the date of receipt and acceptance should be inserted later

\begin{abstract}
We present an efficient method to compute the eigenvalues and eigenmodes of the diffusion operator $\nabla(D \nabla)$ on one-dimensional heterogeneous structures with multiple semi-permeable barriers. This method allows us to calculate the diffusion propagator and related quantities such as diffusion MRI signal or first exit time distribution analytically for regular geometries and numerically for arbitrary ones. The effect of the barriers and the transition from infinite permeability (no barriers) to zero permeability (impermeable barriers) are investigated.
\end{abstract}

Keywords Diffusion · Semi-permeable barriers · Laplacian spectrum · Multilayer · Composite medium · Diffusion MRI · First-passage phenomena

\section{Introduction}

Diffusion is a very broad transport mechanism which may describe heat conduction in solids as well as molecular exchanges in biological systems, among many examples. One often characterizes diffusion processes by the "diffusion propagator" (or "heat kernel") $G\left(x_{0} \rightarrow x, t\right)$ which is the probability density of reaching position $x$ after a time $t$ starting from $x_{0}$. When diffusion takes place in a homogeneous medium without boundaries, the propagator is a Gaussian distribution centered on $x_{0}$ with variance $2 D t$, where $D$ is the diffusion coefficient in the medium. On the other hand diffusion in complex systems such as biological cells or composite materials may exhibit non-Gaussian behavior due to confinement, hindrance by semi-permeable barriers or heterogeneity of the diffusion coefficient.

Generally speaking, the diffusion propagator obeys the diffusion equation:

$$
\frac{\partial G}{\partial t}=\nabla(D \nabla G), \quad G\left(x_{0} \rightarrow x, t=0\right)=\delta\left(x-x_{0}\right)
$$

where $\delta$ is the Dirac distribution, $\nabla=\frac{\partial}{\partial x}$ in the one-dimensional case, and the diffusion coefficient $D$ can in general be space and time dependent to capture heterogeneities of the medium $[1,2]$. Throughout this article, we refer to $\nabla(D \nabla)$ as the "diffusion operator". Note that if the diffusion coefficient is uniform, then the diffusion operator is simply proportional to the Laplace operator $\nabla^{2}$. The complexity of the geometry is hidden in the boundary conditions imposed on $G$ at the outer boundaries and possible inner semi-permeable barriers. Analytical solutions of Eq. (1) mainly rely on spectral decomposition over the diffusion operator eigenmodes which are explicitly known only for few geometries: slab, disk,

N. Moutal

PMC, CNRS Ecole Polytechnique, F-91128, Palaiseau, France

Tel: +33169334696

E-mail: nicolas.moutal@polytechnique.edu

D. Grebenkov

PMC, CNRS Ecole Polytechnique, F-91128, Palaiseau, France 
sphere (and some simple extensions) [3]. The study of more complicated structures requires numerical simulations such as stochastic Monte-Carlo simulations [4, 5] or PDE solving with finite element or finite difference methods [6]. On top of being time-consuming these techniques give little theoretical insight into the dependence of the propagator on the physical parameters of the simulated medium. In this situation, one-dimensional models of heterogeneous systems partitioned by semi-permeable barriers can help to uncover this dependence and to understand the role of diffusive exchange across the barriers. Note that three-dimensional diffusion in a stack of parallel planes with lateral invariance is naturally reduced to one-dimensional models. As a consequence, these models have a wide variety of applications, for example multilayer electrodes [7-9], coating of electronic components and improving the performance of semi-conductors [10-12], geophysics and thermal analyses of buildings [13-17], industrial processes [18-20], waste disposal and gas permeation in soils [21-24], drug delivery [25-27] and modeling tumor growth [28]. They can also be applied as approximation schemes for finding the spectrum of SturmLiouville problems where the coefficients of the differential operator are replaced by piecewise constant (or polynomial) functions (the so-called "Pruess method") [29-33]. Two applications of particular interest to us are diffusion magnetic resonance imaging (dMRI), a powerful experimental technique for probing diffusion inside complex media such as biological tissues (see Sec. 4.2), and first-passage phenomena (Sec. 4.3).

Because of this diversity of applications, many authors have more or less independently tackled such models of one-dimensional diffusion in heterogeneous structures, with various computational techniques: spectral decompositions, Green functions, Laplace transforms and others (see [34, 35] for a review of the subject). In this article we consider finite geometries, which are best treated by spectral decompositions (or "separation of variables"). To our knowledge, the most recent and complete work on this topic is the one by Hickson et al $[6,19,20]$. However it was mainly devoted to the case of heterogeneous structures with distinct diffusivities and without barriers. Moreover the spectrum was computed numerically and only few analytical results were obtained. On the other hand, some very general mathematical results were obtained by Gaveau et al for generic heterogeneous media without barriers [36]. Another technique was proposed in the recent work by Carr and Turner [37], in which the solution of Eq. (1) was decomposed on the Laplacian eigenmodes of each compartment separately, instead of the eigenmodes of the whole structure. This technique presents numerical advantages without providing analytical insights onto the spectrum of the diffusion operator.

In this article we present an efficient method to compute the eigenvalues and eigenfunctions of the diffusion operator in one-dimensional domains with multiple barriers. This method allows us to calculate the diffusion propagator and related quantities such as dMRI signal or first exit time distribution analytically for sufficiently regular geometries such as a finite periodic geometry or a micro-structure inside a larger scale structure, and numerically for arbitrary structures.

The article is organized as follows. Section 2 is entirely devoted to analytics. We start with standard computations using transition matrices (Sec. 2.1) and obtain the equation of the spectrum as a transcendental equation $F(\lambda)=0$ (Eq. (22)). Three following subsections are more technical and may be omitted in a first reading. In particular, we express the normalization constant of the eigenmodes as a function of $F$ (Eq. (24)), and we derive general consequences of the symmetry or the periodicity of the medium (Sec. 2.3 and 2.4, respectively). In Sec. 2.5, we study in more detail the function $F$ and obtain simple estimates of its roots with respect to the geometrical parameters of the medium, in particular the permeability of the barriers. This part is crucial for the numerical implementation of the method. This section is concluded with some extensions of our model. Section 3 illustrates our general approach on the example of a (finite) periodic structure with multiple identical barriers and compartments. The numerical implementation of the method is presented in Sec. 4.1. In particular, we discuss the major numerical challenges related to finding very close zeros of the eigenspectrum equation (22) and the proposed shortcuts based on the analytics from Sec. 2. The application of our technique to the computation of the dMRI signal and the first exit-time distribution is briefly discussed in Sec. 4.2 and 4.3. Section 5 concludes the paper and presents further perspectives and open problems.

The electronic Supplementary Material (SM) contains additional developments. Section SM. I is devoted to the application to dMRI. The dependence of the acquired signal on the geometrical parameters of the medium is thoroughly discussed. In Sec. SM. II, the effect of semi-permeable barriers on the diffusive 


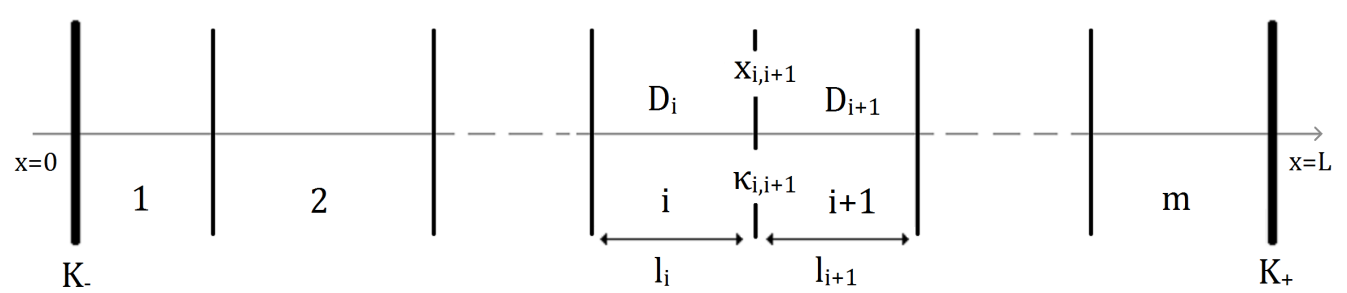

Fig. 1 Illustration of the geometry. Arbitrarily spaced barriers split the interval $[0, L]$ into $m$ compartments $\Omega_{i}$ of length $l_{i}$ and diffusion coefficient $D_{i}$. The positions of the barriers are denoted by $x_{i, i+1}$ and their permeabilities by $\kappa_{i, i+1}$. One can also take into account relaxation or leakage at the two outer barriers by permeabilities $K_{-}, K_{+}$.

motion is studied from another viewpoint, namely the first exit time distribution. Some technical results are moved to Sec. SM. III, which contains proofs of the existence of infinitely many eigenvalues, their non-degeneracy, their monotonic growth with respect to the barrier permeabilities, as well as a Courant nodal theorem for our particular model of diffusion with barriers.

\section{Computation of the eigenmodes of the diffusion operator}

\subsection{General case}

In this section we study the eigenmodes of the "diffusion operator" $\nabla(D \nabla)$ in a one-dimensional geometry (see Fig. 1). We reproduce the general computational scheme from Ref. [38] and propose improvements specific to the one-dimensional geometry. An interval $[0, L]$ is divided by barriers into $m$ compartments (or "cells") $\Omega_{i}=\left(x_{i-1, i}, x_{i, i+1}\right), i=1, \ldots, m$, where $x_{1,2}, \ldots, x_{m-1, m}$ are the positions of $m-1$ inner barriers, and $x_{0,1}=0$ and $x_{m, m+1}=L$ correspond to the outer barriers. Each compartment is characterized by its length $l_{i}=x_{i, i+1}-x_{i-1, i}>0$ and diffusion coefficient $D_{i}>0$ and each barrier by its permeability $\kappa_{i, i+1} \geq 0$ or equivalently by its "resistance" to diffusive exchange: $r_{i, i+1}=1 / \kappa_{i, i+1}$. Finally one can take into account some relaxation or leakage at the endpoints by non-negative permeabilities (or relaxaton coefficients) $K_{-}$and $K_{+}$.

The diffusion coefficient $D$ is thus a piecewise constant function:

$$
D(x)=\sum_{i=1}^{m} D_{i} I_{\Omega_{i}}(x)
$$

where $I_{\Omega_{i}}$ denotes the indicator function of $\Omega_{i}: I_{\Omega_{i}}(x)=1$ if $x \in \Omega_{i}$ and 0 otherwise. This implies that the diffusion operator can be split into two terms:

$$
\nabla(D \nabla)=D \nabla^{2}+(\nabla D) \nabla=D \nabla^{2}+\left(\sum_{i=1}^{m-1}\left(D_{i+1}-D_{i}\right) \delta\left(x-x_{i, i+1}\right)\right) \nabla .
$$

The second term vanishes at the interior points so that the diffusion operator is reduced to $D \nabla^{2}$. The same is true for the general class of diffusion operators $\nabla\left(D^{\alpha} \nabla\left(D^{1-\alpha} \cdot\right)\right)$, where $0 \leq \alpha \leq 1$ is the ItôStratonovitch interpretation parameter (some authors use $1-\alpha$ instead of $\alpha$ ) [39, 40]. Here we consider heterogeneous diffusion coefficients with discontinuities at the barriers, hence these operators coincide inside the compartments but yield different boundary conditions at the barriers. Our choice $\nabla(D \nabla)$ corresponds to the Hänggi-Klimontovich interpretation [41-45] with $\alpha=1$, which is most often used in physical applications. The main reason is that it corresponds to the standard Fick law and that equilibrium solutions of the diffusion equation are constant, which is expected for, say, water diffusing in an isothermal medium. From a mathematical point of view, this choice ensures that the operator is self-adjoint, which allows us to use standard spectral methods. 
The $L^{2}$-normalized eigenmodes $u$ of the diffusion operator are then determined by the equation

$$
D u^{\prime \prime}+\lambda u=0,
$$

with the boundary conditions

$$
\begin{array}{ll}
\left.D_{i} u^{\prime}\right|_{\Omega_{i}}=\left.D_{i+1} u^{\prime}\right|_{\Omega_{i+1}} & \text { at the barrier at } x_{i, i+1} \\
\left.D_{i} u^{\prime}\right|_{\Omega_{i}}=\kappa_{i, i+1}\left(\left.u\right|_{\Omega_{i+1}}-\left.u\right|_{\Omega_{i}}\right) & \text { at the barrier at } x_{i, i+1} \\
D_{1} u^{\prime}(0)=K_{-} u(0) & \\
D_{m} u^{\prime}(L)=-K_{+} u(L), &
\end{array}
$$

and the normalization condition

$$
\int_{0}^{L} u^{2}=1
$$

where $\left.u\right|_{\Omega_{i}}$ is the restriction of $u$ to the cell $\Omega_{i}(i=1, \ldots, m)$ and prime denotes the derivative with respect to $x$.

Eqs. (5) and (6) express the flux conservation across the barriers (no accumulation of diffusing particles) and the drop of particle density due to the non-zero resistance of the barriers, respectively. Note in particular that Eq. (5) ensures the continuity of $D \nabla u=D u^{\prime}$. The infinitely thin barriers that we consider can approximate barriers of thickness $h_{i, i+1}$ with the standard continuity conditions. When $h_{i, i+1}$ is much smaller than other length scales, one can interpret $\kappa_{i, i+1} h_{i, i+1}$ as the diffusion coefficient inside the barrier, whereas $\left(\left.u\right|_{\Omega_{i+1}}-\left.u\right|_{\Omega_{i}}\right) / h_{i, i+1}$ approximates the derivative of $u$ across the barrier of thickness $h_{i, i+1}$. If $\kappa_{i, i+1}=\infty$ there is no barrier and Eq. (6) becomes a continuity condition for $u$ at $x=x_{i, i+1}$. In the opposite limit $\kappa_{i, i+1}=0$ the compartments $\Omega_{i}$ and $\Omega_{i+1}$ do not communicate with each other: the flux $D u^{\prime}$ is zero at the barrier and the discontinuity $\left(\left.u\right|_{\Omega_{i+1}}-\left.u\right|_{\Omega_{i}}\right)\left(x_{i, i+1}\right)$ is arbitrary. One can then study the two parts $\left[0, x_{i, i+1}\right]$ and $\left[x_{i, i+1}, L\right]$ separately.

To avoid such trivial separations, we consider only non-zero permeabilities: $\kappa_{i, i+1}>0$ throughout this article. Under this assumption we prove in Sec. SM. III that there are infinitely many eigenvalues $\lambda_{n}, n=1,2, \ldots$, and all $\lambda_{n}$ are simple. One can also easily prove that they are non-negative, and we sort them by ascending order: $0 \leq \lambda_{1}<\lambda_{2}<\ldots$. Moreover, thanks to the self-adjointness of the diffusion operator $\nabla(D \nabla)$ we know that the eigenmodes $u_{n}, n=1,2, \ldots$ form a complete orthonormal basis in the space $L^{2}(0, L)$ of square-integrable functions on $(0, L)[34,35]$.

For simplicity we further assume that $K_{-}<\infty$, which allows us to write

$$
u=\beta v, \quad v(0)=1,
$$

with $\beta$ being a normalization constant that ensures Eq. (9). The case of Dirichlet boundary conditions $\left(K_{-}=\infty\right)$ requires another convention which is detailed in Sec. SM. IV.5. We study the (non-normalized) eigenmode $v$ first and then we compute the normalization constant $\beta$.

Throughout this section we assume $\lambda \neq 0$. One can see that $\lambda=0$ is only possible if the relaxation coefficients $K_{ \pm}$are equal to zero and in this case one gets a constant eigenmode $v=1$ (and $\beta=1 / \sqrt{L}$ ).

Equation (4) has a general solution

$$
\left.v\right|_{\Omega_{i}}(x)=a_{i}^{l} \cos \left(\sqrt{\lambda / D_{i}}\left(x-x_{i-1, i}\right)\right)+b_{i}^{l} \sin \left(\sqrt{\lambda / D_{i}}\left(x-x_{i-1, i}\right)\right),
$$

or equivalently

$$
\left.v\right|_{\Omega_{i}}(x)=a_{i}^{r} \cos \left(\sqrt{\lambda / D_{i}}\left(x-x_{i, i+1}\right)\right)+b_{i}^{r} \sin \left(\sqrt{\lambda / D_{i}}\left(x-x_{i, i+1}\right)\right),
$$

where $a_{i}^{l}, b_{i}^{l}$ and $a_{i}^{r}, b_{i}^{r}$ are constants to be determined, related by

$$
\left[\begin{array}{l}
a_{i}^{r} \\
b_{i}^{r}
\end{array}\right]=\mathcal{R}_{i}\left[\begin{array}{l}
a_{i}^{l} \\
b_{i}^{l}
\end{array}\right], \quad \text { where } \quad \mathcal{R}_{i}=\left[\begin{array}{cc}
\cos \left(\sqrt{\lambda / D_{i}} l_{i}\right) & \sin \left(\sqrt{\lambda / D_{i}} l_{i}\right) \\
-\sin \left(\sqrt{\lambda / D_{i}} l_{i}\right) & \cos \left(\sqrt{\lambda / D_{i}} l_{i}\right)
\end{array}\right] .
$$


Note that

$$
\left.v\right|_{\Omega_{i}}\left(x_{i, i+1}\right)=a_{i}^{r},\left.\quad D_{i} v^{\prime}\right|_{\Omega_{i}}\left(x_{i, i+1}\right)=\sqrt{\lambda D_{i}} b_{i}^{r}
$$

with similar formulas for $a_{i}^{l}, b_{i}^{l}$, so that one can write the boundary equations (5) and (6) as

$$
\left[\begin{array}{l}
a_{i+1}^{l} \\
b_{i+1}^{l}
\end{array}\right]=\mathcal{K}_{i, i+1}\left[\begin{array}{l}
a_{i}^{r} \\
b_{i}^{r}
\end{array}\right], \quad \text { with } \quad \mathcal{K}_{i, i+1}=\left[\begin{array}{ll}
1 & r_{i, i+1} \sqrt{\lambda D_{i}} \\
0 & \sqrt{D_{i} / D_{i+1}}
\end{array}\right]
$$

The equations at the barriers can thus be restated in a matrix form:

$$
\left[\begin{array}{l}
a_{i+1}^{l} \\
b_{i+1}^{l}
\end{array}\right]=\mathcal{M}_{i, i+1}\left[\begin{array}{l}
a_{i}^{l} \\
b_{i}^{l}
\end{array}\right]
$$

with the notation for the "transition matrix":

$$
\mathcal{M}_{i, i+1}=\mathcal{K}_{i, i+1} \mathcal{R}_{i}
$$

with $\mathcal{R}_{i}$ and $\mathcal{K}_{i, i+1}$ defined by Eqs. (13), (15). In the same way, one can rewrite the endpoint conditions $(7),(8)$ :

$$
\left[-K_{-} \sqrt{\lambda D_{1}}\right]\left[\begin{array}{c}
a_{1}^{l} \\
b_{1}^{l}
\end{array}\right]=0 \quad \text { and } \quad\left[K_{+} \sqrt{\lambda D_{m}}\right]\left[\begin{array}{l}
a_{m}^{r} \\
b_{m}^{r}
\end{array}\right]=0
$$

We have the additional condition $a_{1}^{l}=v(0)=1$, therefore

$$
\left[\begin{array}{l}
a_{1}^{l} \\
b_{1}^{l}
\end{array}\right]=\left[\begin{array}{c}
1 \\
K_{-} / \sqrt{\lambda D_{1}}
\end{array}\right] \quad \text { and } \quad\left[\begin{array}{l}
a_{m}^{r} \\
b_{m}^{r}
\end{array}\right]=\epsilon\left[\begin{array}{c}
1 \\
-K_{+} / \sqrt{\lambda D_{m}}
\end{array}\right]
$$

where $\epsilon$ is an unknown proportionality coefficient.

Equation (16), which relates the coefficients of one cell to those of the next cell, is compatible with Eq. (18), which prescribes the first and last cell coefficients (up to a proportionality factor), only if $\lambda$ is an actual eigenvalue of the diffusion operator $\nabla(D \nabla)$. That is, by writing explicitly the condition that the product of all the transition matrices $\mathcal{M}_{i, i+1}$ should send the previously determined $\left(a_{1}^{l}, b_{1}^{l}\right)$ onto the $\left(a_{m}^{l}, b_{m}^{l}\right)$, we get the equation on the spectrum of the diffusion operator:

$$
\mathcal{T}\left[\begin{array}{c}
1 \\
K_{-} / \sqrt{\lambda D_{1}}
\end{array}\right]=\epsilon\left[\begin{array}{c}
1 \\
-K_{+} / \sqrt{\lambda D_{m}}
\end{array}\right]
$$

with

$$
\mathcal{T}=\mathcal{R}_{m} \mathcal{M}_{m-1, m} \ldots \mathcal{M}_{1,2}
$$

Note that this condition is equivalent to

$$
\left[K_{+} / \sqrt{\lambda D_{m}} \quad 1\right] \mathcal{T}=\eta\left[-K_{-} / \sqrt{\lambda D_{1}} \quad 1\right]
$$

and to

$$
F(\lambda):=\left[K_{+} / \sqrt{\lambda D_{m}} \quad 1\right] \mathcal{T}(\lambda)\left[\begin{array}{c}
1 \\
K_{-} / \sqrt{\lambda D_{1}}
\end{array}\right]=0
$$

The proportionality coefficients $\epsilon$ and $\eta$ are constrained by the relation: $\epsilon \eta=\operatorname{det} \mathcal{T}=\sqrt{\frac{D_{1}}{D_{m}}}$. 


\subsection{Computation of the norm}

Now we compute the normalization constant $\beta$. Since the eigenmode $v$ is a piecewise combination of sine and cosine functions, the constant $\beta$ can be obtained by a direct integration (see Ref. [38]). This approach is convenient for numerical computations. Here we present another approach which is more suitable for analytical derivations. The starting point of the method is the spectral decomposition of the diffusion propagator:

$$
G\left(t, x_{0} \rightarrow x\right)=\sum_{n=1}^{\infty} u_{n}\left(x_{0}\right) u_{n}(x) e^{-\lambda_{n} t}=\sum_{n=1}^{\infty} \beta_{n}^{2} v_{n}\left(x_{0}\right) v_{n}(x) e^{-\lambda_{n} t},
$$

where $n=1,2, \ldots$ spans the infinitely many eigenmodes of the diffusion operator. We now compute this propagator in a different way by solving explicitly Eq. (1). Again, we use Eq. (3) to transform $\nabla(D \nabla)$ into $D \nabla^{2}$ at the interior points. Let $\tilde{G}\left(s, x_{0} \rightarrow x\right)$ denote the Laplace transform of the propagator: $\tilde{G}\left(s, x_{0} \rightarrow x\right)=\int_{0}^{\infty} e^{-s t} G\left(t, x_{0} \rightarrow x\right) \mathrm{d} t$. Then $\tilde{G}$ obeys the equation

$$
D(x) \tilde{G}^{\prime \prime}\left(s, x_{0} \rightarrow x\right)=s \tilde{G}\left(s, x_{0} \rightarrow x\right)-\delta\left(x-x_{0}\right),
$$

with the same boundary conditions (5)-(8) as for the propagator $G$ in time domain. As in the previous section, prime denotes derivative with respect to $x$. We use the method from Sec. 2.1 to solve the homogeneous equation with the inner boundary conditions (5), (6) imposed at the barriers: if $s \neq 0$ we can build two solutions $\phi(s, x)$ and $\psi(s, x)$ such that:

$-\phi(s, x)$ is built from $\left[\begin{array}{l}a_{1}^{l} \\ b_{1}^{l}\end{array}\right]=\left[\begin{array}{l}1 \\ 0\end{array}\right]$ : at the left endpoint its derivative with respect to $x$ is zero and its value is one.

$-\psi(s, x)$ is built from $\left[\begin{array}{l}a_{1}^{l} \\ b_{1}^{l}\end{array}\right]=\left[\begin{array}{l}0 \\ 1\end{array}\right]$ : at the left endpoint its derivative with respect to $x$ is $\sqrt{s / D_{1}}$ and its value is zero.

It is then easy to obtain the complete solution because the Wronskian matrix $\mathcal{W}=\left[\begin{array}{cc}\phi(s, x) & \psi(s, x) \\ \phi^{\prime}(s, x) & \psi^{\prime}(s, x)\end{array}\right]$ is quite simple. Indeed over any layer $\Omega_{i}$ the determinant of $\mathcal{W}$ is constant and equal to $\sqrt{s D_{1}} / D_{i}$. This is obtained from the differential equation obeyed by $\phi(s, x)$ and $\psi(s, x)$ and the boundary conditions at each barrier. The standard method for solving the second order differential equations then yields

$$
\tilde{G}=\mu \phi+\nu \psi
$$

with the equation on $\mu, \nu$ :

$$
D(x)\left[\begin{array}{c}
\mu^{\prime}(s, x) \\
\nu^{\prime}(s, x)
\end{array}\right]=\mathcal{W}^{-1}\left[\begin{array}{c}
0 \\
-\delta\left(x-x_{0}\right)
\end{array}\right]=-\frac{D(x)}{\sqrt{D_{1} s}} \delta\left(x-x_{0}\right)\left[\begin{array}{c}
-\psi(s, x) \\
\phi(s, x)
\end{array}\right] .
$$

After a straightforward integration, we obtain

$$
\begin{aligned}
\tilde{G}\left(x_{0} \rightarrow x, s\right) & =\left(A+\frac{1}{\sqrt{D_{1} s}} \psi\left(s, x_{0}\right) H\left(x-x_{0}\right)\right) \phi(s, x) \\
& +\left(B-\frac{1}{\sqrt{D_{1} s}} \phi\left(s, x_{0}\right) H\left(x-x_{0}\right)\right) \psi(s, x),
\end{aligned}
$$

which is valid for any $x_{0}, x \in[0, L]$, and $s \neq 0$, where $H$ is the Heaviside function and the constants $A$ and $B$ remain to be determined. We consider general relaxing conditions at the endpoints:

$$
\left\{\begin{array}{l}
D_{1} \frac{\partial \tilde{G}}{\partial x}(x=0)=K_{-} \tilde{G}(x=0) \\
D_{m} \frac{\partial \tilde{G}}{\partial x}(x=L)=-K_{+} \tilde{G}(x=L)
\end{array},\right.
$$


from which

$$
\begin{aligned}
A & =\frac{\phi\left(s, x_{0}\right)\left(D_{m} \psi^{\prime}(s, L)+K_{+} \psi(s, L)\right)-\psi\left(s, x_{0}\right)\left(D_{m} \phi^{\prime}(s, L)+K_{+} \phi(s, L)\right)}{D_{m} K_{-} \psi^{\prime}(s, L)+K_{+} K_{-} \psi(s, L)+D_{m} \sqrt{D_{1} s} \phi^{\prime}(s, L)+K_{+} \sqrt{D_{1} s} \phi(s, L)} \\
B & =\frac{K_{-} A}{\sqrt{D_{1} s}}
\end{aligned}
$$

Now we simplify the above expressions. We anticipate that the non-normalized eigenmodes are $v_{n}(x)=$ $v\left(\lambda_{n}, x\right)$, with

$$
v(s, x)=\phi(s, x)+\frac{K_{-}}{\sqrt{D_{1} s}} \psi(s, x)
$$

and we use Eq. (14) to get

$$
A \phi(s, x)+B \psi(s, x)=\frac{v(s, x) \phi\left(s, x_{0}\right)}{K_{-}}-\frac{\sqrt{D_{1} s}}{K_{-}} v(s, x) v\left(s, x_{0}\right) \frac{\left[K_{+} \sqrt{D_{m} s}\right] \mathcal{T}(s)\left[\begin{array}{l}
1 \\
0
\end{array}\right]}{F(s)}
$$

with $\mathcal{T}$ and $F$ defined in Eqs. (20), (22), respectively, in which $\lambda$ is replaced by $s$. To obtain the propagator in time domain, one needs to perform an inverse Laplace transform. This is done by looking for the poles $s=\lambda_{n}$ of $\tilde{G}$ and the above formula shows that they are given by the zeros of $F(s)$, as expected. We prove in Sec. SM. III.2 that these zeros are simple. At $s=\lambda_{n}$, one can use Eqs. (19) and (21) to compute the residue of $\tilde{G}$, which yields simply

$$
\operatorname{Res}_{s=\lambda_{n}}(\tilde{G})=\left.\frac{-\eta_{n} \sqrt{D_{1} s} v(s, x) v\left(s, x_{0}\right)}{\frac{\mathrm{d} F}{\mathrm{~d} s}}\right|_{s=\lambda_{n}}
$$

By comparison with Eq. (23), this allows us to conclude:

$$
\beta_{n}^{-2}=-\frac{1}{\eta_{n} \sqrt{D_{1} \lambda_{n}}} \frac{\mathrm{d} F}{\mathrm{~d} \lambda}\left(\lambda_{n}\right)
$$

In general, one obtains $\eta_{n}$ by computing the matrix product in Eq. (21). A great simplification occurs in the case of symmetric geometries, which is the topic of the next section.

\subsection{Symmetry properties}

For a geometry which is symmetric with respect to the middle of the interval $[0, L]$, some simplifications occur. In fact the symmetry of the geometry implies that the eigenmodes are either symmetric or antisymmetric with respect to the middle of the interval, and as a consequence $\epsilon=\eta=+1$ or $\epsilon=\eta=$ -1 , respectively. These statements can be easily proved with the above matrix formalism. In fact, the symmetry of the geometry is equivalent to the two properties:

1. The endpoints vectors $\mathcal{V}_{+}=\left[\begin{array}{c}1 \\ -K_{+} / \sqrt{\lambda D_{1}}\end{array}\right]$ and $\mathcal{V}_{-}=\left[\begin{array}{c}1 \\ K_{-} / \sqrt{\lambda D_{m}}\end{array}\right]$ have equal first components and opposite second components, which follows from the symmetry $K_{-}=K_{+}, D_{1}=D_{m}$. With the notation $\mathcal{S}=\left[\begin{array}{cc}1 & 0 \\ 0 & -1\end{array}\right]$, this can be restated as $\mathcal{V}_{ \pm}=\mathcal{S} \mathcal{V}_{\mp}$

2. The inverse of the transition matrix $\mathcal{T}$ is obtained by replacing the off-diagonal terms by their opposite in its expression (note that this corresponds to the transformation $\sqrt{\lambda} \rightarrow-\sqrt{\lambda}$ ). In fact, this property is clearly true for the "elementary blocks" $\mathcal{K}$ and $\mathcal{R}$ and thus it is also the case for $\mathcal{R}_{m} \mathcal{K}_{m-1, m} \mathcal{R}_{m-1} \ldots \mathcal{K}_{1,2} \mathcal{R}_{1}$ because $\mathcal{R}_{i}=\mathcal{R}_{m+1-i}$ and $\mathcal{K}_{i, i+1}=\mathcal{K}_{m-i, m+1-i}$. In other words, $\mathcal{T}^{-1}=\mathcal{S} \mathcal{T} \mathcal{S}$. 
The consequence of these two properties is that Eq. (19) can be restated as: " $\mathcal{V}_{-}$is an eigenvector of $\mathcal{S} \mathcal{T}$ " and that this matrix is equal to its inverse:

$$
(\mathcal{S T})^{-1}=\mathcal{T}^{-1} \mathcal{S}^{-1}=\mathcal{S T}
$$

This implies that the eigenvalues of this matrix, hence the proportionality coefficients $\epsilon, \eta$ in Eqs. (19) and $(21)$, are equal to \pm 1 . We can also easily prove the symmetry or anti-symmetry of the eigenmodes. In fact, one has

$$
\begin{aligned}
{\left[\begin{array}{l}
a_{i}^{l} \\
b_{i}^{l}
\end{array}\right] } & =\mathcal{K}_{i-1, i} \mathcal{R}_{i-1} \ldots \mathcal{R}_{1} \mathcal{V}_{-} \\
{\left[\begin{array}{c}
a_{m+1-i}^{r} \\
b_{m+1-i}^{r}
\end{array}\right] } & =\mathcal{K}_{m+1-i, m+2-k}^{-1} \mathcal{R}_{m+2-k}^{-1} \ldots \mathcal{R}_{m}^{-1} \epsilon \mathcal{V}_{+}
\end{aligned}
$$

Hence

$$
\left[\begin{array}{l}
a_{m+1-i}^{r} \\
b_{m+1-i}^{r}
\end{array}\right]=\mathcal{S} \mathcal{K}_{i-1, i} \mathcal{S} \mathcal{S} \mathcal{R}_{i-1} \mathcal{S} \ldots \mathcal{S} \mathcal{R}_{1} \mathcal{S} \in \mathcal{V}_{+}=\epsilon \mathcal{S}\left[\begin{array}{c}
a_{i}^{l} \\
b_{i}^{l}
\end{array}\right]
$$

Let $x \in \Omega_{i}$, we write $x=x_{i-1, i}+\xi$, with $0<\xi<l_{i}$, which implies by symmetry that $L-x=$ $x_{m+1-i, m+2-i}-\xi$. According to Eqs. (11), (12), and (25), we have then

$$
\begin{aligned}
v(x) & =\left[\begin{array}{ll}
a_{i}^{l} & b_{i}^{l}
\end{array}\right]\left[\begin{array}{l}
\cos \left(\xi \sqrt{\lambda / D_{i}}\right) \\
\sin \left(\xi \sqrt{\lambda / D_{i}}\right)
\end{array}\right] \\
& =\epsilon\left[a_{m+1-i}^{r} b_{m+1-i}^{r}\right]\left[\begin{array}{l}
\cos \left(-\xi \sqrt{\lambda / D_{m+1-i}}\right) \\
\sin \left(-\xi \sqrt{\lambda / D_{m+1-i}}\right)
\end{array}\right]=\epsilon v(L-x),
\end{aligned}
$$

since $D_{i}=D_{m+1-i}$. Therefore the eigenmode is symmetric if $\epsilon=+1$ and anti-symmetric if $\epsilon=-1$. Moreover from Eq. (24) we deduce that the derivative $\frac{\mathrm{d} F}{\mathrm{~d} \lambda}\left(\lambda_{n}\right)$ and $\eta_{n}$ have opposite signs. Because the eigenvalues $\lambda_{n}$ are the zeros of $F$, the derivative alternates between positive and negative sign, and so do $\eta_{n}$ and $\epsilon_{n}$. In particular, in the case of a symmetric geometry, the modes $u_{n}$ are alternately symmetric and anti-symmetric. One can show that the first mode $u_{1}$ is always symmetric $\left(\epsilon_{1}=\eta_{1}=1\right)$, hence

$$
\epsilon_{n}=\eta_{n}=(-1)^{n-1} \text {. }
$$

\subsection{Periodicity properties}

A finite periodic geometry is an $M$-times repetition of an elementary block composed of $N$ compartments: $\left(D_{1} ; l_{1}\right),\left(D_{2} ; l_{2}\right), \ldots,\left(D_{N} ; l_{N}\right)$. The transition matrix of the block is

$$
\mathcal{M}=\mathcal{K}_{\text {inter }} \mathcal{R}_{N} \mathcal{K}_{N-1, N} \ldots \mathcal{R}_{1}
$$

where $\mathcal{K}_{\text {inter }}$ is the matrix corresponding to the inter-block barriers. Then the complete transition matrix $\mathcal{T}$ is equal to

$$
\mathcal{T}=\mathcal{K}_{\text {inter }}^{-1} \mathcal{M}^{M}
$$

Because of the periodicity,

$$
\operatorname{det} \mathcal{M}=\underbrace{\sqrt{\frac{D_{N}}{D_{1}}}}_{\operatorname{det} \mathcal{K}_{\text {inter }}} \sqrt{\frac{D_{N-1}}{D_{N}}} \cdots \sqrt{\frac{D_{1}}{D_{2}}}=1 .
$$

This property makes the computation of $\mathcal{M}^{M}$ easier, thanks to the formula

$$
\mathcal{M}^{M}=\frac{\sin M \psi}{\sin \psi} \mathcal{M}-\frac{\sin (M-1) \psi}{\sin \psi} \mathcal{I}_{2},
$$




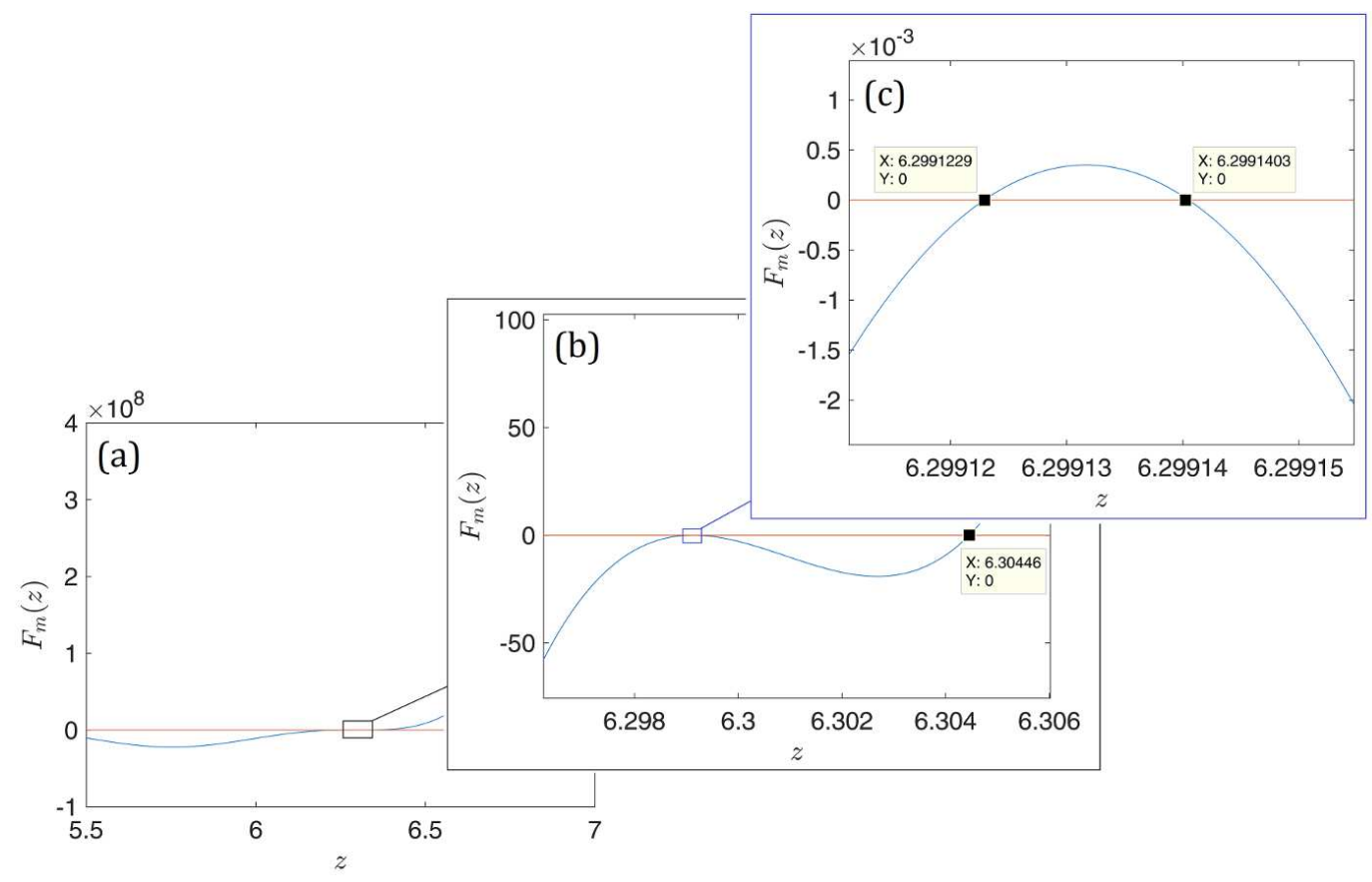

Fig. 2 Example of roots which may prove challenging to find numerically with standard methods. We consider five compartments and $D_{1}=\ldots=D_{5}=1, r_{1,2}=\ldots=r_{4,5}=10$ and the lengths $l_{i}$ of the five compartments are: $1 ; 1.2 ; 1.5 ; 1.2 ; 1$, with reflecting boundary conditions at the endpoints: $K_{ \pm}=0$. The root $z=6.30446$ (b) corresponds to $l_{3}=1.5$, with $n=3, \zeta=2$, whereas the two roots $z_{ \pm}=6.2991316 \pm 8.7 \cdot 10^{-6}$ (c) correspond to $l_{1}=l_{5}=1$, with $n=2$, $\zeta=1$ (see explanations in the text). Notice the scale changes, horizontally and vertically, between (a), (b) and (c).

where $\mathcal{I}_{2}$ is the $2 \times 2$ identity matrix and $\psi$ is implicitly defined by

$$
\cos \psi=\frac{1}{2} \operatorname{Tr} \mathcal{M} .
$$

Formula (29) implies that the inter-block variation of the coefficients $a, b$ has the form:

$$
a_{i_{0}+N(j-1)}=A \cos (j \psi)+B \sin (j \psi), \quad j=1, \ldots, M,
$$

with a similar formula for $b$, where $A$ and $B$ are coefficients which depend on the choice of the origin $i_{0} \in\{1, \ldots, N-1\}$. Thus $\psi$ governs the global behavior of the mode (when the number $M$ of repeated blocks is sufficiently large).

\subsection{Study of the spectrum}

The main numerical difficulty of the above method is to solve Eq. (22) on the spectrum, that is to find the zeros of $F(\lambda)$. In fact, a standard method to find all the zeros of a function in a given interval is to compute the function on a fine array $(0, \epsilon, 2 \epsilon, \ldots)$ and to look for the sign changes, that indicate the presence of at least one zero. By decreasing $\epsilon$, one is assured at some point to find all the zeros of the function. However, in general one knows neither the number of zeros of the function in a given interval nor the minimal spacing between the zeros. In turn, missing some zeros would result in missed eigenmodes, and thus in inaccurate computation of the propagator and the related diffusion quantities. An example of $F(\lambda)$ shown in Fig. 2 illustrates that some roots may be very close to each other. We provide here a rough analysis of Eq. (22) in order to study this phenomenon.

We discard the elementary case of a single interval $(m=1)$ where the roots of $F$ are explicitly known $[1,2]$. Let us assume for simplicity that all the diffusion coefficients $D_{i}$ and the barrier resistances $r_{i, i+1}$ 
are identical (denoted $D$ and $r$, respectively). Furthermore we set the relaxation coefficients $K_{ \pm}$to zero. We change the variable $\lambda$ by $z=\sqrt{\lambda / D}$ and reveal an explicit dependence of $F$ on the geometry (omitting $D$ and $r$ for the sake of clarity):

$$
F(\lambda)=F_{m}\left(z ; l_{1}, \ldots, l_{m}\right)
$$

\subsubsection{Regime $r \rightarrow 0$}

First we consider the regime of quasi-permeable barriers, that is $r \rightarrow 0$. One has

$$
\mathcal{K}=\mathcal{I}_{2}+r D z \mathcal{N}, \quad \mathcal{N}=\left[\begin{array}{ll}
0 & 1 \\
0 & 0
\end{array}\right],
$$

from which we deduce the first-order expansion

$$
F_{m}\left(z ; l_{1}, \ldots, l_{m}\right) \approx-\sin (z L)+r D z \sum_{i=1}^{m} \sin \left(z\left(l_{1}+\ldots+l_{i}\right)\right) \sin \left(z\left(l_{i+1}+\ldots+l_{m}\right)\right) .
$$

This formula implies that the roots are approximately equal to $z_{0}=n \pi / L$, with an integer $n$. In fact, one can compute the first order correction to this formula, which yields

$$
z \approx \frac{n \pi}{L}\left(1-\frac{r D}{l}\left[\frac{1}{m} \sum_{i=1}^{m} \sin ^{2}\left(n \pi \frac{l_{1}+\cdots+l_{i}}{L}\right)\right]\right)
$$

where $l=L / m$ is the arithmetic mean of the $l_{i}$. The factor inside the brackets is always less than 1 , hence the (first order) relative perturbation of the roots is at most $r D / l$. Therefore in the regime of quasi-permeable inner barriers $(r D / l \ll 1)$ the roots are easy to find numerically because we have a good estimate of their position and a good lower bound of the distance between them.

\subsubsection{Regime $r \rightarrow \infty$}

Now we turn to the opposite regime of almost impermeable barriers: $r \rightarrow \infty$. In this case one writes

$$
\mathcal{K}=r D z\left(\mathcal{N}+\frac{1}{r D z} \mathcal{I}_{2}\right)
$$

For $z$ large enough such that $r D z \gg 1$, this yields

$$
F_{m}\left(z ; l_{1}, \ldots, l_{m}\right) \approx(-r D z)^{m-1} \sin \left(z l_{1}\right) \cdots \sin \left(z l_{m}\right)\left(1-\frac{1}{r D z} \sum_{i=1}^{m-1} \frac{\sin \left(z\left(l_{i}+l_{i+1}\right)\right)}{\sin \left(z l_{i}\right) \sin \left(z l_{i+1}\right)}+\ldots\right) .
$$

From this expression one gets the approximate roots $z_{0}=n \pi / l_{i}$ with an integer $n$, as expected. The nonzero permeability of the barriers increases the values of the roots by coupling the compartments to their nearest neighbors. The higher-order terms of the expansion (33) involve coupling between next-nearest neighbors, etc. From the above formula we expect the increase to be of order $\left(r D z_{0}\right)^{-1}$. The case $n=0$ (that is, $z_{0}=0$ ) is special and we treat it later. Note that the above expansion is valid around $z_{0}=n \pi / l_{i}$ (with $n>0$ ) if $r D z_{0} \gg 1$, that is $r D / l_{i} \gg 1$.

If we consider an isolated root $z_{0}=n \pi / l_{i}$ (which means that all the other $n^{\prime} \pi / l_{i^{\prime}}$ are located at a relative distance much greater that $\left.\left(r D z_{0}\right)^{-1}\right)$, then we get

$$
z \approx \frac{n \pi}{l_{i}}+\frac{\zeta_{i}}{n \pi r D}
$$

where $\zeta_{i}$ is the number of neighbors of the cell $i\left(\zeta_{i}=2\right.$ if $1<i<m$, otherwise $\left.\zeta_{i}=1\right)$.

The case of non-isolated roots is more complicated but also more interesting. In fact all the numerical difficulties come from this case. From the equation

$$
\mathcal{R}_{i}\left[\begin{array}{l}
1 \\
0
\end{array}\right]\left[\begin{array}{ll}
0 & 1
\end{array}\right] \mathcal{R}_{i}-\left[\begin{array}{l}
1 \\
0
\end{array}\right]\left[\begin{array}{ll}
0 & 1
\end{array}\right]=-\sin \left(z l_{i}\right) \mathcal{R}_{i}
$$


we deduce the following general relation which is valid for any $i$ from 1 to $m-1$ :

$$
F_{m}\left(z ; l_{1}, \ldots, l_{m}\right)=\frac{\left[F_{i}\left(z ; l_{1}, \ldots, l_{i}\right) F_{m+1-i}\left(z ; l_{i}, \ldots, l_{m}\right)-F_{i-1}\left(z ; l_{1}, \ldots, l_{i-1}\right) F_{m-i}\left(z ; l_{i+1}, \ldots, l_{m}\right)\right]}{F_{1}\left(z ; l_{i}\right)} .
$$

Now we assume that there exist $i_{1}<i_{2}$ such that

$$
z_{0}=\frac{n_{1} \pi}{l_{i_{1}}}=\frac{n_{2} \pi}{l_{i_{2}}},
$$

with $n_{1}, n_{2}$ integers. Note that $n_{1} / n_{2}=l_{i_{1}} / l_{i_{2}}$. We look for an approximate root of the form $z=z_{0}(1+\eta)$, with $\eta \sim\left(r D z_{0}\right)^{-1}$ (where $\sim$ means "is of the same order of magnitude as").

First let us consider the case where two compartments $i_{1}$ and $i_{2}$ are not neighbors, that is $i_{1}+1<i_{2}$. From Eq. (33) we infer

$$
\begin{aligned}
& F_{i_{1}+1}\left(z ; l_{1}, \ldots, l_{i_{1}+1}\right) \sim(r D z)^{i_{1}} \eta \sim(r D z)^{i_{1}-1} \\
& F_{m-i_{1}}\left(z ; l_{i_{1}+1}, \ldots, l_{m}\right) \sim(r D z)^{m-1-i_{1}} \eta \sim(r D z)^{m-2-i_{1}} \\
& F_{i_{1}}\left(z ; l_{1}, \ldots, l_{i_{1}}\right) \sim(r D z)^{i_{1}-1} \eta \sim(r D z)^{i_{1}-2} \\
& F_{m-1-i_{1}}\left(z ; l_{i_{1}+2}, \ldots, l_{m}\right) \sim(r D z)^{m-2-i_{1}} \eta \sim(r D z)^{m-3-i_{1}}
\end{aligned}
$$

hence Eq. (35) becomes

$$
F_{m}\left(z ; l_{1}, \ldots, l_{m}\right)=\frac{F_{i_{1}}\left(z ; l_{1}, \ldots, l_{i_{1}}\right) F_{m+1-i_{1}}\left(z ; l_{i_{1}}, \ldots, l_{m}\right)}{F_{1}\left(z ; l_{i_{1}}\right)}\left(1+O\left((r D z)^{-2}\right)\right)
$$

We deduce that the roots of $F_{m}\left(z ; l_{1}, \ldots, l_{m}\right)$ are given by the roots of the functions $F_{i_{1}}\left(z ; l_{1}, \ldots, l_{i_{1}}\right)$ and $F_{m+1-i_{1}}\left(z ; l_{i_{1}}, \ldots, l_{m}\right)$, which are not coupled to the first order in $(r D z)^{-1}$ :

$$
z \approx z_{0}+\frac{\zeta_{i_{1}}}{n_{1} \pi r D} \quad \text { and } \quad z \approx z_{0}+\frac{\zeta_{i_{2}}}{n_{2} \pi r D}
$$

Note that the same is true for any number of "coinciding" roots as long as they correspond to nonadjacent compartments. The roots are at a relative distance of order $\left(r D z_{0}\right)^{-1}$ if $n_{1} / \zeta_{i_{1}} \neq n_{2} / \zeta_{i_{2}}$. If $n_{1} / \zeta_{i_{1}}=n_{2} / \zeta_{i_{2}}$ one has to compute the next-order corrections which involve the length of the other compartments, as explained previously. One can show that the term of order $\left(r D z_{0}\right)^{i_{1}-i_{2}}$ is always nonzero; for symmetric geometries $\left(r D z_{0}\right)^{i_{1}-i_{2}}$ may be the first non-zero term of the expansion of the relative difference of the roots.

Now we consider the case $i_{2}=i_{1}+1$. We use Eq. (33) to get

$$
F_{m}\left(z ; l_{1}, \ldots, l_{m}\right) \approx(-r D z)^{m-3}\left(\prod_{i \neq i_{1}, i_{1}+1} \sin \left(z l_{i}\right)\right)\left(n_{1} n_{2} X^{2}-\left(\zeta_{i_{1}} n_{1}+\zeta_{i_{2}} n_{2}\right) X+\left(\zeta_{i_{1}} \zeta_{i_{2}}-1\right)\right)
$$

where $X=r D \pi \eta$. Thus we obtain two roots:

$$
z_{ \pm}=z_{0}+\frac{X_{ \pm}}{r D \pi}, \quad \text { with } \quad X_{ \pm}=\frac{\zeta_{i_{1}} n_{1}+\zeta_{i_{2}} n_{2} \pm \sqrt{\left(\zeta_{i_{1}} n_{1}-\zeta_{i_{2}} n_{2}\right)^{2}+4 n_{1} n_{2}}}{2 n_{1} n_{2}}
$$

Note that $z_{+}-z_{-} \geq \frac{2}{\pi \sqrt{n_{1} n_{2}} r D}$. One can perform the same computations for a larger number of adjacent cells with "coinciding" roots: at the end one has to solve a polynomial equation in the variable $X$. The roots are always distinct and separated by a relative distance of order $\left(r D z_{0}\right)^{-1}$. Section 3.1 is devoted to the exact computation of the roots for an array of identical cells, which is a good example of such a situation.

In all the above computations we assumed $z_{0}=n \pi / l_{i}$ with positive $n$. However there are also $m$ roots located near zero. To find them we expand the sine and cosine functions in Eq. (33) and get to the first 
order in $z l$ a polynomial equation of degree $m$ in the variable $Z=r D l z^{2}$, where $l$ is the harmonic mean of the $l_{i}$. Hence we obtain $m$ roots of the form:

$$
z_{n}=\sqrt{\frac{Z_{n}}{r D l}}, \quad n=1, \ldots, m
$$

with $Z_{n}$ spanning the solutions of the polynomial equation. Note that we assumed $r D / l_{i} \gg 1$ hence one has $z l \ll 1$, which legitimates a posteriori the polynomial expansion. Furthermore, the first coefficients of the polynomial expansion are readily available from Eq. (33) and we get from them that:

$$
\sum_{n=1}^{m} Z_{n} \approx 2 m
$$

This formula is valid in the regime $r D / l \gg 1$ and its simplicity comes from the particular choice of $l$ we made (harmonic mean of the $l_{i}$ ). If one assumes that the roots $Z_{n}$ are approximately equispaced at small $n$, then one obtains immediately that the first roots $Z_{n}$, and hence $\lambda_{n}$, follow a $1 / m^{2}$ dependence on $m$.

From this analysis of the low permeability regime $\left(r D / l_{i} \gg 1\right.$ for all $\left.i\right)$ we can draw several conclusions, partly illustrated in Fig. 2.

- the $m$ first roots $(z l \ll 1)$ behave differently than the other ones. They typically spread over a distance $(r D l)^{-1 / 2}$.

The following points only apply to the other roots $(z l \gtrsim 1)$.

- all the roots increase from the limits $z_{0}=n \pi / l_{i}$ with the permeability of the inner barriers (a general mathematical proof of this statement is given in Sec. SM. III.3). The relative increase is of the first order in $\left(r D z_{0}\right)^{-1}$;

- very close roots associated to adjacent cells are coupled by the permeability of their barrier and separate from each other by a relative distance of order $\left(r D z_{0}\right)^{-1}$;

- very close roots associated to non-adjacent cells are not coupled to the first order in $(r D z)^{-1}$. The difficult case is when the two cells have the same length: then $n_{1}=n_{2}$ and the relative distance between the two roots is in the best case of order $\left(z_{0} r D\right)^{-2}$. In fact, it depends on the length of all other cells. For example, symmetric geometries typically lead to a relative distance between roots of order $\left(z_{0} r D\right)^{-\left|i_{2}-i_{1}\right|}$.

All the previous computations are somewhat schematic because we made a particular choice of geometry (same diffusion coefficients, same permeability and no relaxation at the outer boundaries) from the beginning. However, the above conclusions are globally still valid in the general case, with appropriate modifications. For example if one considers perfectly relaxing condition at the endpoints $\left(K_{ \pm}=\infty\right)$, then in the low-permeability limit the roots corresponding to the outer compartments are $z_{0}=(n+1 / 2) \pi / l_{i}$ $\left(i=1\right.$ or $m$ ), whereas the roots corresponding to the other compartments are $z_{0}=n \pi / l_{i}, 1<i<m$ (with an integer $n$ ). Thus one has to consider separately the case of the outer compartments depending on the conditions at the outer boundaries. We come back to the relaxing case in Sec. SM. II and Sec. SM. IV.5. Moreover, the case of heterogeneous diffusion coefficients is treated analytically in the simplest case of a bi-periodic structure in Sec. SM. V.

\subsection{Extensions}

The above analysis may be extended in many ways. First, one can consider more general boundary conditions. In particular, many experiments in heat conduction are done with one end of the system in contact with a heat source (acting as a constant heat flux or as a thermostat with a constant temperature). One should then replace our homogeneous outer boundary conditions $(7),(8)$ by inhomogeneous boundary conditions. The only difference is in the steady-state solution $(\lambda=0)$ which is easy to obtain, whereas the transient solution remains the same (see $[19,34])$. One is then often interested in the "critical time", i.e. the typical time required to reach the steady-state solution. More precisely, one definition of the critical time is the time at which the average temperature over the sample is equal to some fraction $\alpha<1$ of 
the average steady-state temperature over the sample. Other definitions and a thorough comparison of these definitions are detailed in $[46,47]$. This time is essentially given by the study of the first nonzero eigenvalue of the diffusion operator, for which we are able to obtain estimates with respect to the geometrical parameters of the medium (such as Eq. (38), which yields $\lambda \sim\left(r l m^{2}\right)^{-1}$, in the lowpermeability regime). The situation is different when the boundaries are subject to modulated heating, which is the case in geophysics and building design [13-17], and in photothermal measurements [11, 12]. One can still transform the problem into an homogeneous boundary problem but it requires adding a suitable source term to the diffusion equation [34]. In some cases the main mechanism of heat relaxation at the outer boundaries is not conduction-convection but radiation, with a non-linear $T^{4}$ heat flux [48]. Finally, when considering diffusion of ions in multilayer chemical system such as electrodes, one writes chemical equilibrium condition at the interfaces: the ratio of concentrations on both sides of the interface is equal to the partition coefficient $[7-9,49,50]$. This is another type of inner boundary condition, which leads to different $\mathcal{K}$ matrices, quite similar to the case of heterogeneous diffusion coefficients and no barriers.

Another possible generalization is the inclusion of bulk reaction rates inside the compartments. That is, to change Eq. (1) to a reaction-diffusion equation:

$$
\frac{\partial G}{\partial t}=D \nabla^{2} G+\mu G
$$

where $\mu$ may depend on space and $G$ [46]. If $\mu$ is constant, then one gets the solution of Eq. (40) by multiplying the solution of Eq. (1) by $\exp (\mu t)$. The case of piecewise constant $\mu\left(\mu=\mu_{i}\right.$ on $\left.\Omega_{i}\right)$ is slightly more complicated but may be easily incorporated into our computations. Such reaction-diffusion models may describe diffusion of molecules that can be trapped, killed, destroyed, or loose their activity [51$55]$ or, on the opposite, self-heating by temperature-induced oxidation [56] $(\mu>0)$. Other applications include ecology dynamics [57] and fabrication of multilayer foil materials $[58,59]$.

Last, one can consider other equations than the diffusion equation (1), for example:

- inhomogeneous Laplace (Poisson) equation: $\nabla(D \nabla \Psi)=F$,

- inhomogeneous Helmholtz $(s>0)$ or modified Helmholtz $(s<0)$ equations: $(s+\nabla D \nabla) \Psi=F$,

- inhomogeneous diffusion equation: $\frac{\partial \Psi}{\partial t}-\nabla(D \nabla \Psi)=F, \Psi(x, t=0)=U(x)$,

- inhomogeneous wave equation: $\frac{\partial^{2} \Psi}{\partial t^{2}}-\nabla(D \nabla \Psi)=F, \Psi(x, t=0)=U(x), \frac{\partial \Psi}{\partial t}(x, t=0)=V(x)$,

where $F, U, V$ are given functions, and with the boundary conditions (5), (6), (7), and (8). Thanks to the knowledge of the eigenmodes basis of the diffusion operator $\nabla(D \nabla)$, the above equations may be solved by decomposing $u$ and $F$ over this basis $[1,2]$.

The computational method that we presented is therefore relevant to many models and applications. In the Supplementary Material we discuss two particular examples: diffusion MRI (Sec. SM. I) and first exit time distribution (Sec. SM. II).

\section{Example: simple periodic geometry}

In this section, we illustrate the application of our general method to the case of a (finite) periodic structure which is relevant for various applications. Throughout this section, we assume that all $l_{i}, D_{i}$, $\kappa_{i, i+1}$ are the same (denoted $l, D, \kappa$ in the following). We apply the results of Sec. 2 and obtain the eigenmodes and eigenvalues $u_{n}, \lambda_{n}$. Similar computations for more complicated structures are presented in Sec. SM. V (bi-periodic geometry) and Sec. SM. VI (two-scale geometry).

\subsection{Eigenmodes}

We assume reflecting boundary conditions at the endpoints $\left(K_{ \pm}=0\right)$ and introduce the dimensionless parameters

$$
\alpha=\sqrt{\lambda / D} l \quad \text { and } \quad \tilde{r}=1 / \tilde{\kappa}=r D / l
$$


Then the transition matrix of the elementary block is simply

$$
\mathcal{M}=\mathcal{K} \mathcal{R}=\left[\begin{array}{cc}
\cos \alpha-\tilde{r} \alpha \sin \alpha \sin \alpha+\tilde{r} \alpha \cos \alpha \\
-\sin \alpha & \cos \alpha
\end{array}\right]
$$

and Eq. (19) on the spectrum becomes

$$
\mathcal{K}^{-1} \mathcal{M}^{m}\left[\begin{array}{l}
\alpha \\
0
\end{array}\right]=\epsilon\left[\begin{array}{l}
\alpha \\
0
\end{array}\right]
$$

Since the geometry is symmetric, we already know that $\epsilon= \pm 1$. Furthermore we use the results of Sec. 2.4 to compute $\mathcal{M}^{m}$ : first we apply Eq. (30) to define $\psi$ :

$$
\cos \psi=\cos \alpha-\frac{\tilde{r}}{2} \alpha \sin \alpha
$$

then from Eq. (29), we get

$$
\mathcal{M}^{m}=\left[\begin{array}{cc}
(\cos \alpha-\tilde{r} \alpha \sin \alpha) \frac{\sin m \psi}{\sin \psi}-\frac{\sin (m-1) \psi}{\sin \psi} & (\sin \alpha+\tilde{r} \alpha \cos \alpha) \frac{\sin m \psi}{\sin \psi} \\
-\sin \alpha \frac{\sin m \psi}{\sin \psi} & \cos \alpha \frac{\sin m \psi}{\sin \psi}-\frac{\sin (m-1) \psi}{\sin \psi}
\end{array}\right] .
$$

Equation (43) can be further simplified by using the fact that $\mathcal{K}\left[\begin{array}{l}1 \\ 0\end{array}\right]=\left[\begin{array}{l}1 \\ 0\end{array}\right]$. We thus have the simple condition

$$
\mathcal{M}^{m}\left[\begin{array}{l}
1 \\
0
\end{array}\right]=\epsilon\left[\begin{array}{l}
1 \\
0
\end{array}\right],
$$

which gives the equation on $\alpha$ (and thus on eigenvalues $\lambda$ )

$$
\sin \alpha \frac{\sin m \psi}{\sin \psi}=0 \text {. }
$$

This corresponds to two cases:

$-\sin \alpha=0$, that is $\alpha=j \pi$, with $j=0,1,2, \ldots$ We denote these solutions by $\alpha_{j, 0}$ if $j$ is even and $\alpha_{j, m}$ if $j$ is odd. The vector $\left[\begin{array}{l}1 \\ 0\end{array}\right]$ is an eigenvector of the matrix $\mathcal{M}$ with the eigenvalue $(-1)^{j}$, thus $\epsilon=(-1)^{j m}$.

$-\frac{\sin m \psi}{\sin \psi}=0$, which gives $m \psi=p \pi$, where $p \in\{1, \ldots, m-1\}$, and can be restated according to Eq. (44) as:

$$
\cos \alpha-\frac{\tilde{r}}{2} \alpha \sin \alpha=\cos p \pi / m, \quad p \in\{1, \ldots, m-1\} .
$$

For each value of $p$ this yields an infinite array of solutions that we will denote as $\alpha_{j, p}$, where the $j$ index means $j \pi \leq \alpha_{j, p}<(j+1) \pi(j=0,1, \ldots)$. We have $\mathcal{M}^{m}=(-1)^{p} \mathcal{I}_{2}$, therefore $\epsilon=(-1)^{p}$.

Figure 3 illustrates the solutions $\alpha_{j, p}$ in the case $m=4$ and $\tilde{r}=0.4$. One can see that the solutions are grouped in branches of $m$ values. Each branch begins at a multiple of $\pi$ and ends below the next one. The branches of even $j$ begin with $\psi=0(p=0)$ and increase with increasing $p$, whereas the odd $j$ branches begin with $\psi=\pi(p=m)$ and increase with decreasing $p$. Note that we discard the branches with negative $j$ because $\alpha \geq 0$ according to Eq. (41).

Note that $\alpha$ (or $j$ ) dictates the intra-compartment variation of the mode, whereas $\psi$ (or $p$ ) is related to its inter-compartment variation (as we explained in Sec. 2.4). In fact, the index $j$ is equal to the number of extrema of the mode in the first compartment (not counting the one at $x=0$ ). If one is interested in the inter-compartment variation only, for example by looking at the value of the mode at the beginning of each compartment, then $p$ represents the number of extrema of this variation over the whole interval. Moreover, the Courant nodal theorem (proved for our particular model in Sec. SM. III.4) states that each eigenmode changes sign $p+j m$ times. Figure 4 shows the first modes of an array of $m=4$ identical cells with impermeable outer barriers. The first two branches are represented. We have additionally plotted dots at the beginning of each compartment to make the inter-compartment variation more visible. 

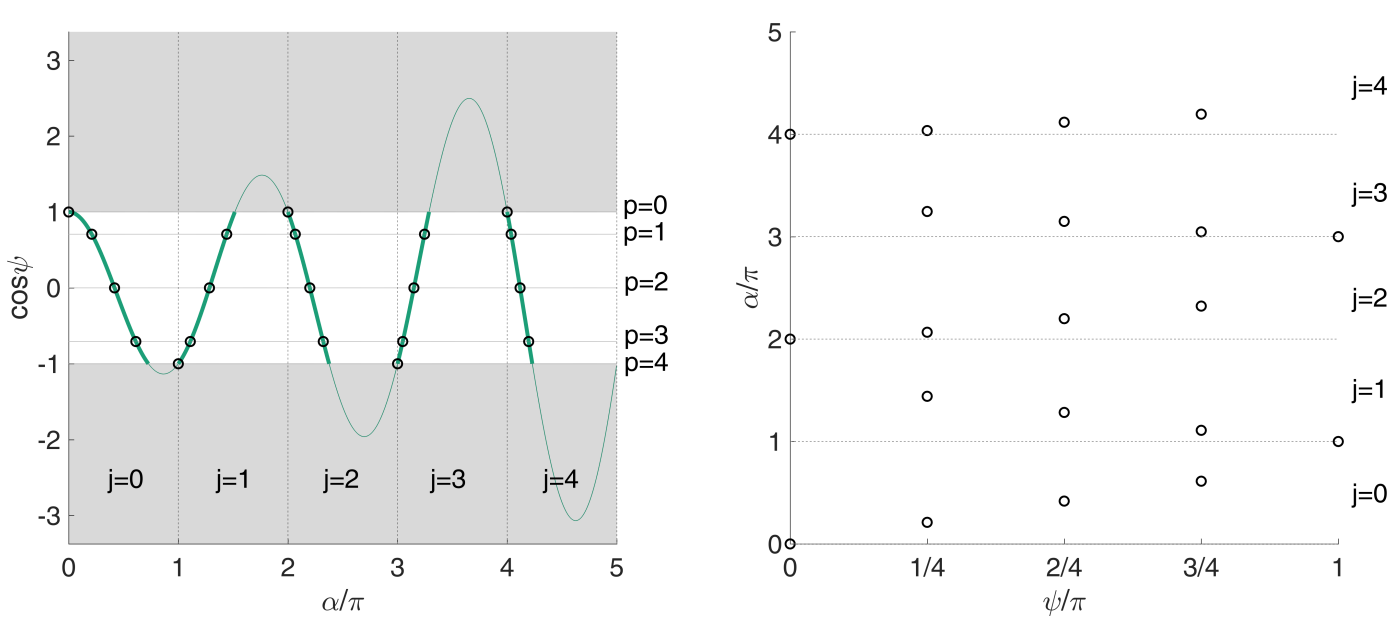

Fig. 3 (left) Plot of $\cos \psi=\cos \alpha-\frac{\tilde{r}}{2} \alpha \sin \alpha$ with $\tilde{r}=0.4$. Horizontal dotted lines indicate $\cos \psi=\cos p \pi / m, p=$ $0, \ldots, m$, with $m=4$ and the circles represent the solutions $\alpha_{j, p}$. (right) An equivalent representation is the plot of $\alpha_{j, p}$ versus $\psi_{p}=p \pi / m$. One can see branches beginning at $j \pi$ and ending below $(j+1) \pi$. As $\alpha$ increases, the graph of $\cos \psi$ crosses the $[-1 ; 1]$ interval with a steeper slope, which results in solutions closer to $j \pi$ as $j$ increases.
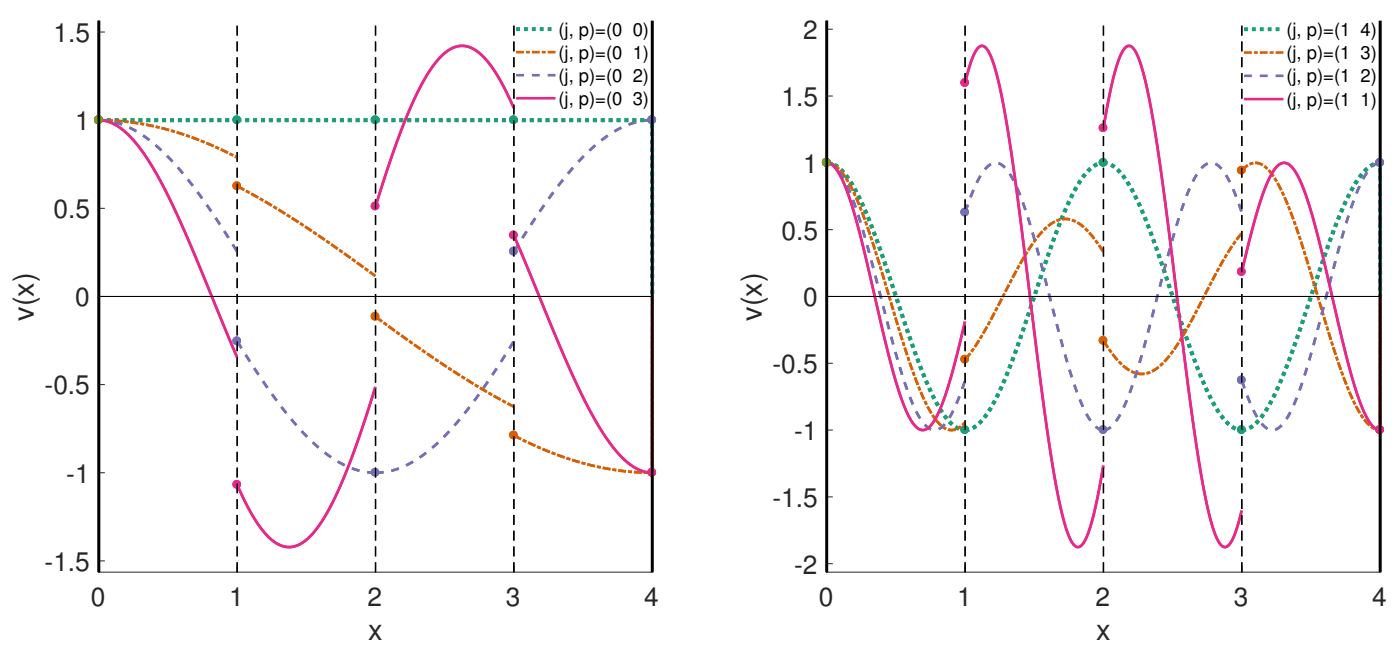

Fig. 4 Plot of the diffusion operator eigenmodes for the array of $m=4$ identical cells of length 1 with impermeable outer boundaries and $\tilde{r}=0.4$. (left) $j=0, p=0, \ldots, m-1$; (right) $j=1, p=m, \ldots, 1$. Note the discontinuities at the barriers which increase when $\alpha_{j, p}$ increases.

One can compare the results of this section with Bloch waves in solid state physics. Indeed the branches of solutions $\alpha_{j, p}$ are similar to energy bands, where $j$ and $p$ are analogous to the band index $n$ and the wavenumber $k$, respectively. This is no surprise because we are dealing with a (finite) periodic geometry. Although the periodicity is not expressed through an energy potential but boundary conditions, the mathematical framework is the same. This explains the striking similarity between Fig. 3 and energy band diagrams (where only the $k \geq 0$ half would be represented). 


\subsection{Computation of the norm}

Because the geometry is symmetric and the relaxation coefficients $K_{ \pm}$are equal to zero, one can transform the formula (24) of the normalization constant into

$$
\beta_{j, p}^{-2}=\frac{l}{2}\left|\left[\begin{array}{ll}
0 & 1
\end{array}\right] \frac{\mathrm{d} \mathcal{T}}{\mathrm{d} \alpha}\left[\begin{array}{l}
1 \\
0
\end{array}\right]\right|_{\alpha=\alpha_{j, p}}=\frac{l}{2}\left|\left[\begin{array}{ll}
0 & 1
\end{array}\right] \frac{\mathrm{d} \mathcal{M}^{m}}{\mathrm{~d} \alpha}\left[\begin{array}{l}
1 \\
0
\end{array}\right]\right|_{\alpha=\alpha_{j, p}}=\frac{l}{2}\left|\frac{\mathrm{d}}{\mathrm{d} \alpha}\left(\sin \alpha \frac{\sin (m \psi)}{\sin \psi}\right)\right|_{\alpha=\alpha_{j, p}} .
$$

Now we use Eq. (47), which leads us to distinguish the two cases as above:

$-\sin \alpha=0$ : it corresponds to $\alpha=j \pi$, with a positive integer $j$ (recall that we discard $\alpha=0$ ). Then $\cos \psi=(-1)^{j}$ and $\frac{\sin m \psi}{\sin \psi}=m(-1)^{j(m-1)}$. We conclude that the norm of the mode is:

$$
\beta_{j, p}^{2}=\frac{2}{m l}
$$

$-\frac{\sin m \psi}{\sin \psi}=0$ : it corresponds to $\alpha_{j, p}(\psi=p \pi / m), p=1, \ldots, m-1$ and $j=0,1, \ldots$ In this case, the derivative in Eq. (49) is easily computed by the chain rule:

$$
\begin{aligned}
& \frac{\mathrm{d}}{\mathrm{d} \alpha}\left(\frac{\sin m \psi}{\sin \psi}\right)=\frac{\mathrm{d} \cos \psi}{\mathrm{d} \alpha} \frac{\mathrm{d} \psi}{\mathrm{d} \cos \psi} \frac{\mathrm{d}}{\mathrm{d} \psi}\left(\frac{\sin m \psi}{\sin \psi}\right) \\
& =-\left(\sin \alpha\left(1+\frac{\tilde{r}}{2}\right)+\frac{\tilde{r}}{2} \alpha \cos \alpha\right)\left(\frac{-1}{\sin \psi}\right) \frac{m \cos m \psi \sin \psi-\sin m \psi \cos \psi}{\sin ^{2} \psi}
\end{aligned}
$$

which by evaluation at $\alpha_{j, p}$ yields:

$$
\beta_{j, p}^{2}=\frac{2}{m l} \frac{\sin ^{2} p \pi / m}{\sin \alpha_{j, p}\left(\sin \alpha_{j, p}\left(1+\frac{\tilde{r}}{2}\right)+\frac{\tilde{r}}{2} \alpha_{j, p} \cos \alpha_{j, p}\right)} .
$$

\section{Implementation and Applications}

\subsection{Numerical Implementation}

From a numerical point of view, the computational steps are the following: (i) to compute the transition matrix $\mathcal{M}_{i, i+1}$ in Eq. (17) for each compartment; (ii) to apply Eq. (20) to get the complete transition matrix; (iii) to solve Eq. (22) to get the spectrum of the diffusion operator; each solution of Eq. (22) determines one eigenvalue whereas Eqs. (16) and (18) yield the coefficients $a_{i}^{l}, b_{i}^{l}, k=1, \ldots, m$ for each (non-normalized) mode; (iv) to compute the normalization constant; combined with Eq. (11) it allows one to compute the eigenmode at any point of the interval.

Steps (i) and (ii) are easy and fast since we are dealing with $2 \times 2$ matrices. Step (iv) can be done either with Eq. (24), which involves a numerical derivative, or by a direct computation, using:

$$
\int_{0}^{l}(a \cos (k x)+b \sin (k x))^{2} \mathrm{~d} x=\frac{\left(a^{2}+b^{2}\right) l}{2}+\frac{\left(a^{2}-b^{2}\right)}{4 k} \sin (2 k l)+\frac{a b}{4 k}(1-\cos (2 k l)) .
$$

The most complicated and time-consuming step is (iii). As we explained in Sec. 2.5, two or more solutions of Eq. (22) may be very close to each other in the case of low-permeability barriers (typically $\kappa \ll D / l)$. The estimates we derived allow us to localize the roots that speeds up the computation. This is the crucial point and one of the major practical achievements of the paper. This numerical improvement allows us to detect very close zeros (as those shown in Fig. 2) and to compute the eigenmodes of the diffusion operator in heterogeneous structures with hundreds of barriers. Moreover, Fig. 2 illustrates an interesting property of $F_{m}\left(z ; l_{1}, \ldots, l_{m}\right)$ as a function of $z$ : two local extrema are apparently always separated by a zero. Although we have no mathematical proof for this observation, it is very helpful because it allows us to detect pairs of close zeros by the change of sign of the derivative of the function, which may take place on a much larger scale than the change of sign of the function itself. One can 
also take advantage of the Courant nodal theorem (which is proven for our particular model in Sec. SM. III.4): the $n$-th eigenmode has $n$ nodal domains (connected components on which the eigenmode has a constant sign), or equivalently, the $n$-th eigenmode changes sign $n-1$ times (possibly at the barriers). This can be used as an efficient test to check a posteriori that no eigenvalue is missed.

In practice, the standard floating-point precision limits the relative accuracy of a numerical computation to about $10^{-15}$. Let us assume that we are dealing with a geometry such that two eigenvalues $\lambda_{1}$ and $\lambda_{2}$ are much closer than this limit; for example they coincide up to $10^{-20}$. With the above tricks we are still able to detect those roots and even to compute accurately their position and spacing. However, the subsequent computations performed on $\lambda_{1}$ and $\lambda_{2}$ (for example, the computation of the eigenmodes or their norm) treat $\lambda_{1}$ and $\lambda_{2}$ as equal numbers. Even worse: the closeness of $\lambda_{1}$ and $\lambda_{2}$ is related to the very fast local variations of $F(\lambda)$ with $\lambda$, and as a consequence of the coefficients $\left(a_{i}^{l}, b_{i}^{l}\right)$ and of the norm of the eigenmode. Therefore it is very difficult to compute accurately these quantities for two eigenmodes corresponding to very close eigenvalues. The estimates derived in Sec. 2.5 can be used to detect a priori such situations in which the spectral decomposition can numerically fail.

If one is interested in the diffusion propagator (23) or related quantities, the infinite collection of eigenmodes has to be truncated. This is done by sorting the eigenvalues $\lambda_{n}$ in ascending order and then cutting off the ones such that $\lambda_{n} t \gg 1$, where $t$ is the smallest diffusion time for which the computation is needed. The precise choice of the truncation threshold is a compromise between precision and speed of computation. Practically, one can check the validity of the truncation by re-doing the computation with a higher threshold and then comparing the two results.

We have implemented the proposed method for an arbitrary configuration of barriers and diffusion coefficients as a Matlab code. The numerical results presented in the Supplementary Materials were obtained on a basic laptop computer by using this code. The code can be sent upon request.

\subsection{Application to diffusion MRI}

Diffusion of spin-bearing particles (such as nuclei of hydrogen atoms in water molecules) may be surveyed by diffusion magnetic resonance imaging (dMRI), which is a powerful imaging technique with many biomedical applications [60-63]. From the knowledge of the diffusion propagator one can access the dMRI signal under the so-called Narrow-Pulse Approximation (NPA), thus motivating numerous theoretical and experimental works on diffusion in complex geometries. As explained previously, restricted diffusion in simple domains such as slab, cylinder, sphere, can be treated analytically [64-67]. In contrast, most works devoted to multi-layered systems with semi-permeable barriers are numerical. Tanner took advantage of the simple expression of the Laplace eigenmodes in a slab geometry to study a finite periodic repetition of semi-permeable barriers [68]. The same method was applied later by Kuchel and Durrant to unevenly spaced membranes [69]. These approaches were generalized by Grebenkov with a matrix formalism allowing efficient computation of the signal in general multi-layered planar, cylindrical or spherical structures, without the NPA restriction [38]. Powles and co-workers proposed in [70] an opposite approach based on the (one-dimensional) analytical solution of $G$ for one semi-permeable barrier extended to several barriers by multiple reflections. Other numerical techniques such as a finite differences method were reported [71]. The first analytical expression of the dMRI signal in a one-dimensional geometry with periodic permeable barriers was provided by Sukstanskii et al. [72]. Relying on the periodicity of the system they computed directly the signal in Laplace domain without having to derive the diffusion propagator. Unevenly spaced membranes were treated in [73, 74] from the analytical solution for one membrane and under the assumption that the diffusing time is sufficiently short so that the layers are independent. Note that in contrast to almost all previously cited works the analysis performed in [74] does not confine to infinitely narrow pulses. Finally, Novikov et al. studied the effect of randomly placed semi-permeable barriers on the diffusive motion $[75,76]$. Using a renormalization group technique, they obtained structural universality classes characterized by the disorder introduced by the barriers, which in turn govern the long-time asymptotic behavior of the mean square displacement.

In the general case, the signal is obtained by solving the Bloch-Torrey equation for the local magnetization $m(x, t)$ :

$$
\frac{\partial m}{\partial t}=D \nabla^{2} m+i \gamma g x f(t) m,
$$


where $D$ is the diffusion coefficient, $\gamma$ the gyromagnetic ratio of the nuclei, $g$ the magnetic field gradient and $f(t)$ a customizable temporal profile [60-62]. In our one-dimensional geometry, the signal is then given by

$$
S(t)=\frac{1}{L} \int_{0}^{L} m(x, t) \mathrm{d} x .
$$

The method developed in Sec. 2 for computing the diffusion operator eigenmodes allows us to calculate the signal analytically for infinitely narrow gradient pulses, or numerically for arbitrary pulse sequences (such as the one in Fig. 5). In particular, this method generalizes earlier approaches [68, 71-73] and opens unprecedented opportunities for studying more sophisticated configurations of barriers such as microstructures inside larger scale structures.

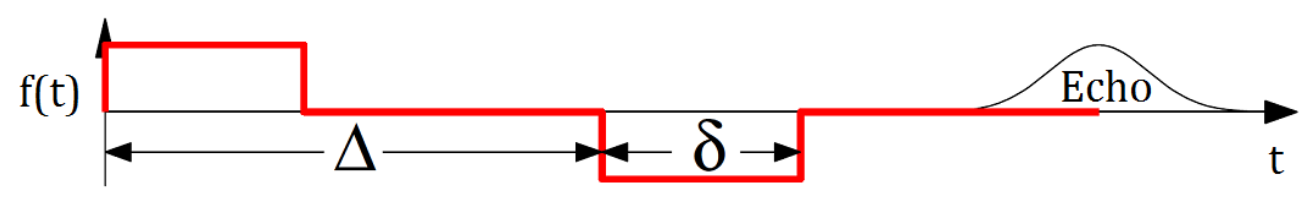

Fig. 5 Temporal profile $f(t)$ for Pulsed-Gradient-Spin-Echo (PGSE) experiments. The Narrow-Pulse Approximation (NPA) is the limit case $\delta \rightarrow 0$ while $\gamma g \delta$ remains constant.

The computations are detailed in Sec. SM. I. We explain how one can obtain the dMRI signal from the Fourier transform of the eigenmodes $u_{n}$ in the so-called narrow pulse regime, then we derive the expression of the signal for the periodic geometry presented in Sec. 3. We discuss the effect of the permeability of the barriers on the dMRI signal in the regimes of short and long diffusion time. In particular, we obtain a scaling law of the form $\tilde{\kappa} t /(\tilde{\kappa}+1)$ involving $t$ and $\tilde{\kappa}=\kappa l / D$, which is valid in the long time regime $\left(t \gg l^{2} / D\right)$. Computations for more sophisticated geometries are presented in Sec. SM. IV (relaxation at the outer boundaries), SM. V (bi-periodic geometry), and SM. VI (two-scale geometry).

\subsection{First exit time distribution}

Another application of the diffusion operator eigenmodes is the computation of the first exit time distribution. First exit times are a particular case of first passage phenomena, which find many applications in physics, chemistry, biology, or economy. In particular, one-dimensional models are relevant to a wide variety of phenomena in which an event is triggered when a fluctuating variable reaches a given threshold (examples include avalanches, neuron firing, or sell/buy orders) as well as diffusion controlled reactions such as fluorescence quenching or predation [77, 78]. In general planar domains, exit times were thoroughly investigated in the so-called "narrow-escape limit" [79] and few results are available for arbitrary escape areas $[80,81]$.

For this purpose, let us consider perfectly relaxing conditions at the outer boundaries of the interval $[0, L]: K_{ \pm}=\infty$. Then the quantity

$$
\int_{0}^{L} G\left(x \rightarrow x^{\prime}, \tau\right) \mathrm{d} x^{\prime}
$$

represents the probability of not reaching the outer boundaries for a particle starting at $x$, up to the time $\tau$. In other words, if one denotes by $T_{x}$ the random variable equal to the first exit time of a particle starting at $x$, then the tail distribution and the probability density of $T_{x}$ are respectively given by:

$$
\begin{aligned}
& \mathbb{P}\left(T_{x}>\tau\right)=\int_{0}^{L} G\left(x \rightarrow x^{\prime}, \tau\right) \mathrm{d} x^{\prime}=\sum_{n=1}^{\infty} e^{-\lambda_{n} \tau} u_{n}(x)\left(\int_{0}^{L} u_{n}\left(x^{\prime}\right) \mathrm{d} x^{\prime}\right) \\
& \rho_{T_{x}}(\tau)=\frac{\mathbb{P}\left(\tau<T_{x}<\tau+\mathrm{d} \tau\right)}{\mathrm{d} \tau}=\sum_{n=1}^{\infty} \lambda_{n} e^{-\lambda_{n} \tau} u_{n}(x)\left(\int_{0}^{L} u_{n}\left(x^{\prime}\right) \mathrm{d} x^{\prime}\right) .
\end{aligned}
$$


The computations are detailed in Sec. SM. II. We rely on the computation of the eigenmodes for a periodic geometry with perfectly relaxing outer boundaries performed in Sec. SM. IV and obtain the first exit time distribution for this structure. We study the limit of a large number of barriers (where the size $L$ of the large interval remains constant). Similarly to the computation of the dMRI signal, we obtain a scaling law of the form $\tilde{\kappa} t /(\tilde{\kappa}+1)$. Then we turn to irregular geometries where $l_{i}$ and $\kappa_{i, i+1}$ are randomly distributed and we observe the same scaling law, with a new definition for $\tilde{\kappa}$ which depends on permeabilities and positions of the barriers. Numerical computations show a very good agreement even for a moderate number of barriers $(m \approx 10)$. Moreover, we analyze the regime of very low permeability, where the diffusive motion can be replaced by a discrete hopping model, and exhibit a perfect agreement with previously obtained results.

\section{Conclusion}

We presented an efficient method to compute the eigenmodes of the diffusion operator on a one-dimensional interval segmented by semi-permeable barriers, which in turn give access to the diffusion propagator. One can then compute several diffusion-related quantities such as the dMRI signal for any pulse sequence or the first exit time distribution.

Although the general matrix formalism is applicable to other multi-layered structures such as concentric cylindrical or spherical shells [38], the main analytical simplifications follow from the translation invariance of the Laplacian eigenmodes which is specific to one-dimensional models. In particular we derived some estimates that help us to accurately compute the eigenvalues, even when they are extremely close to each other. This is the crucial numerical step that allowed us to deal with heterogeneous structures with hundreds of semi-permeable barriers. This efficient method opens unprecedented opportunities to investigate the impact of microstructure onto diffusive motion.

Acknowledgements We acknowledge the support under Grant No. ANR-13-JSV5-0006-01 of the French National Research Agency. 


\section{Supplementary Material for the article "Diffusion across semi-permeable barriers: spectral properties, efficient computation, and applications"}

\section{SM. I Computation of the dMRI Signal}

\section{SM. I.1 General case}

For a general geometry and an arbitrary pulse sequence one may solve numerically the Bloch-Torrey equation (53) by decomposing $m(x, t)$ over the diffusion operator eigenmodes basis $\left(u_{n}\right)_{n \in \mathbb{N}}$ :

$$
m(x, t)=\sum_{n=1}^{\infty} m_{n}(t) u_{n}(x), \quad \text { with } \quad m_{n}(t)=\int_{0}^{L} u_{n}^{*}(x) m(x, t) \mathrm{d} x
$$

where the asterisk denotes complex conjugation $[62,82]$. Truncating the decomposition (S1) to a finite number of terms $n_{\max }$, one can represent the solution of the Bloch-Torrey equation as a vector:

$$
\mathbf{m}(t)=\left(\begin{array}{c}
m_{1}(t) \\
m_{2}(t) \\
\vdots \\
m_{n_{\max }}(t)
\end{array}\right)
$$

The Bloch-Torrey equation can then be rewritten as

$$
\frac{\partial \mathbf{m}}{\partial t}=-\Lambda \mathbf{m}+i \gamma g f(t) B \mathbf{m}
$$

with the following matrices:

$$
\begin{aligned}
& \Lambda_{n, n^{\prime}}=\int_{0}^{L} u_{n}^{*}(x)\left(-D \nabla^{2} u_{n^{\prime}}(x)\right) \mathrm{d} x=\lambda_{n} \delta_{n, n^{\prime}} \\
& B_{n, n^{\prime}}=\int_{0}^{L} u_{n}^{*}(x) x u_{n^{\prime}}(x) \mathrm{d} x
\end{aligned}
$$

where $\delta_{n, n^{\prime}}$ is the Kronecker symbol: $\delta_{n, n^{\prime}}=1$ if $n=n^{\prime}$ and $\delta_{n, n^{\prime}}=0$ otherwise. Moreover, one can write

$$
S(t)=\frac{1}{L} \int_{0}^{L} m(x, t) \mathrm{d} x=(\mathbf{a} \cdot \mathbf{m}(t))=\sum_{n=0}^{n_{\max }} a_{n} m_{n}(t), \quad \text { with } \quad a_{n}=\frac{1}{L} \int_{0}^{L} u_{n}(x) \mathrm{d} x
$$

When the gradient profile $f(t)$ is made of two rectangular pulses of duration $\delta$, separated by time $\Delta$ (Fig. $5)$, the corresponding PGSE signal is obtained by computing the following scalar product:

$$
S=\mathbf{a} \cdot\left[e^{-(\Delta-\delta) \Lambda} e^{-\delta(\Lambda+i \gamma g B)} e^{-(\Delta-\delta) \Lambda} e^{-\delta(\Lambda-i \gamma g B)}\right] \mathbf{m}(t=0)
$$

with matrix exponentials in square brackets. More generally, approximating the profile $f(t)$ by a piecewise constant function, one can accurately compute the dMRI signal for an arbitrary profile $f(t)[38,62,82]$. The initial condition for the magnetization is often uniform, $m(t=0)=1 / L$, in which case $\mathbf{m}(t=0)=\mathbf{a}$. 
SM. I.2 NPA approximation for an array of identical cells and reflecting conditions at the outer boundaries

The Narrow-Pulse Approximation (NPA) is the limit $\delta \rightarrow 0$ while $\gamma g \delta$ remains constant. In this regime the signal is directly linked to the diffusion propagator $G$ by

$$
S(\Delta)=\int_{0}^{L} \int_{0}^{L} \rho\left(x_{0}\right) G\left(x_{0} \rightarrow x, \Delta\right) \cos \left(\gamma g \delta\left(x-x_{0}\right)\right) \mathrm{d} x \mathrm{~d} x_{0},
$$

where $\rho\left(x_{0}\right)$ is the initial spin density [60-62]. The spectral decomposition (23) yields

$$
S(\Delta)=\sum_{n=1}^{\infty} e^{-\lambda_{n} \Delta} \int_{0}^{L} \int_{0}^{L} \rho\left(x_{0}\right) u_{n}\left(x_{0}\right) u_{n}(x) \cos \left(\gamma g \delta\left(x-x_{0}\right)\right) \mathrm{d} x \mathrm{~d} x_{0},
$$

If the initial density is uniform $\rho\left(x_{0}\right)=1 / L$, the symmetry between $x$ and $x_{0}$ leads to the following simplification:

$$
S(\Delta)=\frac{1}{L} \sum_{n=1}^{\infty} e^{-\lambda_{n} \Delta}\left|\int_{0}^{L} u_{n}(x) e^{i \gamma g \delta x} \mathrm{~d} x\right|^{2} .
$$

This formula is the basis of the NPA and was initially introduced in [64] to study the signal coming from a single isolated interval. Later the effect of semi-permeable barriers was numerically studied in [68] for the most simple one-dimensional geometry where all $l_{i}, D_{i}, \kappa_{i, i+1}$ are the same (denoted $l, D, \kappa$ in the following).

In this section we apply the results of Sec. 3 and extend the results of Ref. [68]. In addition to Sec. 3 we compute the Fourier transform of the modes which gives us the signal $S$. In Sec. SM. IV we extend this computation to relaxing conditions at the outer boundaries. A more complicated geometry consisting of a microstructure inside a larger scale structure is treated in Sec. SM. VI.

We temporarily use the subscript $k$ instead of $i$ for the compartments in order to avoid any confusion with the imaginary unit $i=\sqrt{-1}$. As previously we use the position of the barrier to the left as the origin in the formula (11) of the eigenmodes. This means that we have to compute integrals of the form:

$$
\begin{aligned}
& \int_{0}^{l_{k}} e^{i \gamma g \delta x} \cos \left(x \sqrt{\lambda / D_{k}}\right) \mathrm{d} x=\frac{l_{k}}{2}\left(\frac{e^{i\left(\gamma g \delta+\sqrt{\lambda / D_{k}}\right) l_{k}}-1}{i l_{k}\left(\gamma g \delta+\sqrt{\lambda / D_{k}}\right)}+\frac{e^{i\left(\gamma g \delta-\sqrt{\lambda / D_{k}}\right) l_{k}}-1}{i l_{k}\left(\gamma g \delta-\sqrt{\lambda / D_{k}}\right)}\right) \\
& \int_{0}^{l_{k}} e^{i \gamma g \delta x} \sin \left(x \sqrt{\lambda / D_{k}}\right) \mathrm{d} x=\frac{l_{k}}{2 i}\left(\frac{e^{i\left(\gamma g \delta+\sqrt{\lambda / D_{k}}\right) l_{k}}-1}{i l_{k}\left(\gamma g \delta+\sqrt{\lambda / D_{k}}\right)}-\frac{e^{i\left(\gamma g \delta-\sqrt{\lambda / D_{k}}\right) l_{k}}-1}{i l_{k}\left(\gamma g \delta-\sqrt{\lambda / D_{k}}\right)}\right) .
\end{aligned}
$$

We denote by $\mathcal{L}_{k}$ the row vector whose components are the above integrals. The Fourier transform of the eigenmode $v$ is then simply

$$
\int_{0}^{L} v(x) e^{i \gamma g \delta x} \mathrm{~d} x=\sum_{k=1}^{m} e^{i \gamma g \delta x_{k-1, k}} \mathcal{L}_{k}\left[\begin{array}{c}
a_{k}^{l} \\
b_{k}^{l}
\end{array}\right] .
$$

Now we apply this general formula to our finite periodic geometry. The sum can be simplified because all $\mathcal{L}_{k}$ are the same:

$$
\mathcal{L}=\frac{l}{2}\left[-i\left(\frac{e^{i(q-\alpha)}-1}{q-\alpha}+\frac{e^{i(q+\alpha)}-1}{q+\alpha}\right)\left(\frac{e^{i(q-\alpha)}-1}{q-\alpha}-\frac{e^{i(q+\alpha)}-1}{q+\alpha}\right)\right],
$$

where $q=\gamma g \delta l$. Moreover $x_{k-1, k}=(k-1) l$ so we can rewrite the sum (S5):

$$
\begin{aligned}
\int_{0}^{L} v(x) e^{i \gamma g \delta x} \mathrm{~d} x & =\sum_{k=1}^{m} e^{i(k-1) q} \mathcal{L}\left[\begin{array}{l}
a_{k}^{l} \\
b_{k}^{l}
\end{array}\right]=\mathcal{L} \sum_{k=0}^{m-1} e^{i k q} \mathcal{M}^{k}\left[\begin{array}{l}
1 \\
0
\end{array}\right] \\
& =\mathcal{L}\left(\mathcal{I}_{2}-e^{i q} \mathcal{M}\right)^{-1}\left(\mathcal{I}_{2}-e^{i m q} \mathcal{M}^{m}\right)\left[\begin{array}{l}
1 \\
0
\end{array}\right]=\left(1-(-1)^{p} e^{i m q}\right) \mathcal{L}\left(\mathcal{I}_{2}-e^{i q} \mathcal{M}\right)^{-1}\left[\begin{array}{l}
1 \\
0
\end{array}\right]
\end{aligned}
$$


where we have used Eq. (46) with $\epsilon=(-1)^{p}$. We can simplify the matrix product further with the remark that the comatrix operation is linear for $2 \times 2$ matrices, and that $\operatorname{det} \mathcal{M}=1$, so that

$$
\mathcal{L}\left(\mathcal{I}_{2}-e^{i q} \mathcal{M}\right)^{-1}\left[\begin{array}{l}
1 \\
0
\end{array}\right]=\frac{\mathcal{L}\left(\mathcal{I}_{2}-e^{i q} \mathcal{M}^{-1}\right)}{\operatorname{det}\left(\mathcal{I}_{2}-e^{i q} \mathcal{M}\right)}\left[\begin{array}{l}
1 \\
0
\end{array}\right]=\frac{\mathcal{L}\left(\mathcal{I}_{2}-e^{i q} \mathcal{R}^{-1}\right)}{\operatorname{det}\left(\mathcal{I}_{2}-e^{i q} \mathcal{M}\right)}\left[\begin{array}{l}
1 \\
0
\end{array}\right] .
$$

From the knowledge of the trace and determinant of the matrix $\mathcal{M}$ we compute

$$
\operatorname{det}\left(\mathcal{I}_{2}-e^{i q} \mathcal{M}\right)=-2 e^{i q}(\cos \psi-\cos q)
$$

Furthermore,

$$
\mathcal{L}\left(\mathcal{I}_{2}-e^{i q} \mathcal{R}^{-1}\right)\left[\begin{array}{l}
1 \\
0
\end{array}\right]=-2 i e^{i q}(\cos \alpha-\cos q) \frac{q l}{q^{2}-\alpha^{2}}
$$

Putting all the pieces together yields

$$
\int_{0}^{L} v_{j, p}(x) e^{i \gamma g \delta x} \mathrm{~d} x=e^{i m q / 2} \frac{i q l\left(e^{-i m q / 2}-(-1)^{p} e^{i m q / 2}\right) \frac{\cos q-\cos \alpha_{j, p}}{\cos q-\cos p \pi / m}}{q^{2}-\alpha_{j, p}^{2}} .
$$

Note that the ratio is either real ( $p$ even) or imaginary ( $p$ odd) which is consistent with the symmetry or anti-symmetry of the mode (see Sec. 2.3).

SM. I.3 Complete expression of the signal

Let us summarize our results. In the array of $m$ identical cells one has $D_{i}=D$ and $l_{i}=l, i=1, \ldots, m$. We thus introduce the dimensionless time $t=D \Delta / l^{2}$, where $\Delta$ is the diffusion time (see Fig. 5 ), and $q=\gamma g \delta l$. The combination of the previous results yields the formula:

$$
\begin{aligned}
S= & \frac{2(1-\cos m q)}{(m q)^{2}}+\sum_{j=1}^{\infty} \frac{4 q^{2}\left(1-(-1)^{j m} \cos m q\right)}{m^{2}\left(q^{2}-(j \pi)^{2}\right)^{2}} e^{-(j \pi)^{2} t} \\
& +\sum_{j=0}^{\infty} \sum_{p=1}^{m-1} \frac{2 l q^{2}}{m} \frac{1-(-1)^{p} \cos m q}{(\cos q-\cos p \pi / m)^{2}}\left(\frac{\cos q-\cos \alpha_{j, p}}{q^{2}-\alpha_{j, p}^{2}}\right)^{2} \beta_{j, p}^{2} e^{-\alpha_{j, p}^{2} t}
\end{aligned}
$$

where $\beta_{j, p}^{2}$ is given by Eq. (51).

If $m=1$, there is no double sum on the second line of Eq. (S9), and one retrieves the well-known result by Tanner [64]:

$$
S_{1}(q, t)=\frac{2(1-\cos q)}{q^{2}}+\sum_{j=1}^{\infty} \frac{4 q^{2}\left(1-(-1)^{j} \cos q\right)}{\left(q^{2}-(j \pi)^{2}\right)^{2}} e^{-(j \pi)^{2} t} .
$$

The opposite limit $m \rightarrow \infty$ was the motivation of the subsequent article by Tanner [68] and was derived analytically in [72]. When $m \rightarrow \infty$, each term of the sum in Eq. (S9) vanishes except the ones for which $\cos p \pi / m$ is close to $\cos q$. Let us write

$$
q=2 k \pi+p_{0} \pi / m+\epsilon / m, \quad p_{0} \in\{0, \ldots, m-1\}, \quad 0 \leq \epsilon<\pi .
$$

Then we have:

$$
\frac{1-(-1)^{p} \cos (m q)}{m^{2}(\cos q-\cos p \pi / m)^{2}} \approx \frac{1-(-1)^{p_{0}-p} \cos \epsilon}{\pi^{2} \sin ^{2}(q)\left(p_{0}-p+\epsilon / \pi\right)^{2}} .
$$

To get the signal in the $m \rightarrow \infty$ limit, we thus have to compute the following sum:

$$
\sum_{p=-\infty}^{\infty} \frac{1}{\pi^{2}} \frac{1-(-1)^{p} \cos \epsilon}{(p+\epsilon / \pi)^{2}}=1 .
$$


The new equation on $\alpha$ is

$$
\cos \psi=\cos \alpha-\frac{\tilde{r}}{2} \alpha \sin \alpha=\cos q
$$

and the expression of the signal becomes

$$
S_{\infty}(q, t, \tilde{\kappa})=\frac{2 q^{2}}{\tilde{\kappa}} \sum_{n=1}^{\infty} \frac{\alpha_{n}{ }^{2} \sin \alpha_{n} e^{-\alpha_{n}{ }^{2} t}}{\left(\alpha_{n}{ }^{2}-q^{2}\right)^{2}\left((2 \tilde{\kappa}+1) \sin \alpha_{n}+\alpha_{n} \cos \alpha_{n}\right)} .
$$

This is exactly the formula derived in [72] by the computation of the Laplace transform of $\int G\left(x_{0} \rightarrow\right.$ $x) e^{i \gamma g \delta\left(x-x_{0}\right)} \mathrm{d} x_{0}$ on an infinite periodic geometry. Note that although the geometry is infinite and thus the spectrum of the diffusion operator is continuous, the signal is expressed in terms of a discrete set of eigenvalues because of Eq. (S11): the Fourier transform selects only the modes that globally oscillate at the wavenumber $q$ (recall that $\alpha$ only describes the intra-block oscillations, whereas the global behavior of the mode is dictated by $\psi$, according to Eq. (31)). This is consistent with the discreteness of the spectrum of the Airy operator $D \frac{\mathrm{d}^{2}}{\mathrm{~d} x^{2}}+i \gamma g x$ on any (bounded or unbounded) interval segmented by semi-permeable barriers $[74,83]$. As a consequence, one has to compute $\alpha_{n}, n=1,2, \ldots$ for each value of $q$, in contrast to the finite geometry where the spectrum depends only on the geometry and needs to be calculated only once. This is an important numerical advantage of the finite geometry over the infinite one because the computation of the spectrum is one of the most time-consuming step (as explained in Sec.2.5 and 4.1).

SM. I.4 Discussion: dependence of the signal on the permeability

In this section we study the diffusion operator eigenvalues and the signal in various regimes in order to show the dependence of the signal on the dimensionless permeability of the inner barriers, $\tilde{\kappa}$, which characterizes the microstructure. In biological tissues, one has typically: $D \sim 1 \mu \mathrm{m}^{2} / \mathrm{ms}, l=1-100 \mu \mathrm{m}$, $\kappa \sim 10^{-3}-1 \mu \mathrm{m} / \mathrm{ms}$, and the experimental range of diffusion time is about $\Delta \sim 10-10^{3} \mathrm{~ms}$. Thus we have the following ranges of variation for our dimensionless parameters: $\tilde{\kappa} \sim 10^{-3}-10^{2}$ and $t \sim 10^{-3}-10^{3}$.

In the limit $\tilde{\kappa} \rightarrow \infty$, one obviously recovers the signal associated to the whole interval of length $m l$ with no barriers, whereas in the opposite limit $\tilde{\kappa} \rightarrow 0$ one gets the signal (S10) associated to one interval of length $l$ (we detail the mathematical proof in Sec. SM. VII). In other words

$$
S(m, q, t, \tilde{\kappa}) \underset{\tilde{\kappa} \rightarrow \infty}{\longrightarrow} S_{1}\left(m q, t / m^{2}\right) \quad \text { and } \quad S(m, q, t, \tilde{\kappa}) \underset{\tilde{\kappa} \rightarrow 0}{\longrightarrow} S_{1}(q, t) .
$$

We are interested in the transition from one limit to the other, that is the dependence of the signal on the permeability. Expansions of $\alpha_{j, p}$ at low and high permeability are derived in Sec. SM. VIII. They show that the transition from $\tilde{\kappa}=0$ to $\tilde{\kappa}=\infty$ does not occur at one fixed value of $\tilde{\kappa}$ but depends on the branch of eigenvalues that we consider. Typically for the branch $j$ the transition occurs at $\tilde{\kappa} \sim j \pi / 2$ if $j>0$. As we have already seen, the $j=0$ branch is particular and exhibits a $\tilde{\kappa}^{1 / 2}$ dependence at low $\tilde{\kappa}$ (see Eqs. (S14) and (S87)). In order to refine our analysis we distinguish long-time and short-time regimes.

\section{SM. I.4.1 Long-time regime}

In the limit $t \rightarrow \infty$, all the modes with non-zero eigenvalues vanish and we are left with

$$
S=\frac{2(1-\cos m q)}{(m q)^{2}},
$$

which is a well-known formula [64]. Note that relaxation at the outer boundaries would lead to zero signal in the long-time limit because $\lambda=0$ would not be an eigenvalue of the diffusion operator anymore. As expected at long times the details of the geometry are averaged out and the signal depends only on the length of the whole interval, $L=m l$. The next terms are given by the first solutions of the $j=0$ branch. Let us study Eq. (48) at small $\alpha, \psi$. Expanding the sine and cosine functions, one gets

$$
\alpha=\psi \sqrt{\frac{\tilde{\kappa}}{\tilde{\kappa}+1}}\left(1-\frac{\psi^{2}}{24(\tilde{\kappa}+1)^{2}}\right)+O\left(\psi^{5}\right) .
$$



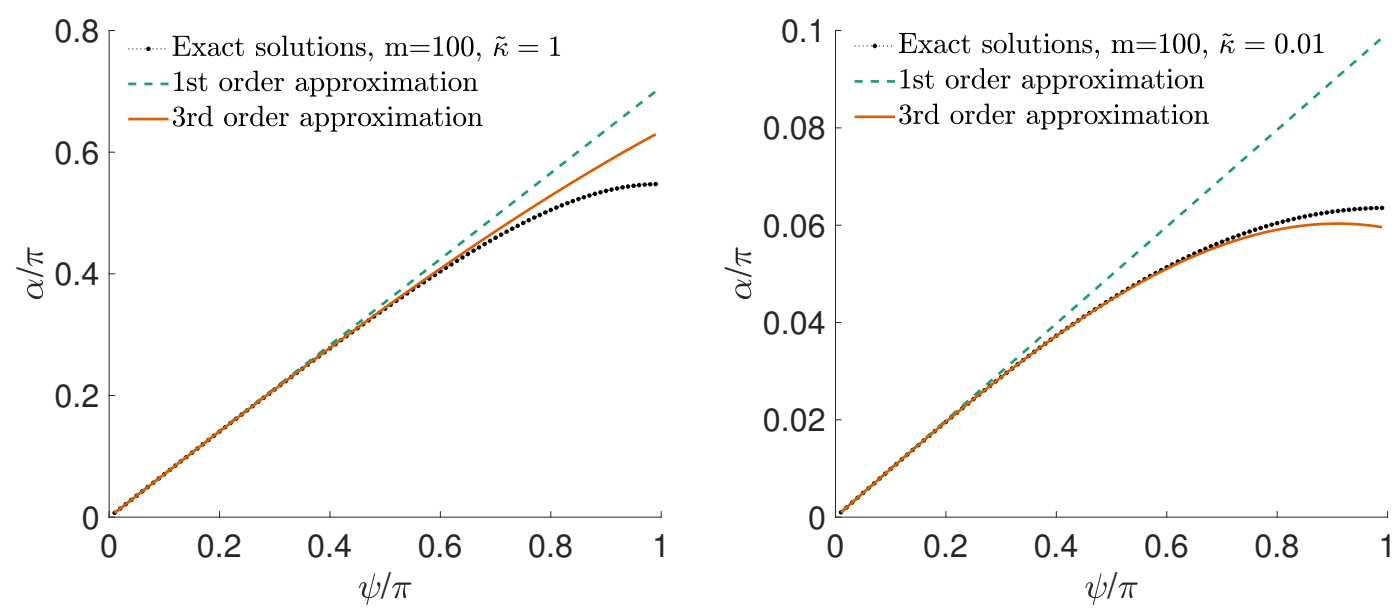

Fig. S1 The $j=0$ branch of solutions for $m=100$ compartments and its approximation by Eq. (S14). (left) $\tilde{\kappa}=1$; (right) $\tilde{\kappa}=0.01$. One can see that the first order approximation formula is more accurate when $\tilde{\kappa}$ is higher which is consistent with Eq. (S14).

Note that the third order correction is below $1 \%$ if $\psi / \pi<0.15(\tilde{\kappa}+1)$ and approximately below $10 \%$ if $\psi / \pi<0.5(\tilde{\kappa}+1)$. In particular the accuracy of the first-order approximation is always better than $10 \%$ for the first non-zero solution $\psi=\pi / m(m>1)$. This is illustrated in Fig. S1 for two values of $\tilde{\kappa}$ : 1 and 0.01. As expected, the approximation is more accurate for larger $\tilde{\kappa}$. Using this expansion we get the long-time asymptotic behavior

$$
S \approx \frac{2(1-\cos m q)}{(m q)^{2}}+A_{1}(q) \exp \left(-\frac{\pi^{2} \tilde{\kappa} t}{m^{2}(\tilde{\kappa}+1)}\right),
$$

where $A_{1}(q)$ can be read on Eq. (S9):

$$
A_{1}(q)=\frac{2 l q^{2}}{m} \frac{1+\cos m q}{(\cos q-\cos \pi / m)^{2}}\left(\frac{\cos q-\cos \alpha_{0,1}}{q^{2}-\alpha_{0,1}^{2}}\right)^{2} \beta_{0,1}^{2} .
$$

Because $\alpha_{0,1}$ is small, we have approximately

$$
A_{1}(q) \approx \frac{4(1+\cos m q)(1-\cos q)^{2}}{q^{2} m^{2}(\cos q-\cos \pi / m)^{2}},
$$

which does not depend on $\alpha_{0,1}$ anymore but only on $\psi_{0,1}=\pi / m$. In other words, $A_{1}(q)$ weakly depends on $\tilde{\kappa}$. This approximation is especially accurate at high $m$ (we checked numerically that the error is less than $3 \%$ for $m>10$, for example). This is a consequence of the remark that the global behavior of the mode, hence its norm and Fourier transform, is dictated by $\psi$ (see Eq. (31)).

From the expansion (S15) we conclude that the parameter which controls the validity of the long-time limit is not $t$ but rather $\tilde{\kappa} t /\left((\tilde{\kappa}+1) m^{2}\right)$. The $m$-dependence is obvious: $m^{2}$ is in fact the (dimensionless) time required to diffuse through all the compartments if there are no barriers. One can then see that the effect of the barriers is to increase this diffusion time by a factor $(\tilde{\kappa}+1) / \tilde{\kappa}$. In other words, the time-dependence of the signal yields an apparent diffusion coefficient

$$
D_{a p p}=D \frac{\tilde{\kappa}}{\tilde{\kappa}+1}=\frac{1}{1 / D+1 /(\kappa l)} .
$$

This formula is a well-known correction that can be derived by simple geometrical arguments [84]. When the permeability is high, the diffusion coefficient is slightly diminished. In the opposite limit $\tilde{\kappa} \ll 1$ one gets an apparent diffusion coefficient: $D_{a p p}=D \tilde{\kappa}=\kappa l$, which does not depend on the "true" diffusion 


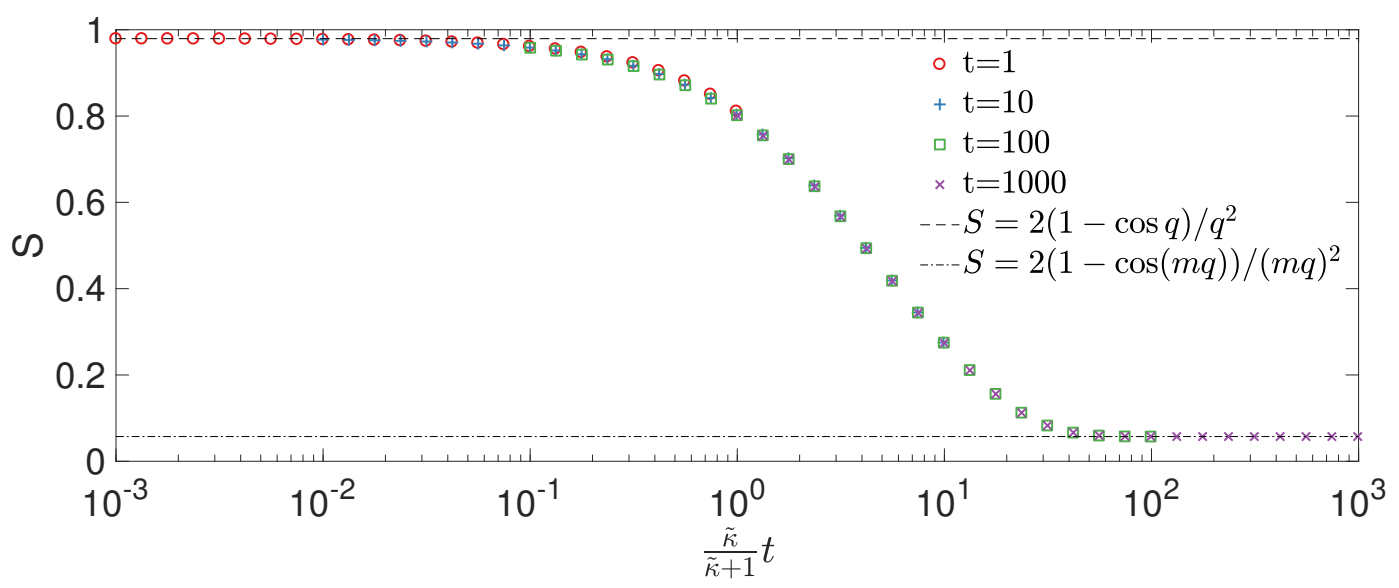

Fig. S2 Signal as a function of $\tilde{\kappa} t /(\tilde{\kappa}+1)$ at long diffusion times $(t>1)$ for $m=10$ compartments and fixed $q=0.5$. One can see that the curves fall onto one master curve. The low- and high-permeability limits (Eqs. (S17) and (S13), respectively) are plotted by dashed and dash-dotted line, respectively.

coefficient anymore. In this regime, the kinetics of diffusion are governed by the crossing of the barriers and not by the (much faster) intra-compartment diffusion.

More generally, we have:

$$
S \approx \sum_{p=0}^{m-1} A_{p}(q) \exp \left(-\frac{p^{2} \pi^{2} \tilde{\kappa} t}{m^{2}(\tilde{\kappa}+1)}\right), \quad A_{p}(q)=\left|\int_{0}^{L} u_{0, p}(x) e^{i q x} \mathrm{~d} x\right|^{2},
$$

where $A_{p}(q)$ weakly depends on $\tilde{\kappa}$. Thus in the long-time regime, the signal depends on $t$ and $\tilde{\kappa}$ via the combination $\tilde{\kappa} t /(\tilde{\kappa}+1)$.

If $1 \ll t \ll 1 / \tilde{\kappa}$, then $S \approx \sum_{p=0}^{m-1} A_{p}(q)$ and from Sec. SM. VII we get:

$$
S \approx \frac{2(1-\cos q)}{q^{2}} \quad(1 \ll t \ll 1 / \tilde{\kappa}) .
$$

The condition $t \gg 1$ means that the diffusion has averaged the magnetization inside each compartment, whereas $t \ll 1 / \tilde{\kappa}$ means that very few particles have crossed the inner barriers. As a consequence we recover the signal in the long-time limit for one compartment of length $l$ and not of length $L=m l$ (as in Eq. (S13)), even though $t \gg 1$.

Figure S2 illustrates the long-time regime $(t>1)$ for an interval segmented into $m=10$ compartments. The signal is plotted as a function of $\tilde{\kappa} t /(\tilde{\kappa}+1)$ at fixed $q=0.5$ and different times. The choice of $q$ is a compromise between the two limits given by Eqs. (S17) and (S13) (dashed and dash-dotted line, respectively). In fact, $q$ should be small enough so that the signal in the limit $\tilde{\kappa} \rightarrow 0$ is close to 1 , and large enough so that the signal in the limit $\tilde{\kappa} \rightarrow \infty$ should be close to 0 , in order to maximize the variation of the signal with $\tilde{\kappa}$. One can see that all the symbols fall onto one master curve. In particular, the transition from low- to high-permeability occurs at a fixed value of $\tilde{\kappa} t /(\tilde{\kappa}+1)$, which is around $1 / q^{2}$.

\section{SM. I.4.2 Short-time regime}

The short-time limit is the opposite case: all the branches of $j \lesssim 1 / \sqrt{t}$ have to be taken into account in the formula (S9) of the signal. However, we know that $j \pi<\alpha_{j, p}<(j+1) \pi$, so that increasing $\tilde{\kappa}$ from 0 to $\infty$ produces a net increase of the $\alpha_{j, p}$ which is less than $\pi$. As a consequence, the relative decrease of $\exp \left(-\alpha_{j, p}^{2} t\right)$ is at most $\pi^{2}(2 j+1) t \lesssim 2 \pi^{2} \sqrt{t} \ll 1$. Thus, as expected, the signal weakly depends on the permeability. As $\tilde{\kappa}$ increases the branches of solutions transform successively from the $\tilde{\kappa}=0$ limit to the $\tilde{\kappa}=\infty$ limit. Beyond $\tilde{\kappa} \sim 1 / \sqrt{t}$, the increase of $\tilde{\kappa}$ produces little change on the most contributing branches, hence on the signal. One can interpret this behavior in the following way: the dependence of 
the signal on the permeability is proportional to the fraction of particles which have reached a barrier. Indeed at short time, this fraction is given by $\sqrt{t}$. Among those particles, the ones that have crossed the barrier represent a fraction $\tilde{\kappa} t / \sqrt{t}=\tilde{\kappa} \sqrt{t}$. Hence $\tilde{\kappa} \sim 1 / \sqrt{t}$ is the value of the permeability from which almost every particle that has reached a barrier has crossed it.

\section{SM. II First exit time distribution}

\section{SM. II.1 Regular geometry}

Let us study the first exit time distribution (56) for a geometry similar to the example of Sec. 3 and SM. I: it consists of an array of $m$ identical cells of length $L / m$, where $L$ is independent of $m$, with perfectly relaxing conditions at the outer boundaries $\left(K_{ \pm}=\infty\right)$. The computations are detailed in Sec. SM. IV.5. Since $u_{n}(0)=0$, one cannot use the normalization $v(0)=1$ from Sec. 2 , so we write $u=\beta w$ with another normalization, $w^{\prime}(0)=\sqrt{\lambda / D}$, which corresponds to $\left[\begin{array}{l}a_{1}^{l} \\ b_{1}^{l}\end{array}\right]=\left[\begin{array}{l}0 \\ 1\end{array}\right]$. Because the geometry is symmetric the eigenmodes of the diffusion operator $u_{n}, n=1,2, \ldots$ are alternately symmetric or anti-symmetric (see Sec. 2.3); the latter give a zero contribution in the sum in Eqs. (55) and (56). As for the symmetric eigenmodes, one obtains:

$$
\begin{aligned}
& \int_{0}^{L} w(x) \mathrm{d} x=\frac{2 l}{\alpha} \\
& \beta^{-2}=\frac{-m l}{2} \frac{\sin \alpha\left(1+\frac{\tilde{r}}{2}\right)+\frac{\tilde{r}}{2} \alpha \cos \alpha}{\sin ^{2} \psi}\left(\sin \alpha \cos m \psi+\frac{\tilde{r} \alpha(m-1)}{m} \cos ((m-1) \psi)\right) \\
& \quad+\frac{m l}{2}\left(\frac{\sin \alpha}{\alpha}-\cos \alpha\right) \frac{\sin m \psi}{m \sin \psi},
\end{aligned}
$$

where $\alpha$ is a solution of the equation

$$
\sin \alpha \frac{\sin m \psi}{\sin \psi}+\tilde{r} \alpha \frac{\sin ((m-1) \psi)}{\sin \psi}=0 .
$$

We recall that

$$
\lambda=D \alpha^{2} / l^{2}=D \alpha^{2} m^{2} / L^{2},
$$

and we introduce the dimensionless time:

$$
t=D \tau / L^{2}
$$

Note that the solutions $\alpha$ depend only on $m$ and $\tilde{\kappa}$, hence the tail distribution is a function of $t, m, \tilde{\kappa}$, and the starting point $x$ :

$$
\begin{aligned}
& \mathbb{P}\left(T_{x}>\tau\right)=P_{x}(t, m, \tilde{\kappa}), \\
& \rho_{T_{x}}(\tau)=-\frac{\partial P_{x}}{\partial \tau}=-\frac{L^{2}}{D} \frac{\partial P_{x}}{\partial t}=\frac{L^{2}}{D} \rho_{x}(t, m, \tilde{\kappa}),
\end{aligned}
$$

$\rho_{x}(t, m, \tilde{\kappa})$ being the probability density function of the dimensionless random variable $D T_{x} / L^{2}$.

We consider now the limit $m \rightarrow \infty$. We recall that $\tilde{\kappa}=\kappa l / D=\kappa L /(m D)$, hence $\tilde{\kappa}$ depends on $m$ if $\kappa, D, L$ are fixed. However in what follows we consider $\tilde{\kappa}$ and $m$ as independent parameters. From Eq. (S21) we get that only the smallest solutions $\alpha$ contribute to the sum in Eqs. (55) and (56), hence we use Eq. (S53) which immediately implies that in the $m \rightarrow \infty$ limit all the curves fall on a unique master curve of the variable $\tilde{\kappa} t /(\tilde{\kappa}+1)$ :

$$
\begin{aligned}
& P_{x}(t, m, \tilde{\kappa}) \approx P_{x}^{*}\left(\frac{\tilde{\kappa} t}{\tilde{\kappa}+1}\right), \\
& \rho_{x}(t, m, \tilde{\kappa}) \approx \frac{\tilde{\kappa}}{\tilde{\kappa}+1} \rho_{x}^{*}\left(\frac{\tilde{\kappa} t}{\tilde{\kappa}+1}\right) .
\end{aligned}
$$




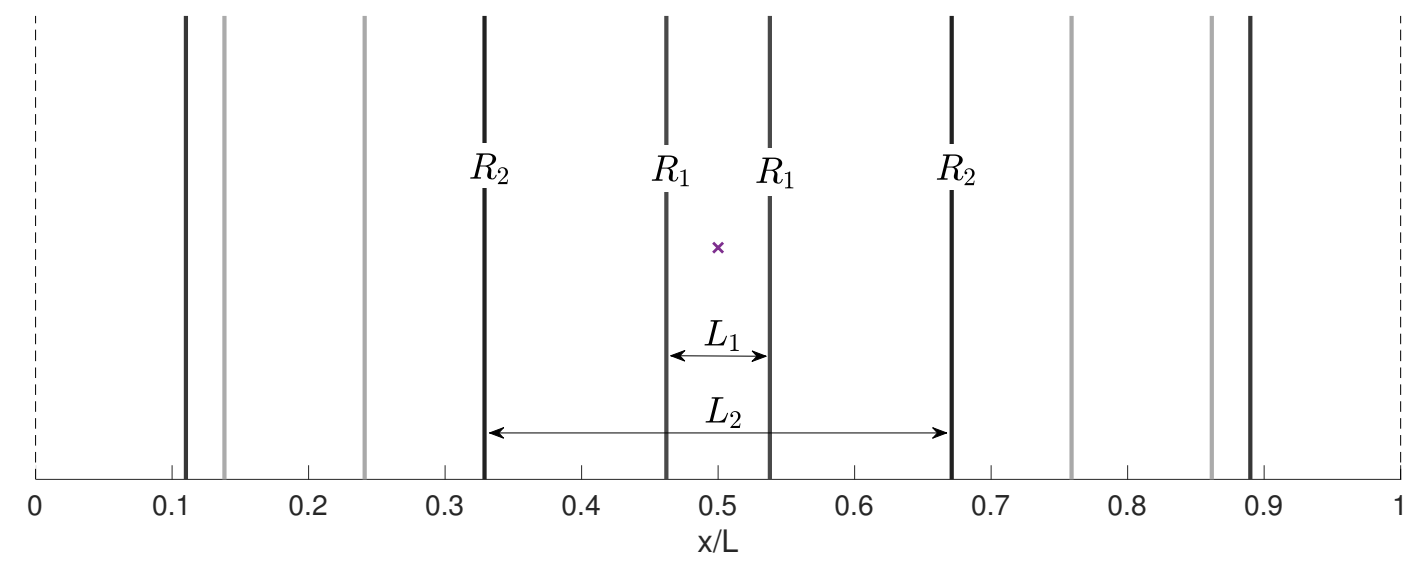

Fig. S3 An example of a random symmetric structure (with $m=11$ compartments). The solid vertical lines picture the barriers (the darker the line, the higher the resistance of the barrier). One can see this structure as nested subintervals of lengths $L_{1}<L_{2}<\ldots$ enclosed by barriers of resistances $R_{1}, R_{2}, \ldots$ The cross indicates the starting position of the particles, $x=L / 2$.

This master curve $\left(P_{x}^{*}, \rho_{x}^{*}\right)$ is precisely the one corresponding to an interval without any barriers $(\tilde{\kappa} \rightarrow \infty)$. The interpretation is that a very large number of barriers can be modeled as an effective medium with the diffusion coefficient $D_{a p p}=D \tilde{\kappa} /(\tilde{\kappa}+1)$. In particular, one obtains the formula for the mean first exit time:

$$
\mathbb{E}\left[T_{x}\right]=\frac{x(L-x)}{2 D_{a p p}}=\frac{x(L-x)}{2 D} \frac{\tilde{\kappa}+1}{\tilde{\kappa}} .
$$

Note that from the second equality in Eq. (S53) we get that one should replace $\tilde{\kappa}$ by $\tilde{\kappa}\left(1+\frac{2}{m}\right)$ in order to obtain the scaling laws (S23) and (S24), and thus Eq. (S25), to the first order in 1/m.

\section{SM. II.2 Irregular geometry}

Now we turn to an irregular geometry: the lengths of the intervals and the permeabilities of the inner barriers are randomly distributed. We still impose that the whole interval has a constant length $L$. If the number of compartments $m$ is sufficiently large, we expect that the effective medium description still holds, with an effective value of $\tilde{\kappa}$. The formula for $\tilde{\kappa}$ should involve all the lengths $l_{i}$ and permeabilities $\kappa_{i, i+1}$. Moreover in the case of a regular geometry, $l_{i}=l$ and $\kappa_{i, i+1}=\kappa$, and one should retrieve $\tilde{\kappa}=\kappa l / D$. If $l_{i}$ and $\kappa_{i, i+1}$ are independent, we find numerically that the formula

$$
\tilde{\kappa}=\frac{\langle l\rangle}{\langle r\rangle D},
$$

where $\langle\cdot\rangle$ denotes arithmetic mean, works well for large values of $m$ (typically, $m \gtrsim 100$ ). As a consequence, an irregular geometry does not differ from a regular geometry provided that the number of compartments is sufficiently large, when one replaces $l$ by $\langle l\rangle$ and $r$ by $\langle r\rangle$.

However, this formula fails at small values of $m$. The following reasoning suggests indeed that the formula of $\tilde{\kappa}$ should involve a correlation between the position of the barriers and their resistances. Let us assume for simplicity that the lengths of the compartments are randomly generated in such a way that the geometry is symmetric with respect to the middle of the interval (and that $m$ is odd). One can then see the structure as $(m-1) / 2$ nested subintervals $I_{1} \subset I_{2} \subset \cdots \subset[0, L]$ of sizes $L_{1}<L_{2}<\cdots<L$ and enclosed by barriers of resistances $R_{1}, R_{2}, \ldots, R_{(m-1) / 2}$ (see Fig. S3). We let a large number of particles diffuse from $x=L / 2$. First they diffuse inside the first subinterval $I_{1}$, so that they "feel" $\tilde{r}_{1}=D R_{1} / L L_{1}$. Let us assume that the barriers are quasi-impermeable, that is $\tilde{r}_{1} \gg 1$. According to Eq. (S25), after a time $T_{1} \sim L_{1}^{2} \tilde{r}_{1}(8 / D) \sim L_{1} R_{1}$ they have crossed the first barriers. The particle density is then quite homogeneous inside the second subinterval $I_{2}$ and so the particles feel $\tilde{r}_{2}=D R_{2} / L_{2}$. After a time 
$T_{2} \sim L_{2} R_{2}$ they cross the second barriers, they homogenize inside the third subinterval, and so on. The mean exit time is thus proportional to $\sum_{i=1}^{(m-1) / 2} R_{i} L_{i}$. According to Eq. (S25) and to the condition that we recover $\tilde{r}=r D / l$ for a regular geometry in the $m=\infty$ limit, one can guess:

$$
\tilde{\kappa}=\tilde{r}^{-1}=\frac{L^{2}}{4 D}\left(\sum_{i=1}^{(m-1) / 2} R_{i} L_{i}\right)^{-1}=\frac{L^{2}}{4 D}\left(\sum_{i=1}^{m-1} r_{i, i+1}\left|x_{i, i+1}-L / 2\right|\right)^{-1} .
$$

Interestingly, the correction $\tilde{\kappa} \rightarrow \tilde{\kappa}\left(1+\frac{2}{m}\right)$ is contained in this formula in case of a regular geometry (see Sec. SM. II.1). This formula was obtained for a symmetric geometry and it has to be refined for asymmetric geometries. In particular, it is not clear how it should be changed if the starting point $x$ is not at the middle of the interval anymore. The same reasoning suggests a formula such as:

$$
\tilde{\kappa}=\frac{x^{2}}{4 D}\left(\sum_{i=1}^{i_{0}-1} r_{i, i+1}\left(x-x_{i, i+1}\right)\right)^{-1}+\frac{(L-x)^{2}}{4 D}\left(\sum_{i=i_{0}}^{m-1} r_{i, i+1}\left(x_{i, i+1}-x\right)\right)^{-1}
$$

if $x \in \Omega_{i_{0}}$. However the numerical agreement is not as good as with a symmetric geometry and $x=L / 2$. Therefore we focus on Eq. (S27) in the following. Note that Eq. (S27) gives different weights to the barriers depending on their position with respect to the middle of the interval, which is rather intuitive. Indeed one expects a barrier located exactly at the middle of the interval to have no effect at all (given the symmetry of the geometry) whereas barriers located near the exit points should have the greatest effect.

If the permeabilities of the barriers and the lengths of the compartments are independent random variables and are distributed in a way that $\langle r\rangle$ is finite, then Eqs. (S26) and (S27) are identical in the limit $m \rightarrow \infty$. Furthermore, according to the central limit theorem we expect their deviation to be of order $m^{-1 / 2}$. Figure $\mathrm{S} 4$ shows a comparison of the two formulas. We have plotted the first exit time distribution for random structures such as the one shown in Fig. S3, with $m=11$ compartments. The lengths of the compartments and the barrier resistances follow an exponential distribution. We choose various mean values of the barrier resistances and we compute $\tilde{\kappa}$ according to Eq. (S26) or Eq. (S27). Then we apply the scaling $t \rightarrow \tilde{\kappa} t /(\tilde{\kappa}+1)$. One can see that with Eq. (S27) all the curves fall onto one master curve, whereas Eq. (S26) leads to significant deviations. Even though Eq. (S26) is less accurate than Eq. (S27), the latter involves the correlation between the position of the barriers and their permeabilities, which may be unknown in actual experiments. In this case one should use Eq. (S26), which is more "universal".

Let us conclude this section by the investigation of the particular case $\tilde{\kappa} \ll 1$. As discussed previously, in this regime the intra-compartment diffusion is much faster than the inter-compartment exchange, hence our diffusion model becomes equivalent to a random walk process on a discrete one-dimensional lattice of size $m$. The hopping rate from site $i$ to site $i+1$ and from site $i$ to site $i-1$ are respectively given by:

$$
W_{i \rightarrow i+1}=\frac{\kappa_{i, i+1}}{l_{i}}, \quad \text { and } \quad W_{i \rightarrow i-1}=\frac{\kappa_{i-1, i}}{l_{i}} .
$$

Such models of discrete random walks with random hopping rates have been considered by many authors [85-89], and in particular from the perspective of first exit times [90-95]. In particular, Murthy and Kehr discuss in [92] various cases for the distribution of the hopping rates $W_{i \rightarrow i+1}$. They consider discrete random walks starting from the left endpoint (site 0 , reflecting condition) of the lattice and analyze the first exit time through the right endpoint ( $\operatorname{site} N$, absorbing condition). By reflecting the whole lattice with respect to the left endpoint, it is equivalent to a symmetric geometry with a starting point at the middle of the interval (and $m=2 N+2$ ). In two particular cases they obtain exact formulas for the mean first exit time:

- "Symmetric case", with $W_{i \rightarrow i+1}=W_{i+1 \rightarrow i}$, which in our case corresponds to $l_{i}=l_{i+1}=l$. The mean exit time is then given by

$$
\begin{aligned}
\mathbb{E}[T] & \stackrel{M}{=} \sum_{i=1}^{N} \frac{i}{W_{m / 2+i \rightarrow m / 2+i+1}}=\frac{1}{2} \sum_{i=1}^{m-1}|i-m / 2| l r_{i, i+1} \\
= & \frac{1}{2} \sum_{i=1}^{m-1}\left|x_{i, i+1}-L / 2\right| r_{i, i+1}=\frac{L^{2}}{8 D \tilde{\kappa}} .
\end{aligned}
$$



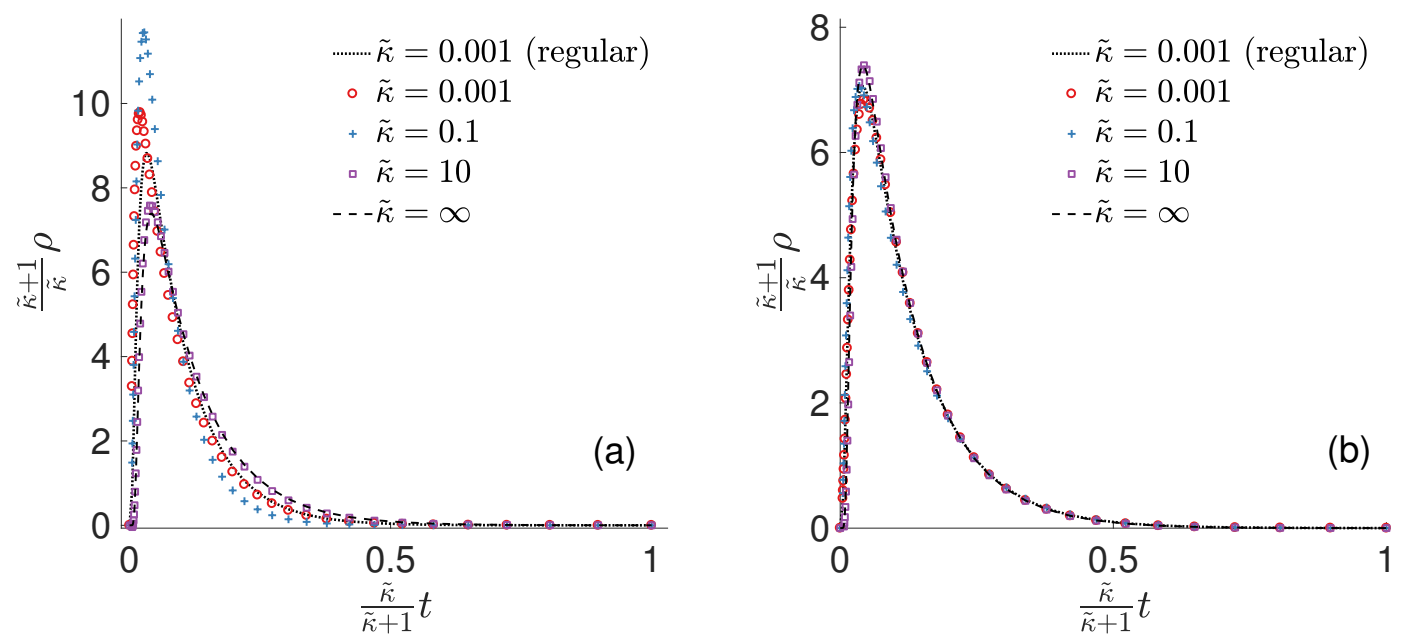

Fig. S4 The probability density of the first exit time from an interval segmented into $m=11$ compartments by random barriers of variable mean resistance (such as in Fig. S3). We apply the scale change: $t \rightarrow \tilde{\kappa} t /(\tilde{\kappa}+1)$, where $\tilde{\kappa}$ is computed either with Eq. (S26) or Eq. (S27). The dotted and dashed lines correspond to a regular geometry with quasi-impermeable and permeable barriers, respectively. (a) $\tilde{\kappa}$ is computed with Eq. (S26). One can see that the curves corresponding to the regular geometry do not coincide very well, while the curves corresponding to the random structures exhibit large deviations between each other. (b) $\tilde{\kappa}$ is computed with Eq. (S27). Visually, all the curves fall onto one master curve.

The first equality is from [92] (with suitable changes of notations). Using Eq. (S29), we obtain at the end the same formula as Eq. (S25) (recall that $\tilde{\kappa} \ll 1$ and $x=L / 2$ ), where $\tilde{\kappa}$ is given by Eq. (S27).

- "Random sojourn probabilities", with $W_{i \rightarrow i+1}=W_{i \rightarrow i-1}$, which translates into $r_{i, i+1}=r_{i-1, i}=r$.

The mean exit time is given by

$$
\begin{aligned}
\mathbb{E}[T] & \stackrel{M}{=} 89 \sum_{i=1}^{N} \frac{i}{W_{m-i \rightarrow m-i+1}}=\sum_{i=1}^{N} \sum_{k=1}^{i} l_{m-i} r=\sum_{k=1}^{N}\left(\sum_{i=1}^{k} l_{m / 2+i}\right) r \\
= & \frac{1}{2} \sum_{k=1}^{m-1}\left|x_{k, k+1}-L / 2\right| r=\frac{L^{2}}{8 D \tilde{\kappa}} .
\end{aligned}
$$

Again, the first equality is from [92]. By rearranging the sum, it transforms exactly into Eq. (S25).

We conclude that our formula Eq. (S27) introduces an effective permeability $\tilde{\kappa}$ which is consistent with the predictions of the random hopping rate models and accurately describes the first exit time distribution even for moderate number of barriers.

\section{SM. III Mathematical proofs}

In this section we prove the non-degeneracy of the eigenvalues of the diffusion operator under the assumption that all inner membranes are semi-permeable $\kappa_{i, i+1}>0, i=1, \ldots, m-1$. In fact this statement involves two facts: (i) the eigenvalues $\lambda_{n}$ of the diffusion operator are distinct; (ii) the zeros of $F$ are simple, that is $F^{\prime}\left(\lambda_{n}\right) \neq 0, n=1,2, \ldots$ (in this section, prime denotes derivative with respect to $\lambda$ ). Furthermore we shall obtain as a corollary that there are infinitely many eigenvalues $\lambda_{n}$, that they grow monotonically with the inner and outer barrier permeabilities $\kappa_{i, i+1}$ and $K_{ \pm}$, as well as a Courant nodal theorem for the eigenmodes.

The assumption of non-zero permeability is crucial. Indeed it is clear that any inner impermeable barrier would split the structure into two non-communicating parts. The eigenmodes for the whole structure would then be given by the eigenmodes for one part and the other separately. If the two parts are identical, each eigenvalue is twice degenerate. We make no other assumption about the geometry and we consider general relaxing outer boundary conditions. 
SM. III.1 Uniqueness of the eigenmodes

Let us assume that there exist two eigenmodes $u$ and $\tilde{u}$ satisfying Eqs. (4)-(9), with the same eigenvalue $\lambda$. We shall prove that $u$ is proportional to $\tilde{u}$. Because $u$ and $\tilde{u}$ both satisfy Eq. (7), one has $\frac{u^{\prime}(0)}{u(0)}=\frac{\tilde{u}^{\prime}(0)}{\tilde{u}(0)}$ hence there exists a constant $A$ such that

$$
u(0)-A \tilde{u}(0)=0 \quad \text { and } \quad u^{\prime}(0)-A \tilde{u}^{\prime}(0)=0 .
$$

Let us denote $u-A \tilde{u}$ by $w$. This function satisfies Eqs. (4)-(9) because all these equations are linear. What remains to show is that $w$ is equal to 0 over the whole interval $[0, L]$. We prove it by induction on the index of the compartment $i$. The main mathematical argument is Cauchy-Lipschitz uniqueness theorem for second order linear differential equations (U): "if $f$ satisfies a second order linear differential equation over an interval $\Omega$ and $f(c)=f^{\prime}(c)=0$, with $c \in \Omega$, then $f(x)=0$ for every $x \in \Omega$ ".

- We apply (U) to $\left.w\right|_{\Omega_{1}}:\left.w\right|_{\Omega_{1}}(0)=\left.w\right|_{\Omega_{1}} ^{\prime}(0)=0$ and $\left.D_{1} w^{\prime \prime}\right|_{\Omega_{1}}+\left.\lambda w\right|_{\Omega_{1}}=0$, hence $\left.w\right|_{\Omega_{1}}=0$.

- Let us assume that $\left.w\right|_{\Omega_{i}}=0$, with $0<i<m-1$. Then, because $\kappa_{i, i+1} \neq 0$, the inner boundary conditions in Eqs. (5) and (6) imply that $\left.w\right|_{\Omega_{i+1}}\left(x_{i, i+1}\right)=\left.w^{\prime}\right|_{\Omega_{i+1}}\left(x_{i, i+1}\right)=0$. Because $\left.w\right|_{\Omega_{i+1}}$ obeys the equation $\left.D_{i+1} w^{\prime \prime}\right|_{\Omega_{i+1}}+\left.\lambda w\right|_{\Omega_{i+1}}=0$, one can apply again (U), which implies $\left.w\right|_{\Omega_{i+1}}=0$.

SM. III.2 Simplicity of the zeros of $F$

Now we prove that $F^{\prime}\left(\lambda_{n}\right) \neq 0$ for any eigenvalue $\lambda_{n}$. In order to simplify the notations we consider the case where $K_{ \pm}$are finite. However the proof follows the same steps in the case of infinite $K_{ \pm}$. Throughout the proof we implicitly discard the case $\lambda=0$. Let us recall that if we consider the function $v(\lambda, x)$ which satisfies Eqs. (4)-(7) as well as the condition $v(0)=1$ (we have proven above that this function is unique), then

$$
F(\lambda)=\frac{K_{+}}{D_{m}} v(\lambda, L)+\frac{\partial v}{\partial x}(\lambda, L)
$$

Instead of writing $v$ as a sum of sine and cosine functions (see Eq. (11)), we introduce an amplitude and phase representation:

$$
\left.v\right|_{\Omega_{i}}(x)=A_{i}(\lambda) \cos \left(\sqrt{\lambda / D_{i}} x+\phi_{i}(\lambda)\right)=A_{i}(\lambda) \cos \left(\Phi_{i}(\lambda, x)\right),
$$

with $A_{i} \geq 0$. It is clear from Eq. (11) that $A_{i}$ and $\phi_{i}$ do not depend on $x$. Moreover we have proven in the above paragraph that $A_{i}(\lambda)$ is non-zero for all $i$ and $\lambda$. We now translate the boundary conditions (5)-(8) in terms of $\Phi_{i}$. Equation (7) yields: $K_{-} A_{i} \cos \phi_{1}+\sqrt{\lambda D_{1}} \sin \phi_{1}=0$, hence

$$
\tan \phi_{1}=-\frac{K_{-}}{\sqrt{\lambda D_{1}}} \quad\left(-\pi / 2 \leq \phi_{1} \leq 0\right) .
$$

Equtaions (5) and (6) can be restated as

$$
-A_{i} \sqrt{\lambda D_{i}} \sin \left(\Phi_{i}\right)=-A_{i+1} \sqrt{\lambda D_{i+1}} \sin \left(\Phi_{i+1}\right)=\kappa_{i, i+1}\left(A_{i+1} \cos \left(\Phi_{i+1}\right)-A_{i} \cos \left(\Phi_{i}\right)\right)
$$

at $x=x_{i, i+1}$, hence by eliminating $A_{i}$ and $A_{i+1}$, we get

$$
\frac{\cot \Phi_{i}\left(\lambda, x_{i, i+1}\right)}{\sqrt{D_{i}}}-\frac{\cot \Phi_{i+1}\left(\lambda, x_{i, i+1}\right)}{\sqrt{D_{i+1}}}=r_{i, i+1} \sqrt{\lambda},
$$

with $0 \leq \Phi_{i+1}\left(\lambda, x_{i, i+1}\right)-\Phi_{i}\left(\lambda, x_{i, i+1}\right)<\pi$. Finally, one can rewrite Eq. (S30) as

$$
\begin{aligned}
F(\lambda) & =A_{m}(\lambda)\left(\frac{K_{+}}{D_{m}} \cos \Phi_{m}(\lambda, L)-\sqrt{\lambda / D_{m}} \sin \Phi_{m}(\lambda, L)\right) \\
& =A_{m+1}(\lambda) \cos \left(\Phi_{m}(\lambda, L)+\phi_{m+1}(\lambda)\right),
\end{aligned}
$$


with:

$$
A_{m+1}(\lambda)=A_{m}(\lambda) \sqrt{\left(\frac{K_{+}}{D_{m}}\right)^{2}+\frac{\lambda}{D_{m}}}, \quad \cot \phi_{m+1}(\lambda)=\frac{K_{+}}{\sqrt{\lambda D_{m}}}
$$

and $0 \leq \phi_{m+1} \leq \pi / 2$. We have $A_{m+1}(\lambda) \neq 0$ for any $\lambda$ and $-\pi / 2<\Phi_{m}(0, L)+\phi_{m+1}(0) \leq \pi / 2$, hence Eq. (22) is equivalent to $\Phi_{m}\left(\lambda_{n}, L\right)+\phi_{m+1}\left(\lambda_{n}\right)=(2 n-1) \pi / 2$. The derivative of $F$ at $\lambda=\lambda_{n}$ is then given by

$$
F^{\prime}\left(\lambda_{n}\right)=(-1)^{n} A_{m+1}\left(\lambda_{n}\right)\left(\Phi_{m}^{\prime}\left(\lambda_{n}, L\right)+\phi_{m+1}^{\prime}\left(\lambda_{n}\right)\right)
$$

It is clear from Eq. (S35) that $\phi_{m+1}^{\prime}(\lambda) \geq 0$ for any $\lambda$. In order to prove that $F^{\prime}\left(\lambda_{n}\right) \neq 0$, it is then sufficient to show that $\Phi_{m}^{\prime}(\lambda, L)>0$. We prove by induction on the index of the compartment $i$ that $\Phi_{i}^{\prime}(\lambda, x)$ is positive for any $\lambda$ and any $x \in \Omega_{i}$ :

- From Eq. (S32) we get that $\phi_{1}$ is an increasing function of $\lambda$. As $\Phi_{1}(\lambda, x)=\sqrt{\lambda / D_{1}} x+\phi_{1}(\lambda)$, we immediately get that $\Phi_{1}^{\prime}(\lambda, x)>0$ for any $x \in \Omega_{1}$.

- Let us assume that $\Phi_{i}\left(\lambda, x_{i, i+1}\right)$ is an increasing function of $\lambda$. According to Eq. (S33), let us introduce the function:

$$
f(\lambda, y)=\cot ^{-1}\left(\sqrt{\frac{D_{i+1}}{D_{i}}} \cot y-r_{i, i+1} \sqrt{\lambda D_{i+1}}\right) .
$$

Because cot is a decreasing function, $f$ is an increasing function of $y$ and a non-decreasing function of $\lambda$, which implies that $\Phi_{i+1}\left(\lambda, x_{i, i+1}\right)=f\left(\lambda, \Phi_{i}\left(\lambda, x_{i, i+1}\right)\right)$ is an increasing function of $\lambda$. It is then clear that $\Phi_{i+1}(\lambda, x)=\Phi_{i+1}\left(\lambda, x_{i, i+1}\right)+\sqrt{\lambda / D_{i+1}}\left(x-x_{i, i+1}\right)$ is an increasing function of $\lambda$ for any $x \in \Omega_{i+1}$.

This proves the simplicity of the zeros of $F$. Moreover, we also obtain that $\Phi_{m}(\lambda, L)$ grows indefinitely with $\lambda$. According to Eq. (S34), this implies that there are infinitely many values of $\lambda$ such that $F(\lambda)=0$. In other words, there are infinitely many eigenvalues $\lambda_{n}$.

SM. III.3 Monotonicity of the eigenvalues with respect to the permeabilities

The previous computations enable us to show that the eigenvalues grow monotonically with the inner and outer permeabilities $\kappa_{i, i+1}$ and $K_{ \pm}$. In fact, because $\Phi_{m}(\lambda, L)+\phi_{m+1}(\lambda)$ is an increasing function of $\lambda$, we just have to prove that $\Phi_{m}(\lambda, L)+\phi_{m+1}(\lambda)$ is a non-increasing function of $\kappa_{i, i+1}$ and $K_{ \pm}$, which follows immediately from Eqs. (S32), (S37) and (S35).

\section{SM. III.4 Courant nodal theorem}

Let us define the nodal domains of an eigenmode $u_{n}$ as connected components on which $u_{n}$ does not change sign. We prove here that $u_{n}$ has exactly $n$ nodal domains, which means that it changes sign $n-1$ times (recall that we numbered the modes $n=1,2, \ldots$ ). Note that these sign changes can occur at discontinuity points of $u_{n}$. The proof relies on the amplitude and phase representation detailed above. Let us then write

$$
u_{n}(x)=A\left(\lambda_{n}, x\right) \cos \left(\Phi\left(\lambda_{n}, x\right)\right),
$$

where $A$ and $\Phi$ are piecewise continuous functions of $x$ defined by $\left.A\right|_{\Omega_{i}}=A_{i}$ and $\left.\Phi\right|_{\Omega_{i}}=\Phi_{i}$. The changes of sign of the eigenmode occur when the phase $\Phi$ crosses an odd multiple of $\pi / 2$. Indeed, $A\left(\lambda_{n}, x\right)$ has a constant sign, and from Eq. (S33) we get that the jumps of $\Phi$ at the barriers are always less than $\pi$ (which means that $\Phi$ cannot cross two odd multiples of $\pi / 2$ at the same time).

Moreover, we know the phase at the left endpoint: $\Phi\left(\lambda_{n}, 0\right)=\phi_{1}\left(\lambda_{n}\right) \in[-\pi / 2 ; 0]$ and the phase at the right endpoint: $\Phi\left(\lambda_{n}, L\right)=(2 n-1) \pi / 2-\phi_{m+1}\left(\lambda_{n}\right) \in[(n-1) \pi ;(n-1) \pi+\pi / 2]$. We conclude that the interval $\left(\Phi\left(\lambda_{n}, 0\right) ; \Phi\left(\lambda_{n}, L\right)\right)$ contains exactly $n-1$ odd multiple of $\pi / 2$, thus the eigenmode has $n$ nodal domains. 
SM. IV Computations for an array of identical cells with symmetric relaxation conditions at the outer boundaries

In this section we extend the computation presented in Sec. 3.1 by allowing relaxation or leakage at the endpoints of the interval. In other words, we relax the reflecting boundary conditions $K_{ \pm}=0$ at the outer membranes. In particular we will also study the limit $K_{ \pm} \rightarrow \infty$ which is the perfectly relaxing case that we use in Sec. SM. II. The cells are the same: $l_{i}=l, D_{i}=D, \kappa_{i, i+1}=\kappa$, and the relaxation coefficients are identical: $K_{+}=K_{-}=K$. In addition to the notations (41), we introduce: $\tilde{K}=K l / D$.

\section{SM. IV.1 Eigenmodes}

Because the geometry is symmetric we know that $\epsilon= \pm 1$. In this case we need to solve the general equation (43)

$$
\mathcal{K}^{-1} \mathcal{M}^{m}\left[\begin{array}{c}
\alpha \\
\tilde{K}
\end{array}\right]=\epsilon\left[\begin{array}{c}
\alpha \\
-\tilde{K}
\end{array}\right]
$$

With the help of Eq. (45) we can compute the matrix $\mathcal{K}^{-1} \mathcal{M}^{m}$ :

$$
\mathcal{K}^{-1} \mathcal{M}^{m}=\left[\begin{array}{cc}
\cos \alpha \frac{\sin m \psi}{\sin \psi}-\frac{\sin (m-1) \psi}{\sin \psi} & \sin \alpha \frac{\sin m \psi}{\sin \psi}+\tilde{r} \alpha \frac{\sin (m-1) \psi}{\sin \psi} \\
-\sin \alpha \frac{\sin m \psi}{\sin \psi} & \cos \alpha \frac{\sin m \psi}{\sin \psi}-\frac{\sin (m-1) \psi}{\sin \psi}
\end{array}\right] .
$$

Thus Eq. (S39) yields the system

$$
\left\{\begin{array}{l}
\left(\cos \alpha+\tilde{K} \frac{\sin \alpha}{\alpha}\right) \frac{\sin m \psi}{\sin \psi}-(1-\tilde{r} \tilde{K}) \frac{\sin (m-1) \psi}{\sin \psi}= \pm 1 \\
\left(\cos \alpha-\frac{1}{\tilde{K}} \alpha \sin \alpha\right) \frac{\sin m \psi}{\sin \psi}-\frac{\sin (m-1) \psi}{\sin \psi}=\mp 1
\end{array}\right.
$$

which is equivalent to the equation

$$
\left(\cos \alpha+\frac{1}{2}\left(\frac{\tilde{K}}{\alpha}-\frac{\alpha}{\tilde{K}}\right) \sin \alpha\right) \frac{\sin m \psi}{\sin \psi}-\left(1-\frac{\tilde{r} \tilde{K}}{2}\right) \frac{\sin (m-1) \psi}{\sin \psi}=0 .
$$

Combined with Eq. (44) it forms a system whose solutions $\alpha_{n}$ determine the eigenvalues $\lambda_{n}$. Compared to the $K=0$ case from Sec. 3.1, the solutions $\alpha_{n}$ are modified and in general increase with $\tilde{K}$.

In the particular case $\tilde{K}=2 \tilde{\kappa}$, Eq. (S42) simplifies into

$$
\frac{\sin m \psi}{\sin \psi}=0 \quad \text { or } \quad \cos \alpha+\frac{1}{2}\left(\frac{\tilde{K}}{\alpha}-\frac{\alpha}{\tilde{K}}\right) \sin \alpha=0 .
$$

The first equation gives the $\alpha_{j, p}(p=1, \ldots, m-1)$ from the earlier considered $K=0$ case. The second equation gives the solutions of $\cos \psi= \pm 1$ that are not multiple of $\pi$ (that we denote as $\alpha_{j, m}$ if $j$ is even and $\alpha_{j, 0}$ if $j$ is odd, to be consistent with our previous notations). The condition $\tilde{K}=2 \tilde{\kappa}$ can be interpreted as "one inner barrier is equivalent to two stacked outer barriers" or equivalently "the crossing of one inner barrier transforms $\left[\begin{array}{c}-1 \\ \frac{\tilde{K}}{\alpha}\end{array}\right]$ into $\left[\begin{array}{c}1 \\ \frac{\tilde{K}}{\alpha}\end{array}\right]$ ". In this way the reason why the $\alpha_{j, p}$ are solutions becomes clear: the matrix $\mathcal{K}\left(\mathcal{K}^{-1} \mathcal{M}^{m}\right)=\mathcal{M}^{m}$ should send $\left[\begin{array}{c}1 \\ \frac{\tilde{K}}{\alpha}\end{array}\right]$ onto plus or minus itself. The $\alpha_{j, p}$ (with $1<p<m$ ) are solutions of $\mathcal{M}^{m}= \pm \mathcal{I}_{2}$ and the $\alpha_{j, 0}$ and $\alpha_{j, m}$ are such that $\left[\begin{array}{c}1 \\ \frac{\tilde{K}}{\alpha}\end{array}\right]$ is an eigenvector of $\mathcal{M}$.

As a consequence, the spectrum for the case $\tilde{K}=2 \tilde{\kappa}$ differs little from the spectrum for the impermeable outer boundary condition. The only difference lies in the beginning and the end of the branches (see Fig. S5). This is nevertheless not a small difference because the eigenvalue $\lambda=0$ (which is absent of the spectrum if $\tilde{K}>0$ ) plays an important role in the long-time limit of the diffusion propagator as we have discussed in Sec. SM. I.4.1. 


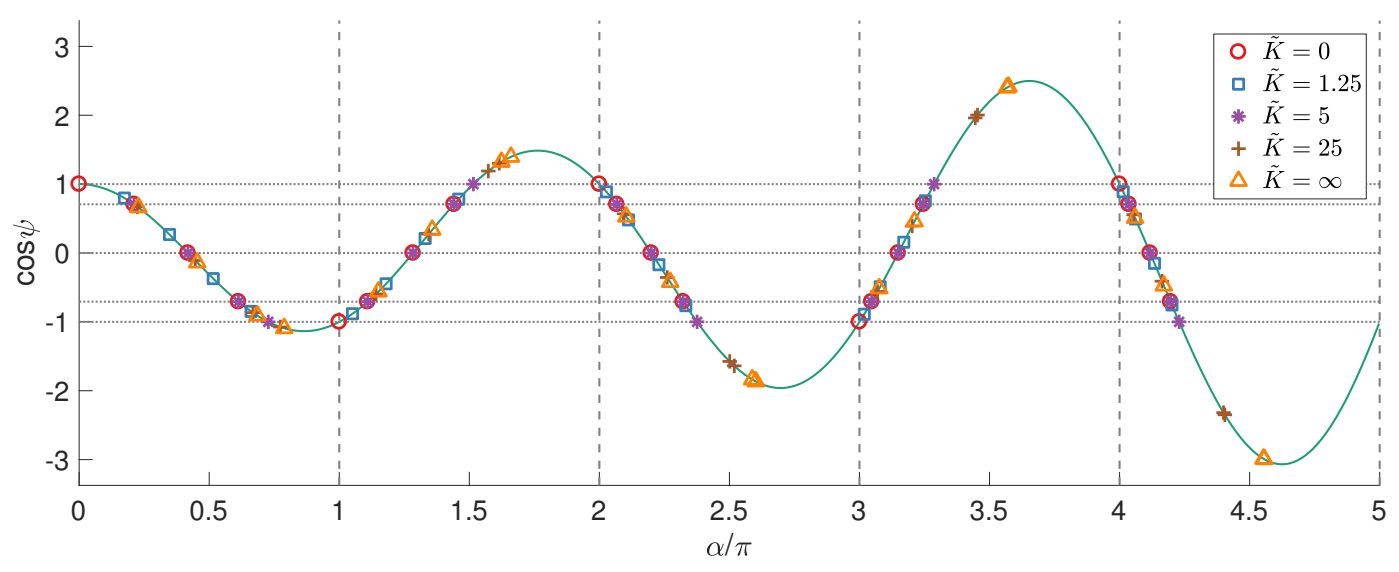

Fig. S5 Spectrum of the finite periodic geometry with $m=4$ compartments and $\tilde{r}=0.4$, for $\tilde{K}=0$ (circles), $\tilde{K}=$ $\tilde{\kappa} / 2=1.25$ (squares), $\tilde{K}=2 \tilde{\kappa}=5$ (asterisks), $\tilde{K}=10 \tilde{\kappa}=25$ (pluses) and $\tilde{K}=\infty$ (triangles). The values of $\alpha$ increase with $\tilde{K}$. Notice how the spectra for $\tilde{K}=0$ and $\tilde{K}=2 \tilde{\kappa}$ coincide except at the beginning and the end of the branches.

Beyond this special value of $\tilde{K}$, the solutions $\alpha_{n}$ continue to increase so that some values of $\psi_{n}$ become complex (because $|\cos \psi|>1$, which is apparent in Fig. S5). More precisely they have the general form $\psi=i x$ or $\psi=\pi+i x$, with $x \in \mathbb{R}$. These values correspond to eigenmodes strongly localized inside the outer compartments. Indeed, Eq. (31) implies that the coefficients $a$ and $b$ vary like linear combinations of cosh and sinh functions of the compartment index $i$. The physical interpretation is simple: when $\tilde{K} \gg \tilde{\kappa}$ we are indeed in a regime where the leakage through the outer membranes is much faster than the exchange through the inner barriers. As a consequence the outer compartments evolve separately from the inner compartments, which corresponds mathematically to the existence of localized eigenmodes. On the other hand, when $\tilde{K} \ll \tilde{\kappa}$, the outer leakage is much slower than the inner exchange, thus all compartments are coupled. We treat the limit $\tilde{K} \rightarrow \infty$ below in Sec. SM. IV.5.

\section{SM. IV.2 Computation of the norm}

The general formula (24) reads

$$
\beta^{-2}=\frac{l}{2}\left|\frac{\mathrm{d}}{\mathrm{d} \alpha}\left(\left[\begin{array}{ll}
\frac{\tilde{K}}{\alpha} & 1
\end{array}\right] \mathcal{T}(\alpha)\left[\begin{array}{c}
1 \\
\frac{\tilde{K}}{\alpha}
\end{array}\right]\right)\right|_{\alpha=\alpha_{n}} .
$$

After lengthy computations, one gets

$$
\begin{aligned}
\beta^{-2} & =\frac{\epsilon l}{2} \frac{\sin \alpha\left(1+\frac{\tilde{r}}{2}\right)+\frac{\tilde{r}}{2} \alpha \cos \alpha}{\sin ^{2} \psi}\left(\left(\frac{\tilde{K}}{\alpha}\right)^{2} \sin \alpha+2 \frac{\tilde{K}}{\alpha} \cos \alpha-\sin \alpha\right)\left(\frac{(m-1) \sin \psi}{\sin (m-1) \psi}-\cos m \psi\right) \\
& +\frac{\epsilon l}{2} \frac{\sin m \psi}{\sin \psi}\left(\frac{\sin \alpha}{\alpha}\left(1+2 \tilde{K}+\left(\frac{\tilde{K}}{\alpha}\right)^{2}\right)+\left(1-\left(\frac{\tilde{K}}{\alpha}\right)^{2}\right) \cos \alpha\right) .
\end{aligned}
$$

Note that when $\tilde{K}=2 \tilde{\kappa}$ we have to compute separately the cases $\psi=0$ and $\psi=\pi$. We get

$$
\begin{array}{lc}
\beta^{-2}=\frac{m l}{2}\left(-\cos \alpha-2 \frac{\tilde{K}}{\alpha} \sin \alpha+\left(\frac{\tilde{K}}{\alpha}\right)^{2}\left(\cos \alpha+\frac{m-1}{m \tilde{\kappa}}\right)+2 \frac{\tilde{K}}{m \alpha^{2}}\right) & \text { if } \psi=0, \\
\beta^{-2}=\frac{m l}{2}\left(\cos \alpha+2 \frac{\tilde{K}}{\alpha} \sin \alpha-\left(\frac{\tilde{K}}{\alpha}\right)^{2}\left(\cos \alpha-\frac{m-1}{m \tilde{\kappa}}\right)+2 \frac{\tilde{K}}{m \alpha^{2}}\right) & \text { if } \psi=\pi .
\end{array}
$$


SM. IV.3 Fourier transform

Except for the conditions at the outer boundaries, the geometry is the same as in Sec. SM. I.2. Hence the computation follows the same steps. Using the condition (S39), we are led to compute the product

$$
\mathcal{L}\left(\mathcal{I}_{2}-e^{i q} \mathcal{R}^{-1} \mathcal{K}^{-1}\right)\left(\mathcal{I}_{2}-\epsilon e^{i m q} \mathcal{K} \mathcal{S}\right)\left[\begin{array}{c}
\alpha \\
\tilde{K}
\end{array}\right]
$$

Skipping the technical computations, one gets depending on $\epsilon= \pm 1$

$$
\begin{array}{ll}
\int_{0}^{L} v(x) e^{i \gamma g \delta x} \mathrm{~d} x=\frac{e^{i m q / 2} 2 l}{\left(q^{2}-\alpha\right)^{2}(\cos q-\cos \psi)}(A \cos (m q / 2)+B \sin (m q / 2)) & \text { if } \epsilon=+1, \\
\int_{0}^{L} v(x) e^{i \gamma g \delta x} \mathrm{~d} x=\frac{-i e^{i m q / 2} 2 l}{\left(q^{2}-\alpha\right)^{2}(\cos q-\cos \psi)}(A \sin (m q / 2)-B \cos (m q / 2)) & \text { if } \epsilon=-1,
\end{array}
$$

where

$$
A=\tilde{K}\left((\cos \alpha-\cos q)+\frac{\tilde{r}}{2}(q \sin q-\alpha \sin \alpha)\right), \quad B=q(\cos q-\cos \alpha)\left(1-\frac{\tilde{r} \tilde{K}}{2}\right)
$$

\section{SM. IV.4 Complete expression of the dMRI signal}

According to Eq. (S4), the signal is expressed as a sum over all eigenmodes $u_{n}$. We recall that the eigenmodes are alternately symmetric ( odd $n$ ) and anti-symmetric (even $n$ ). Combining the above results (S45)-(S48), one gets

$$
S=\sum_{n=1}^{\infty} \frac{\left(A_{n}^{2}+B_{n}^{2}+(-1)^{n-1}\left(A_{n}^{2}-B_{n}^{2}\right) \cos m q+(-1)^{n-1} 2 A_{n} B_{n} \sin m q\right) 4 \beta_{n}^{2} e^{-\alpha_{n}^{2} t}}{\left(q^{2}-\alpha_{n}^{2}\right)^{2}\left(\cos q-\cos \psi_{n}\right)^{2}}
$$

where $\beta_{n}$ is given by Eq. (S45), $A_{n}$ and $B_{n}$ by Eq. (S48), $\psi_{n}$ by Eq. (44) and $\alpha_{n}$ are solutions of Eq. (S42). For $m=1$, we recover the signal derived by Coy and Callaghan [66].

SM. IV.5 Perfectly relaxing outer boundaries

Note that the limit $\tilde{K} \rightarrow \infty$ is singular because of the chosen normalization (10). This is particularly clear in Eq. (18) where $b_{1}^{l} \rightarrow \infty$. In fact, $\tilde{K}=\infty$ represents Dirichlet conditions at the outer boundaries: $u(0)=u(L)=0$. To avoid the singularity we use another normalization:

$$
u=\beta w, \quad w^{\prime}(0)=\sqrt{\lambda / D},
$$

which corresponds to the coefficients (for $w$ )

$$
\left[\begin{array}{l}
a_{1}^{l} \\
b_{1}^{l}
\end{array}\right]=\left[\begin{array}{c}
\frac{\sqrt{\lambda D_{1}}}{K_{-}} \\
1
\end{array}\right]
$$

SM. IV.5.1 Study of the spectrum

When $\tilde{K} \rightarrow \infty$, Eq. (S42) simplifies into

$$
\sin \alpha \frac{\sin m \psi}{\sin \psi}+\tilde{r} \alpha \frac{\sin (m-1) \psi}{\sin \psi}=0 .
$$

We now study the solutions of this equation in three different regimes: high-permeability, low-permeability, and very large number of compartments. We rely on the discussion developed in Sec. 2.5, which leads us to the following conclusions. 
High-permeability regime In the high-permeability regime $(\tilde{r} \ll 1)$, the solutions are located near the limits $\alpha_{0}=n \pi / m$, which correspond also to $\psi_{0}=n \pi / m(n=1,2, \ldots)$. More precisely one can compute the first-order expansion:

$$
\begin{cases}\alpha_{n} \approx \frac{n \pi}{m}\left(1-\frac{\tilde{r}(m-2)}{2 m}\right) & \text { if } n \text { is not a multiple of } m \\ \alpha_{n} \approx \frac{n \pi}{m}\left(1-\frac{\tilde{r}(m-1)}{m}\right) & \text { otherwise. }\end{cases}
$$

As already noted this case presents no difficulty from the numerical point of view.

Low-permeability regime In the low-permeability regime $(\tilde{\kappa} \ll 1)$, the solutions are divided into two categories.

- First, the solutions corresponding to the "inner" compartments: $1<k<m$. These solutions form groups located around $\alpha_{0}=j \pi$ ( $j$ being an integer). In fact they correspond to $\psi \in \mathbb{R}$, at which $\sin (m \psi)$ and $\sin ((m-1) \psi)$ are of the same order. This implies that Eq. (S20) becomes in the low-permeability limit

$$
\frac{\sin ((m-1) \psi)}{\sin \psi}=0
$$

which is (almost) the equation of the spectrum of $m-1$ identical cells with impermeable outer boundaries (47). One gets simply the solutions $\psi_{0}=p \pi /(m-1), p=1, \ldots, m-2$, thus the solutions in the first category are approximately determined by

$$
\cos \alpha-\frac{\tilde{r}}{2} \alpha \sin \alpha=\cos (p \pi /(m-1)), \quad p=1, \ldots, m-2 .
$$

We study this equation in details in Sec. SM. VIII. In particular, applying Eq. (S87) one gets for the $m-2$ first solutions:

$$
\alpha_{n} \approx 2 \sqrt{\tilde{\kappa}} \sin \left(\frac{n \pi}{2(m-1)}\right), \quad n=1, \ldots, m-2 .
$$

- Second, the solutions corresponding to the outer compartments $k=1, m$. These solutions form pairs $\alpha_{ \pm}$such that

$$
\begin{aligned}
\left(n+\frac{1}{2}\right) \pi-\alpha_{+} & \approx\left(n+\frac{1}{2}\right) \pi-\alpha_{-} \sim \frac{\tilde{\kappa}}{(n+1 / 2) \pi}, \\
\alpha_{+}-\alpha_{-} & \sim\left(\frac{\tilde{\kappa}}{(n+1 / 2) \pi}\right)^{m-1},
\end{aligned}
$$

with $n=1,2, \ldots$. Therefore in the low-permeability limit $(\tilde{\kappa} \rightarrow 0)$ these pairs are very difficult to detect, especially when one is dealing with a large number of compartments $m$. As explained in Sec. 4.1, even if one finds the roots, the subsequent computation of the eigenmodes and their norm may be inaccurate. However in this regime these solutions are much larger than the smallest one from the first category which go to zero according to Eq. (S52). Hence they have little influence on the first exit time distribution (56) because of the very fast exponential decay compared to the first terms of the sum.

Limit $m \rightarrow \infty$ From the above discussion we get that the $m-2$ first solutions of Eq. (S20), $\alpha_{1}, \ldots, \alpha_{m-2}$, satisfy

$$
n \pi / m<\psi_{n}<n \pi /(m-1), \quad n=1, \ldots, m-2 .
$$

Thus one may write $\psi_{n}=\frac{n \pi}{m-x}$, with $0<x<1$. Let us rewrite Eq. (S20) as

$$
\sin \alpha_{n} \sin \left(m \psi_{n}\right)+\tilde{r} \alpha_{n} \sin \left((m-1) \psi_{n}\right)=(-1)^{n}\left(\sin \alpha_{n} \sin \left(\frac{x n \pi}{m-x}\right)-\tilde{r} \alpha_{n} \sin \left(\frac{(1-x) n \pi}{m-x}\right)\right)=0
$$

Now we study the limit $m \rightarrow \infty$ with fixed $n$. Then $\psi_{n}, \alpha_{n} \ll 1$ and the above equation transforms into

$$
\frac{(-1)^{n} \alpha_{n} n \pi}{m-x}(x-\tilde{r}(1-x))=0
$$


from which we get $x=\tilde{r} /(1+\tilde{r})=1 /(1+\tilde{\kappa})$. Let us use the expansion (S14):

$$
\alpha_{n} \approx \sqrt{\frac{\tilde{\kappa}}{\tilde{\kappa}+1}} \frac{n \pi}{m-\frac{1}{\tilde{\kappa}+1}} \approx \sqrt{\frac{\tilde{\kappa}\left(1+\frac{2}{m}\right)}{\tilde{\kappa}\left(1+\frac{2}{m}\right)+1}} \frac{n \pi}{m}, \quad n=1, \ldots, m-2 .
$$

\section{SM. IV.5.2 Computation of the norm}

The formula (24) for the norm becomes

$$
\beta^{-2}=\int_{0}^{L} w^{2}=\left.\frac{-\sqrt{D_{1}}}{2 \eta} \frac{\mathrm{d}}{\mathrm{d} \sqrt{s}}\left(\left[\frac{K_{+}}{K_{-}} \frac{\sqrt{D_{m} s}}{K_{-}}\right] \mathcal{T}(s)\left[\begin{array}{c}
\frac{\sqrt{D_{1} s}}{K_{-}} \\
1
\end{array}\right]\right)\right|_{s=\lambda} .
$$

In the particular geometry we are dealing with and in the case $\tilde{K}=\infty$, this gives

$$
\begin{aligned}
\beta^{-2} & =\frac{-\epsilon l}{2}\left|\left[\begin{array}{ll}
1 & 0
\end{array}\right] \frac{\mathrm{d} \mathcal{T}}{\mathrm{d} \alpha}\left[\begin{array}{l}
0 \\
1
\end{array}\right]\right| \\
& =\frac{-\epsilon m l}{2} \frac{\sin \alpha\left(1+\frac{\tilde{r}}{2}\right)+\frac{\tilde{r}}{2} \alpha \cos \alpha}{\sin ^{2} \psi}\left[\sin \alpha \cos m \psi+\frac{\tilde{r} \alpha(m-1)}{m} \cos ((m-1) \psi)\right] \\
& +\frac{\epsilon m l}{2}\left(\frac{\sin \alpha}{\alpha}-\cos \alpha\right) \frac{\sin m \psi}{m \sin \psi} .
\end{aligned}
$$

\section{SM. IV.6 Computation of the Fourier transform}

In the same way, the computation of the Fourier transform of $w$ simplifies into

$$
\frac{e^{i m q / 2} 2 l \alpha}{\left(q^{2}-\alpha\right)^{2}(\cos q-\cos \psi)} \times\left\{\begin{array}{ll}
A \cos (m q / 2)+B \sin (m q / 2) & \text { if } \epsilon=+1 \\
-i(A \sin (m q / 2)-B \cos (m q / 2)) & \text { if } \epsilon=-1
\end{array},\right.
$$

with

$$
A=\left[(\cos \alpha-\cos q)+\frac{\tilde{r}}{2}(q \sin q-\alpha \sin \alpha)\right], \quad B=\frac{\tilde{r}}{2} q(\cos \alpha-\cos q) .
$$

\section{SM. V Bi-periodic geometry}

In this section, we briefly apply our method to the computation of the spectrum of the diffusion operator on a finite periodic geometry where the elementary block is made of two different compartments (repeated $M$ times). Such a system may model laminated steel coils in industrial processes [18, 19] or intra- and extra-cellular spaces in biology $[69,71,96]$. This is also a good example of the numerical simplifications that our method enables. The lengths of the compartments are denoted by $l_{e}$ and $l_{i}$, their diffusion coefficients by $D_{e}$ and $D_{i}$ and the barrier between the two compartments has a permeability $\kappa$ (or equivalently a resistance $r=1 / \kappa)$. For simplicity we assume reflecting boundary conditions at the outer boundaries. Let us introduce the notations

$$
\tau_{i}=l_{i}^{2} / D_{i} \quad \text { and } \quad \tau_{e}=l_{e}^{2} / D_{e}
$$

In that case, the equation (19) on the spectrum is $\mathcal{M}^{M}\left[\begin{array}{l}1 \\ 0\end{array}\right]=\epsilon\left[\begin{array}{l}1 \\ 0\end{array}\right]$, with

$$
\mathcal{M}=\left[\begin{array}{cc}
1 & r \sqrt{\lambda D_{i}} \\
0 & \sqrt{D_{i} / D_{e}}
\end{array}\right]\left[\begin{array}{cc}
\cos \left(\sqrt{\lambda \tau_{i}}\right) & \sin \left(\sqrt{\lambda \tau_{i}}\right) \\
-\sin \left(\sqrt{\lambda \tau_{i}}\right) & \cos \left(\sqrt{\lambda \tau_{i}}\right)
\end{array}\right]\left[\begin{array}{cc}
1 & r \sqrt{\lambda D_{e}} \\
0 & \sqrt{D_{e} / D_{i}}
\end{array}\right]\left[\begin{array}{cc}
\cos \left(\sqrt{\lambda \tau_{e}}\right) & \sin \left(\sqrt{\lambda \tau_{e}}\right) \\
-\sin \left(\sqrt{\lambda \tau_{e}}\right) & \cos \left(\sqrt{\lambda \tau_{e}}\right)
\end{array}\right]
$$

Because the geometry is not symmetric, $\epsilon$ is not necessary equal to \pm 1 . Moreover we have $\epsilon \eta=\sqrt{D_{e} / D_{i}}$. Following the same reasoning as in Sec. 3.1, we obtain that the solutions of Eq. (19) can be decomposed into two types: 
- the ones such that $\left[\begin{array}{l}1 \\ 0\end{array}\right]$ is an eigenvector of the transition matrix of one block, $\mathcal{M}$, from Eq. (S59). This gives the condition:

$$
r \sqrt{\lambda D_{i} D_{e}}=\sqrt{D_{i}} \cot \left(\sqrt{\lambda \tau_{e}}\right) \sin \left(\sqrt{\lambda \tau_{i}}\right)+\sqrt{D_{e}} \sin \left(\sqrt{\lambda \tau_{e}}\right) \cot \left(\sqrt{\lambda \tau_{i}}\right)
$$

Moreover, one has

$$
\epsilon=\left(\cos \left(\sqrt{\lambda \tau_{e}}\right) \cos \left(\sqrt{\lambda \tau_{i}}\right)-\sqrt{\frac{D_{i}}{D_{e}}} \sin \left(\sqrt{\lambda \tau_{e}}\right) \sin \left(\sqrt{\lambda \tau_{i}}\right)-r \sqrt{\lambda D_{i}} \cos \left(\sqrt{\lambda \tau_{e}}\right) \sin \left(\sqrt{\lambda \tau_{i}}\right)\right)^{-M}
$$

- the ones such that $\operatorname{Tr}(\mathcal{M})=2 \cos p \pi / M$, with $p=1, \ldots, M-1$, which corresponds to $\mathcal{M}^{M}=(-1)^{p} \mathcal{I}_{2}$ and thus to $\epsilon=(-1)^{p}$. This gives the equation

$$
\begin{aligned}
2 \cos p \pi / M & =2 \cos \left(\sqrt{\lambda \tau_{e}}\right) \cos \left(\sqrt{\lambda \tau_{i}}\right)-\left(\sqrt{\frac{D_{i}}{D_{e}}}+\sqrt{\frac{D_{e}}{D_{i}}}\right) \sin \left(\sqrt{\lambda \tau_{e}}\right) \sin \left(\sqrt{\lambda \tau_{i}}\right) \\
& -2 r \sqrt{\lambda}\left(\sqrt{D_{e}} \sin \left(\sqrt{\lambda \tau_{e}}\right) \cos \left(\sqrt{\lambda \tau_{i}}\right)+\sqrt{D_{i}} \cos \left(\sqrt{\lambda \tau_{e}}\right) \sin \left(\sqrt{\lambda \tau_{i}}\right)\right) \\
& +r^{2} \lambda \sqrt{D_{i} D_{e}} \sin \left(\sqrt{\lambda \tau_{e}}\right) \sin \left(\sqrt{\lambda \tau_{i}}\right), \quad p=1, \ldots, M-1 .
\end{aligned}
$$

It is interesting to compare the above equations with the analysis conducted in Sec. 2.5. Indeed, one can see that in the limit of quasi-impermeable barriers $(r \rightarrow \infty)$, Eq. (S60) yields approximately

$$
\sqrt{\lambda / D_{e}} \approx \frac{n \pi}{l_{e}}+\frac{1}{n \pi r D_{e}} \quad \text { and } \quad \sqrt{\lambda / D_{i}} \approx \frac{n \pi}{l_{i}}+\frac{1}{n \pi r D_{i}}, \quad n=1,2, \ldots,
$$

which is exactly Eq. (34) with $\zeta=1$, that is for the outer compartments. In the same way, Eq. (S62) yields approximately

$$
\begin{aligned}
& \sqrt{\lambda / D_{e}} \approx \frac{n \pi}{l_{e}}+\frac{2}{n \pi r D_{e}}+\frac{l_{e} \sqrt{D_{e} / D_{i}} X_{p}}{\left(n \pi r D_{e}\right)^{2}} \\
& \sqrt{\lambda / D_{i}} \approx \frac{n \pi}{l_{i}}+\frac{2}{n \pi r D_{i}}+\frac{l_{i} \sqrt{D_{i} / D_{e}} Y_{p}}{\left(n \pi r D_{i}\right)^{2}}
\end{aligned}
$$

where $n=1,2, \ldots$, and $X_{p}, Y_{p}$ are dimensionless coefficients which depend on the value of $\cos p \pi / M$, with $p=1, \ldots, M-1$. One recognizes the first order correction from Eq. (34) for inner compartments. The second order correction is also discussed in Eq. (2.5) and arises from the next-nearest neighbor coupling between the compartments of the same type. Therefore, in the low-permeability limit, the spectrum is made of groups of $M$ closely packed eigenvalues located around $\lambda=D_{e}\left(n \pi / l_{e}\right)^{2}$ or $\lambda=D_{i}\left(n \pi / l_{i}\right)^{2}$ : one eigenvalue is given by Eq. (S60) then the following $M-1$ eigenvalues are given by Eq. (S62). These groups correspond to eigenmodes localized inside all compartments of type " $e$ " or " $i$ ", respectively. More precisely, the first eigenvalue of each group corresponds to an eigenmode localized inside an outer compartment and the $M-1$ following eigenvalues correspond to eigenmodes localized inside all inner compartments.

Equations (S60) and (S62) "disentangle" these groups of eigenvalues, that allows one to compute very fast the spectrum of the diffusion operator for any number of repetitions $M$ and any barrier permeability. This is a major simplification of the numerical problem of the determination of the spectrum (see Sec. 2.5 and 4.1). The same remark applies to any finite periodic geometry, provided that the repeated elementary block is not too long. 


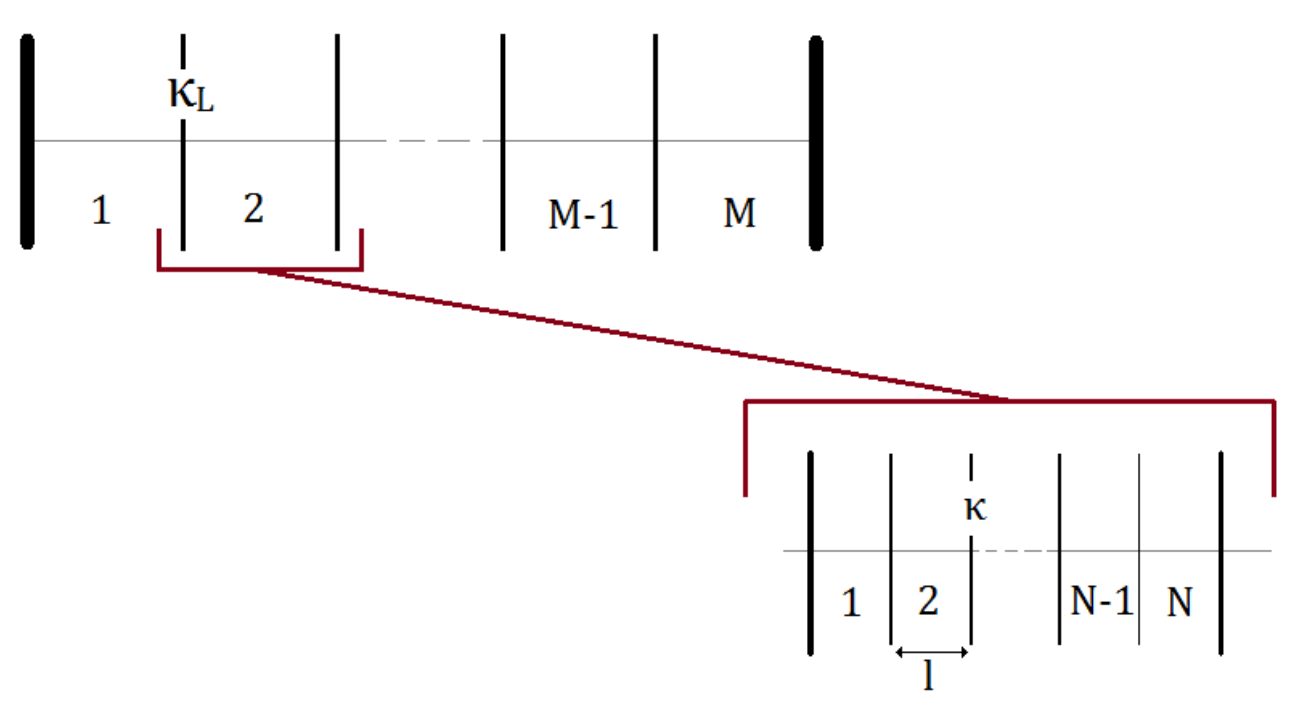

Fig. S6 Illustration of the two-scale geometry, which is a repetition of $M$ blocks of $N$ cells. All the cells have the same length $l$ and diffusion coefficient $D$ and are separated by barriers of permeability $\kappa$. The blocks are separated by barriers of permeability $\kappa_{L}$.

\section{SM. VI Two-scale geometry}

\section{SM. VI.1 Eigenmodes}

We consider again the repetition of an elementary block but without restricting ourselves to a small block. Indeed the structure is the repetition of $M$ arrays of $N$ identical cells, each array being separated from others by a "large barrier" (see Fig. S6). For simplicity we assume reflecting boundary conditions at the endpoints. The cells are of length $l$, the barriers are of permeability $\kappa$, the diffusion coefficient is $D$, and the "larger barriers" are of permeability $\kappa_{L}$. In addition to the notations (41), we introduce:

$$
\tilde{r}_{L}=1 / \tilde{\kappa}_{L}=D /\left(\kappa_{L} l\right) \quad \text { and } \quad \tilde{\rho}=\tilde{r}_{L}-\tilde{r} .
$$

Strictly speaking, $\tilde{\rho}$ may be negative, however we have in mind the opposite case where the "larger barriers" are less permeable than the inner barriers.

We have two different matrices to consider:

- the matrix associated to the microstructure is $\mathcal{M}_{1}=\left[\begin{array}{cc}1 & \tilde{r} \alpha \\ 0 & 1\end{array}\right]\left[\begin{array}{cc}\cos \alpha & \sin \alpha \\ -\sin \alpha & \cos \alpha\end{array}\right]$.

- the matrix associated to the macrostructure is $\mathcal{M}_{2}=\left[\begin{array}{cc}1 & \tilde{\rho} \alpha \\ 0 & 1\end{array}\right] \mathcal{M}_{1}{ }^{N}$.

Thanks to the formula (45), we can compute the matrix $\mathcal{M}_{2}$ :

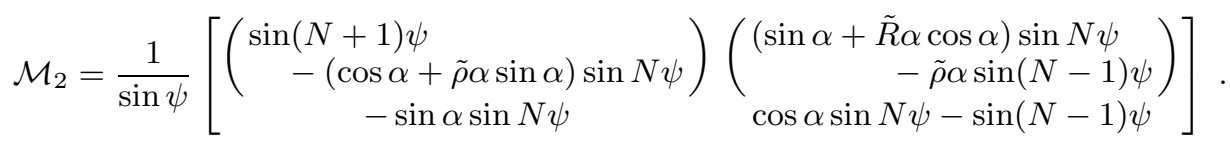

Since the geometry is symmetric, Eq. (19) of the spectrum is

$$
\mathcal{M}_{2}{ }^{M}\left[\begin{array}{l}
1 \\
0
\end{array}\right]=\epsilon\left[\begin{array}{l}
1 \\
0
\end{array}\right]
$$

with $\epsilon= \pm 1$, and by analogy with the finite periodic geometry from Sec. 3.1 we have two cases: 
$-\sin \alpha \frac{\sin N \psi}{\sin \psi}=0$ : the vector $\left[\begin{array}{l}1 \\ 0\end{array}\right]$ is an eigenvector of the matrix $\mathcal{M}_{2}$. This condition gives exactly the solutions $\alpha_{j, p}, j=0,1, \ldots$ and $p=0, \ldots, N$ (Sec. 3.1). One has $\epsilon=(-1)^{p M}$.

- The trace of the matrix $\mathcal{M}_{2}$ is $2 \cos P \pi / M$, for $P \in\{1, \ldots, M-1\}: \mathcal{M}_{2}{ }^{M}$ is plus or minus the identity matrix $\mathcal{I}_{2}$, which gives the condition:

$$
\cos N \psi-\frac{\tilde{r}}{2} \alpha \sin \alpha \frac{\sin N \psi}{\sin \psi}=\cos P \pi / M, \quad P=1, \ldots, M-1 .
$$

In this case $\epsilon=(-1)^{P}$. Again, we use a special notation for the solutions: $\alpha_{j, p, P}$, where the index $j$ means $j \pi \leq \alpha_{j, p, P}<(j+1) \pi$ and the index $p$ means $p \pi / N \leq \psi_{j, p, P}<(p+1) \pi / N$. The $P=0$ (resp., $P=M$ ) case corresponds then to the solutions for the finite periodic case $\alpha_{j, p}$ if $p$ is even (resp. if $p$ is odd).

The interpretation of the indices $j, p, P$ follows the same line of reasoning as with the simple periodic geometry: they give the intra-compartment, inter-compartment (or intra-block) and inter-block variation of the mode, respectively.

\section{SM. VI.2 Computation of the norm:}

We use again Eq. (29):

$$
\begin{aligned}
{\left[\begin{array}{ll}
0 & 1
\end{array}\right] \mathcal{T}\left[\begin{array}{l}
1 \\
0
\end{array}\right] } & =\left[\begin{array}{ll}
0 & 1
\end{array}\right]\left(\mathcal{K}_{2} \mathcal{M}^{N}\right)^{M}\left[\begin{array}{l}
1 \\
0
\end{array}\right]=\frac{\sin M \phi}{\sin \phi}\left[\begin{array}{ll}
0 & 1
\end{array}\right] \mathcal{K}_{2} \mathcal{M}^{N}\left[\begin{array}{l}
1 \\
0
\end{array}\right] \\
& =\frac{\sin M \phi}{\sin \phi} \frac{\sin N \psi}{\sin \psi}\left[\begin{array}{ll}
0 & 1
\end{array}\right] M\left[\begin{array}{l}
1 \\
0
\end{array}\right]=-\frac{\sin M \phi}{\sin \phi} \frac{\sin N \psi}{\sin \psi} \sin \alpha,
\end{aligned}
$$

where we have introduced $\phi$ defined by

$$
\cos \phi=\frac{1}{2} \operatorname{Tr}\left(\mathcal{K}_{2} \mathcal{M}^{m}\right)=\cos N \psi-\frac{\tilde{r}}{2} \alpha \sin \alpha \frac{\sin N \psi}{\sin \psi} .
$$

Now we have three cases:

1. $\sin \alpha=0$, which corresponds to $\alpha_{j, 0}$ and $\alpha_{j, N}$. One gets

$$
\beta^{2}=\frac{2}{M N l}
$$

2. $\frac{\sin N \psi}{\sin \psi}=0$, which corresponds to $\alpha_{j, p}, p=1, \ldots, N-1$. In this case we get

$$
\beta_{j, p}^{2}=\frac{2}{m l} \frac{\sin ^{2} p \pi / N}{\sin \alpha_{j, p}\left(\sin \alpha_{j, p}\left(1+\frac{\tilde{r}}{2}\right)+\frac{\tilde{r}}{2} \alpha_{j, p} \cos \alpha_{j, p}\right)} .
$$

3. $\frac{\sin M \phi}{\sin \phi}=0$, which corresponds to the general case. We use the chain rule again to compute the derivative with respect to $\alpha$ :

$$
\begin{aligned}
& \frac{\mathrm{d}}{\mathrm{d} \alpha}\left(\frac{\sin M \phi}{\sin \phi}\right)=\frac{\mathrm{d} \cos \phi}{\mathrm{d} \alpha} \frac{\mathrm{d} \phi}{\mathrm{d} \cos \phi} \frac{\mathrm{d}}{\mathrm{d} \phi}\left(\frac{\sin M \phi}{\sin \phi}\right) \\
& \frac{\mathrm{d} \cos \phi}{\mathrm{d} \alpha}=-N \frac{1-\cos N \psi \cos P \pi / M}{\sin N \psi \sin \psi}\left[\left(1+\frac{\tilde{r}}{2}\right) \sin \alpha+\frac{\tilde{r}}{2} \alpha \cos \alpha\right] \\
& +\frac{\cos N \psi-\cos P \pi / M}{\sin ^{2} \psi}\left[\frac{\sin ^{2} \alpha}{\alpha}+\frac{\tilde{r}}{2}(\alpha+\sin \alpha \cos \alpha)\right] \\
& \frac{\mathrm{d} \phi}{\mathrm{d} \cos \phi} \frac{\mathrm{d}}{\mathrm{d} \phi}\left(\frac{\sin M \phi}{\sin \phi}\right)=\left(\frac{-1}{\sin P \pi / M}\right)\left(\frac{(-1)^{P}}{\sin P \pi / M}\right) .
\end{aligned}
$$


Hence we get the normalization constant:

$$
\beta_{j, p, P}^{2}=\left.\frac{\frac{2 \sin ^{2}(P \pi / M) \sin \psi}{m l \sin \alpha \sin N \psi}}{\left[\begin{array}{c}
\frac{1-\cos N \psi \cos P \pi / M}{\sin N \psi \sin \psi}\left(\left(1+\frac{\tilde{r}}{2}\right) \sin \alpha+\frac{\tilde{r}}{2} \alpha \cos \alpha\right) \\
+\frac{\cos N \psi-\cos P \pi / M}{N \sin ^{2} \psi}\left(\frac{\sin ^{2} \alpha}{\alpha}+\frac{\tilde{r}}{2}(\alpha+\sin \alpha \cos \alpha)\right)
\end{array}\right]}\right|_{\alpha=\alpha_{j, p, P}}
$$

\section{SM. VI.3 Fourier transform}

In the same way as for the finite periodic geometry, we have only one $\mathcal{L}$ to consider, so we need to compute

$$
\begin{aligned}
& \sum_{i} e^{i k q} \mathcal{L}_{i}\left[\begin{array}{l}
a_{i}^{l} \\
b_{i}^{l}
\end{array}\right]=\mathcal{L} \sum_{i=0}^{M-1} \sum_{i=0}^{N-1} e^{i q(K N+k)} \mathcal{M}_{1}{ }^{k} \mathcal{M}_{2}{ }^{K}\left[\begin{array}{l}
1 \\
0
\end{array}\right] \\
& =\mathcal{L}\left(\mathcal{I}_{2}-e^{i q} \mathcal{M}_{1}\right)^{-1}\left(\mathcal{I}_{2}-e^{i N q} \mathcal{M}_{1}{ }^{N}\right)\left(\mathcal{I}_{2}-e^{i q N} \mathcal{M}_{2}\right)^{-1}\left(\mathcal{I}_{2}-e^{i N M q} \mathcal{M}_{2}{ }^{M}\right)\left[\begin{array}{l}
1 \\
0
\end{array}\right] .
\end{aligned}
$$

Using Eq. (S67) on the spectrum and the linearity of the comatrix operation, we get to simplify a lot the above expression:

$$
\sum_{i} e^{i k q} \mathcal{L}_{i}\left[\begin{array}{c}
a_{i}^{l} \\
b_{i}^{l}
\end{array}\right]=\left(1-(-1)^{P} e^{i N M q}\right) \frac{\operatorname{det}\left(\mathcal{I}_{2}-e^{i N q} \mathcal{M}_{1}^{N}\right)}{\operatorname{det}\left(\mathcal{I}_{2}-e^{i N q} \mathcal{M}_{2}\right)} \mathcal{L}\left(\mathcal{I}_{2}-e^{i q} \mathcal{M}_{1}\right)^{-1}\left[\begin{array}{l}
1 \\
0
\end{array}\right]
$$

And finally

$$
\int_{0}^{L} v(x) e^{i \gamma g \delta x} \mathrm{~d} x=\frac{i q l\left(1-(-1)^{P} e^{i N M q}\right) \frac{\cos N \psi_{j, p, P}-\cos N q}{\cos P \pi / M-\cos N q} \frac{\cos \alpha_{j, p, P}-\cos q}{\cos \psi_{j, p, P}-\cos q}}{q^{2}-\alpha_{j, p, P^{2}}}
$$

SM. VI.4 Complete expression of the dMRI signal

We gather the above expressions to obtain the signal as a function of $q=\gamma g \delta l$ and $t=D \Delta / l^{2}$ :

$$
\begin{gathered}
S=\frac{2(1-\cos m q)}{(m q)^{2}}+\sum_{j=1}^{\infty} \frac{4 q^{2}\left(1-(-1)^{j m} \cos m q\right)}{m^{2}\left(q^{2}-(j \pi)^{2}\right)^{2}} e^{-(j \pi)^{2} t} \\
+\sum_{j=0}^{\infty} \sum_{p=1}^{N-1} \frac{2 l q^{2}}{m} \frac{1-(-1)^{p M} \cos m q}{(\cos q-\cos p \pi / N)^{2}}\left(\frac{\cos q-\cos \alpha_{j, p}}{q^{2}-\alpha_{j, p}^{2}}\right)^{2} \beta_{j, p}^{2} e^{-\alpha_{j, p}{ }^{2} t} \\
+\sum_{j=1}^{\infty} \sum_{p=0}^{N} \sum_{P=1}^{M-1} \frac{2 m l q^{2}\left(1-(-1)^{P} \cos m q\right)}{M^{2}(\cos N q-\cos P \pi / M)^{2}}\left(\frac{\cos q-\cos \alpha_{j, p, P}}{q^{2}-\alpha_{j, p, P}{ }^{2}}\right)^{2}\left(\frac{\cos N q-\cos N \psi_{j, p, P}}{N\left(\cos q-\cos \psi_{j, p, P}\right)}\right)^{2} \\
\times \beta_{j, p, P}^{2} e^{-\alpha_{j, p, P}{ }^{2} t},
\end{gathered}
$$

where $\beta_{j, p}^{2}$ and $\beta_{j, p, P}^{2}$ are given by Eqs. (49) and (S71), respectively. 
SM. VII Limit of the dMRI signal for the periodic geometry as $\tilde{\kappa} \rightarrow 0$ and $\tilde{\kappa} \rightarrow \infty$

SM. VII.1 High-permeability limit: $\tilde{\kappa} \rightarrow \infty$

In this limit, one has:

$$
\begin{cases}\alpha_{j, p}=j \pi+p \pi / m & \text { if } j \text { is even } \\ \alpha_{j, p}=j \pi+(m-p) \pi / m & \text { if } j \text { is odd. }\end{cases}
$$

In particular, $\cos \alpha_{j, p}=\cos \psi_{j, p}$, so the expression of the signal simplifies into

$$
\begin{aligned}
S & =\frac{2(1-\cos m q)}{(m q)^{2}}+\sum_{j=1}^{\infty} \frac{4 q^{2}\left(1-(-1)^{j m} \cos m q\right)}{m^{2}\left(q^{2}-(j \pi)^{2}\right)^{2}} e^{-(j \pi)^{2} t} \\
& +\sum_{j=0}^{\infty} \sum_{p=1}^{m-1} \frac{2 l q^{2}}{m} \frac{1-(-1)^{p} \cos m q}{\left(q^{2}-\alpha_{j, p}^{2}\right)^{2}} \beta_{j, p}^{2} e^{-\alpha_{j, p}^{2} t},
\end{aligned}
$$

with $\beta_{j, p}^{2}=2 /(m l)$. Hence:

$$
S=\frac{2(1-\cos m q)}{(m q)^{2}}+\sum_{n=1}^{\infty} \frac{4 q^{2}\left(1-(-1)^{n} \cos m q\right)}{\left((m q)^{2}-(n \pi)^{2}\right)^{2}} e^{-(n \pi)^{2} t / m^{2}}
$$

which is the formula of the signal for one interval of length $L=m l$, as expected.

SM. VII.2 Low-permeability limit: $\tilde{\kappa} \rightarrow 0$

Although the result is intuitively expected, the computation is more complicated. The mathematical reason is that in the limit $\tilde{\kappa} \rightarrow 0, \alpha_{j, p}=j \pi$ so that the eigenmodes of the branch $j$ are degenerate. Using Eq. (48), one gets the expression of the signal:

$$
\begin{aligned}
S= & \frac{2(1-\cos m q)}{(m q)^{2}}+\sum_{j=1}^{\infty} \frac{4 q^{2}\left(1-(-1)^{j m} \cos m q\right)}{m^{2}\left(q^{2}-(j \pi)^{2}\right)^{2}} e^{-(j \pi)^{2} t} \\
& +\sum_{j=0}^{\infty} \sum_{p=1}^{m-1} \frac{2 l q^{2}}{m} \frac{1-(-1)^{p} \cos m q}{(\cos q-\cos p \pi / m)^{2}}\left(\frac{\cos q-(-1)^{j}}{q^{2}-(j \pi)^{2}}\right)^{2} \beta_{j, p}^{2} e^{-(j \pi)^{2} t},
\end{aligned}
$$

with

$$
\left\{\begin{array}{l}
\beta_{j, p}^{2}=\frac{2}{m l}\left(1+(-1)^{j} \cos p \pi / m\right) \quad \text { if } j>0, \\
\beta_{0, p}^{2}=\frac{1}{m l}\left(1+(-1)^{j} \cos p \pi / m\right) .
\end{array}\right.
$$

Gathering all the terms, we obtain

$$
S=S_{0}(q) \frac{2(1-\cos q)^{2}}{m^{2} q^{2}}+\frac{4 q^{2}}{m^{2}} \sum_{j=1}^{\infty} S_{j}(q) \frac{\left(1-(-1)^{j} \cos q\right)^{2}}{\left(q^{2}-(j \pi)^{2}\right)^{2}} e^{-(j \pi)^{2} t}
$$

with

$$
S_{j}(q)=\sum_{p=0}^{m} \frac{\left(1-(-1)^{p} \cos m q\right)\left(1+(-1)^{j} \cos p \pi / m\right)}{(\cos q-\cos p \pi / m)^{2}\left(1+\theta_{p}\right)}, j=0,1, \ldots
$$

where $\theta_{p}=1$ if $p=0$ or $m$, and $\theta_{p}=0$ otherwise. To compute $S_{j}(q)$, we introduce the following polynomial:

$$
\mathcal{P}(X)=\prod_{p=0}^{m}(X-\cos p \pi / m)
$$


The analysis of its roots and degree leads to the following formula:

$$
\mathcal{P}(\cos q)=\mathcal{N} \sin (m q) \sin q
$$

where $\mathcal{N}$ is an unknown proportionality coefficient whose value is not needed in the following. This allows us to compute

$$
\begin{aligned}
& \mathcal{P}^{\prime}(\cos q)=\left(\frac{-1}{\sin q}\right) \mathcal{N}(m \cos (m q) \sin q+\sin (m q) \cos q) \\
& \mathcal{P}^{\prime}(\cos p \pi / m)=\mathcal{N} m(-1)^{p+1}\left(1+\theta_{p}\right)
\end{aligned}
$$

Now we use the standard partial fraction expansion formula, for any polynomial $\mathcal{Q}$ such that $\operatorname{deg} \mathcal{Q} \leq$ $\operatorname{deg} \mathcal{P}$ :

$$
\frac{\mathcal{Q}(X)}{\mathcal{P}(X)}=C+\sum_{p=0}^{m} \frac{\mathcal{Q}(\cos p \pi / m)}{\mathcal{P}^{\prime}(\cos p \pi / m)(X-\cos p \pi / m)},
$$

where prime denotes the derivative with respect to $X$ and $C$ is a constant. With the polynomial $\mathcal{R}(\cos q)=$ $\cos m q$, we get according to Eq. (S84)

$$
\begin{aligned}
S_{j}(q) & =\mathcal{N} m\left[\left(\frac{\mathcal{R}(X)\left(1+(-1)^{j} X\right)}{\mathcal{P}(X)}\right)^{\prime}-\mathcal{R}(X)\left(\frac{1+(-1)^{j} X}{\mathcal{P}(X)}\right)^{\prime}\right]_{X=\cos q} \\
& =\mathcal{N} m \mathcal{R}^{\prime}(\cos q) \frac{1+(-1)^{j} \cos q}{\mathcal{P}(\cos q)}
\end{aligned}
$$

Computing the derivative of $\mathcal{R}$ and using Eq. (S81), one finally gets

$$
S_{j}(q)=\frac{m^{2}}{1-(-1)^{j} \cos q}
$$

Now we come back to Eq. (S78), which yields

$$
S=\frac{2(1-\cos q)}{q^{2}}+\sum_{j=1}^{\infty} \frac{4 q^{2}\left(1-(-1)^{j} \cos q\right)}{\left(q^{2}-(j \pi)^{2}\right)^{2}} e^{-(j \pi)^{2} t}
$$

which is the expected formula of the signal for one interval of length $l$.

\section{SM. VIII Expansions for $\alpha_{j, p}$ for the periodic geometry}

Low-permeability limit: $\tilde{\kappa} \rightarrow 0$ In this case we rewrite Eq. (44) as $\alpha \sin \alpha=2 \tilde{\kappa}(\cos \alpha-\cos \psi)$. We start with the branch $j=0$. Let us write $\alpha=u \sqrt{2 \tilde{\kappa}(1-\cos \psi)}$. Then

$$
\begin{aligned}
\alpha \sin \alpha & =2 \tilde{\kappa}(1-\cos \psi) u^{2}\left(1-\frac{1}{3} \tilde{\kappa}(1-\cos \psi) u^{2}\right)+O\left(\tilde{\kappa}^{3}\right), \\
(\cos \alpha-\cos \psi) & =(1-\cos \psi)-\tilde{\kappa}(1-\cos \psi) u^{2}+O\left(\tilde{\kappa}^{2}\right),
\end{aligned}
$$

from which we derive

$$
\alpha_{0, p}=2 \tilde{\kappa}^{1 / 2} \sin (p \pi / 2 m)-\tilde{\kappa}^{3 / 2}\left(\sin (p \pi / 2 m)-\frac{2}{3} \sin ^{2}(p \pi / 2 m)\right)+O\left(\tilde{\kappa}^{5 / 2}\right) .
$$

Now, if $\alpha=j \pi+\epsilon$, one has

$$
\alpha \sin \alpha=(-1)^{j}\left(j \pi \epsilon+\epsilon^{2}+O\left(\epsilon^{3}\right)\right), \quad(\cos \alpha-\cos \psi)=(-1)^{j}\left(1-(-1)^{j} \cos \psi+O\left(\epsilon^{2}\right)\right)
$$


which gives

$$
\alpha_{j, p}= \begin{cases}j \pi+\frac{4 \tilde{\kappa}}{j \pi} \sin ^{2}(p \pi / 2 m)-\frac{(4 \tilde{\kappa})^{2}}{(j \pi)^{3}} \sin ^{4}(p \pi / 2 m)+O\left(\tilde{\kappa}^{3}\right) & \text { if } j \text { is even } \\ j \pi+\frac{4 \tilde{\kappa}}{j \pi} \sin ^{2}((m-p) \pi / 2 m)-\frac{(4 \tilde{\kappa})^{2}}{(j \pi)^{3}} \sin ^{4}((m-p) \pi / 2 m)+O\left(\tilde{\kappa}^{3}\right) & \text { if } j \text { is odd. }\end{cases}
$$

This is consistent with the idea that at very low permeability the compartments become independent so that $\alpha_{j, p}$ (with $p=1, \ldots, m-1$ ) are identical and equal to $j \pi$. One notices that the deviation from this limit decreases with $j$ which is consistent with previous observations (Fig. 3).

High permeability limit: $\tilde{r} \rightarrow 0$ Again, we start with the $j=0$ branch. Let us write $\alpha=\psi-u$. Then we have the equations:

$$
\begin{aligned}
\cos \alpha & =\cos \psi\left(1-\frac{u^{2}}{2}+O\left(u^{4}\right)\right)+\sin \psi\left(u+O\left(u^{3}\right)\right), \\
\alpha \sin \alpha & =\psi \sin \psi+u \sin \psi+u \psi \cos \psi+O\left(u^{3}\right)
\end{aligned}
$$

which yield

$$
\alpha_{0, p}=\frac{p \pi}{m}\left(1-\frac{\tilde{r}}{2}+\frac{\tilde{r}^{2}}{4}\left[1+\frac{p \pi / m}{2 \tan (p \pi / m)}\right]+O\left(\tilde{r}^{3}\right)\right) .
$$

For the other branches, the computations are similar:

$$
\alpha_{j, p}= \begin{cases}(j \pi+p \pi / m)\left(1-\frac{\tilde{r}}{2}+\frac{\tilde{r}^{2}}{4}\left[1+\frac{j \pi+p \pi / m}{2 \tan (p \pi / m)}\right]\right)+O\left(\tilde{r}^{3}\right) & j \text { even } \\ (j \pi+(m-p) \pi / m)\left(1-\frac{\tilde{r}}{2}+\frac{\tilde{r}^{2}}{4}\left[1+\frac{j \pi+(m-p) \pi / m}{2 \tan ((m-p) \pi / m)}\right]\right)+O\left(\tilde{r}^{3}\right) & j \text { odd }\end{cases}
$$

Again, the interpretation is quite clear. When the permeability is very high, $\tilde{r} \rightarrow 0$ and the $\alpha_{j, p}$ approach the solutions for one interval of length $m l$, for which $\alpha_{n}=n \pi / m(n=0,1, \ldots)$. Consistently with the above low-permeability regime, the deviation from the limit $\tilde{\kappa}=\infty$ increases with $j$.

\section{References}

1. H. S. Carslaw, J. C. Jaeger, Conduction of Heat in Solids, Clarendon Press, 1959.

2. J. Crank, The Mathematics of Diffusion, Clarendon Press, 1975.

3. D. S. Grebenkov, B.-T. Nguyen, Geometrical Structure of Laplacian Eigenfunctions, SIAM Review 55 (2013) 601-667.

4. A. Lejay, G. Pichot, Simulating diffusion processes in discontinuous media: A numerical scheme with constant time steps, Journal of Computational Physics 231 (2012) 7299 - 7314.

5. A. Lejay, Estimation of the mean residence time in cells surrounded by semi-permeable membranes by a Monte Carlo method, Research Report RR-8709, Inria Nancy - Grand Est (Villers-lès-Nancy, France) ; INRIA, 2015. URL: https://hal.inria.fr/hal-01140960.

6. R. Hickson, S. Barry, G. Mercer, H. Sidhu, Finite difference schemes for multilayer diffusion, Mathematical and Computer Modelling 54 (2011) $210-220$.

7. J.-P. Diard, N. Glandut, C. Montella, J.-Y. Sanchez, One layer, two layers, etc. An introduction to the EIS study of multilayer electrodes. Part 1: Theory, Journal of Electroanalytical Chemistry 578 (2005) $247-257$.

8. V. Freger, Diffusion impedance and equivalent circuit of a multilayer film, Electrochemistry Communications 7 (2005) 957 - 961.

9. R. Ngameni, P. Millet, Derivation of the diffusion impedance of multi-layer cylinders. Application to the electrochemical permeation of hydrogen through $\mathrm{Pd}$ and $\mathrm{PdAg}$ hollow cylinders, Electrochimica Acta 131 (2014) 52 - 59. Electrochemical Impedance Spectroscopy. 
10. G. L. Graff, R. E. Williford, P. E. Burrows, Mechanisms of vapor permeation through multilayer barrier films: Lag time versus equilibrium permeation, Journal of Applied Physics 96 (2004) 18401849.

11. Y. Gurevich, I. Lashkevich, G. G. de la Cruz, Effective thermal parameters of layered films: An application to pulsed photothermal techniques, International Journal of Heat and Mass Transfer 52 (2009) $4302-4307$.

12. N. Muoz Aguirre, G. Gonzlez de la Cruz, Y. Gurevich, G. Logvinov, M. Kasyanchuk, Heat Diffusion in Two-Layer Structures: Photoacoustic Experiments, physica status solidi (b) 220 (2000) 781-787.

13. P. Grossel, F. Depasse, Alternating heat diffusion in thermophysical depth profiles: multilayer and continuous descriptions, Journal of Physics D: Applied Physics 31 (1998) 216.

14. X. Lu, P. Tervola, Transient heat conduction in the composite slab-analytical method, Journal of Physics A: Mathematical and General 38 (2005) 81.

15. X. Lu, P. Tervola, M. Viljanen, Transient analytical solution to heat conduction in composite circular cylinder, International Journal of Heat and Mass Transfer 49 (2006) $341-348$.

16. F. de Monte, Transient heat conduction in one-dimensional composite slab. A natural analytic approach, International Journal of Heat and Mass Transfer 43 (2000) $3607-3619$.

17. S. Barbaro, C. Giaconia, A. Orioli, A computer oriented method for the analysis of non steady state thermal behaviour of buildings, Building and Environment 23 (1988) $19-24$.

18. W. Yuen, Transient temperature distribution in a multilayer medium subject to radiative surface cooling, Applied Mathematical Modelling 18 (1994) 93 - 100.

19. R. Hickson, S. Barry, G. Mercer, Critical times in multilayer diffusion. Part 1: Exact solutions, International Journal of Heat and Mass Transfer 52 (2009) 5776 - 5783.

20. R. Hickson, S. Barry, G. Mercer, Critical times in multilayer diffusion. Part 2: Approximate solutions, International Journal of Heat and Mass Transfer 52 (2009) $5784-5791$.

21. C. D. Shackelford, Laboratory diffusion testing for waste disposal A review, Journal of Contaminant Hydrology 7 (1991) $177-217$.

22. G. Liu, L. Barbour, B. C. Si, Unified Multilayer Diffusion Model and Application to Diffusion Experiment in Porous Media by Method of Chambers, Environmental Science \& Technology 43 (2009) 2412-2416.

23. C. D. Shackelford, S. M. Moore, Fickian diffusion of radionuclides for engineered containment barriers: Diffusion coefficients, porosities, and complicating issues, Engineering Geology 152 (2013) 133 - 147.

24. S. R. Yates, S. K. Papiernik, F. Gao, J. Gan, Analytical solutions for the transport of volatile organic chemicals in unsaturated layered systems, Water Resources Research 36 (2000) 1993-2000.

25. R. A. Siegel, A Laplace transform technique for calculating diffusion time lags, Journal of Membrane Science 26 (1986) $251-262$.

26. G. Pontrelli, F. de Monte, Mass diffusion through two-layer porous media: an application to the drug-eluting stent, International Journal of Heat and Mass Transfer 50 (2007) 3658 - 3669.

27. H. Todo, T. Oshizaka, W. R. Kadhum, K. Sugibayashi, Mathematical model to predict skin concentration after topical application of drugs, Pharmaceutics 5 (2013) 634-651.

28. D. Mantzavinos, M. Papadomanolaki, Y. Saridakis, A. Sifalakis, Fokas transform method for a brain tumor invasion model with heterogeneous diffusion in $1+1$ dimensions, Applied Numerical Mathematics 104 (2016) 47 - 61. Fifth International Conference on Numerical Analysis Recent Approaches to Numerical Analysis: Theory, Methods and Applications (NumAn 2012), held in Ioannina Sixth International Conference on Numerical Analysis Recent Approaches to Numerical Analysis: Theory, Methods and Applications (NumAn 2014), held in Chania, in memory of Theodore S. Papatheodorou.

29. J. Canosa, R. G. D. Oliveira, A new method for the solution of the Schrödinger equation, J. Comput. Phys. 5 (1970) $188-207$.

30. S. Pruess, Estimating the eigenvalues of Sturm-Liouville problems by approximating the differential equation, SIAM J. Numer. Anal. 10 (1973) 55-68.

31. S. Pruess, High order approximations to Sturm-Liouville eigenvalues, Numer. Math. 24 (1975) 241-247.

32. M. Marletta, J. D. Pryce, Automatic solution of Sturm-Liouville problems using the pruess method, Journal of Computational and Applied Mathematics 39 (1992) 57 - 78. 
33. S. Pruess, C. T. Fulton, Mathematical software for Sturm-Liouville problems, ACM Trans. Math. Software 19 (1993) 360-376.

34. D. W. Hahn, M. N. Ozisik, One-Dimensional Composite Medium, John Wiley \& Sons, Inc., 2012 , pp. 393-432. URL: http://dx.doi.org/10.1002/9781118411285.ch10. doi:10.1002/9781118411285. ch10.

35. M. Mikhailov, M. N. Ozisik, Unified Analysis and Solutions of Heat and Mass Diffusion, John Wiley \& Sons, 1984.

36. B. Gaveau, M. Okada, T. Okada, Second order differential operators and Dirichlet integrals with singular coefficients, Tohoku Math. J. 39 (1987) 465-504.

37. E. Carr, I. Turner, A semi-analytical solution for multilayer diffusion in a composite medium consisting of a large number of layers, Applied Mathematical Modelling 40 (2016) $7034-7050$.

38. D. S. Grebenkov, Pulsed-gradient spin-echo monitoring of restricted diffusion in multilayered structures, Journal of Magnetic Resonance 205 (2010) 181 - 195.

39. I. M. Sokolov, Ito, Stratonovich, Hänggi and all the rest: The thermodynamics of interpretation, Chem. Phys. 375 (2010) 359 - 363. Stochastic processes in Physics and Chemistry (in honor of Peter Hnggi).

40. H. W. de Haan, M. V. Chubynsky, G. W. Slater, Monte-Carlo approaches for smiluating a particle at a diffusivity interface and the "Ito-Stratonovich dilemma", ArXiv e-prints (2012).

41. P. Hänggi, Stochastic processe I: Asymptotic behaviour and symmetries, Helv. Phys. Acta 51 (1978) 183-201.

42. P. Hänggi, Connection between deterministic and stochastic descriptions of nonlinear systems, Helv. Phys. Acta 53 (1980) 491-496.

43. P. Hänggi, H. Thomas, Stochastic processes: Time evolution, symmetries and linear response, Phys. Rep. 88 (1982) $207-319$.

44. Y. L. Klimontovich, Ito, Stratonovich and kinetic forms of stochastic equations, Physica A 163 (1990) $515-532$.

45. Y. L. Klimontovich, Nonlinear Brownian motion, Phys. Usp. 37 (1994) 737.

46. R. Hickson, S. Barry, H. Sidhu, G. Mercer, Critical times in single-layer reaction diffusion, International Journal of Heat and Mass Transfer 54 (2011) 2642 - 2650.

47. R. I. Hickson, S. I. Barry, H. S. Sidhu, G. N. Mercer, A comparison of critical time definitions in multilayer diffusion, The ANZIAM Journal 52 (2011) 333358.

48. J. Miller, P. Weaver, Temperature profiles in composite plates subject to time-dependent complex boundary conditions, Composite Structures 59 (2003) $267-278$.

49. M. Fukuda, H. Kawai, Diffusion of low molecular weight substances into a fiber with skin-core structurerigorous solution of the diffusion in a coaxial cylinder of multiple components, Polymer Engineering \& Science 34 (1994) 330-340.

50. M. Fukuda, H. Kawai, Diffusion of low molecular weight substances into a laminar film. I: Rigorous solution of the diffusion equation in a composite film of multiple layers, Polymer Engineering \& Science 35 (1995) 709-721.

51. D. S. Grebenkov, J.-F. Rupprecht, The escape problem for mortal walkers, The Journal of Chemical Physics 146 (2017) 084106.

52. B. Meerson, S. Redner, Mortality, redundancy, and diversity in stochastic search, Phys. Rev. Lett. 114 (2015) 198101.

53. S. B. Yuste, E. Abad, K. Lindenberg, Exploration and trapping of mortal random walkers, Phys. Rev. Lett. 110 (2013) 220603.

54. A. Biess, E. Korkotian, D. Holcman, Barriers to Diffusion in Dendrites and Estimation of Calcium Spread Following Synaptic Inputs, PLOS Computational Biology 7 (2011) 1-14.

55. S. Carranza, D. Paul, R. Bonnecaze, Design formulae for reactive barrier membranes, Chemical Engineering Science 65 (2010) 1151 - 1158.

56. B. Gray, J. Dewynne, M. Hood, G. Wake, R. Weber, Effect of deposition of combustible matter onto electric power cables, Fire Safety Journal 16 (1990) $459-467$.

57. A. Okubo, S. A. Levin, Diffusion and Ecological Problems: Modern Perspectives, Springer, New York, NY, 2001. 
58. A. B. Mann, A. J. Gavens, M. E. Reiss, D. V. Heerden, G. Bao, T. P. Weihs, Modeling and characterizing the propagation velocity of exothermic reactions in multilayer foils, Journal of Applied Physics 82 (1997) 1178-1188.

59. J.-C. Gachon, A. Rogachev, H. Grigoryan, E. Illarionova, J.-J. Kuntz, D. Kovalev, A. Nosyrev, N. Sachkova, P. Tsygankov, On the mechanism of heterogeneous reaction and phase formation in $\mathrm{Ti} / \mathrm{Al}$ multilayer nanofilms, Acta Materialia 53 (2005) 1225 - 1231.

60. P. T. Callaghan, Principles of Nuclear Magnetic Resonance Microscopy, 1st ed., Clarendon Press, 1991.

61. W. Price, NMR Studies of Translational Motion: Principles and Applications, Cambridge Molecular Science, 2009.

62. D. S. Grebenkov, NMR survey of reflected Brownian motion, Rev. Mod. Phys. 79 (2007) 1077-1137.

63. V. G. Kiselev, Fundamentals of diffusion MRI physics, NMR in Biomedicine 30 (2017) n/a-n/a.

64. J. E. Tanner, E. O. Stejskal, Restricted Self-Diffusion of Protons in Colloidal Systems by the PulsedGradient, Spin-Echo Method, The Journal of Chemical Physics 49 (1968) 1768-1777.

65. P. T. Callaghan, A. Coy, T. P. J. Halpin, D. MacGowan, K. J. Packer, F. O. Zelaya, Diffusion in porous systems and the influence of pore morphology in pulsed gradient spin-echo nuclear magnetic resonance studies, The Journal of Chemical Physics 97 (1992) 651-662.

66. A. Coy, P. T. Callaghan, Pulsed gradient spin echo nuclear magnetic resonance for molecules diffusing between partially reflecting rectangular barriers, The Journal of Chemical Physics 101 (1994) 45994609.

67. P. Callaghan, Pulsed-Gradient Spin-Echo NMR for Planar, Cylindrical, and Spherical Pores under Conditions of Wall Relaxation, Journal of Magnetic Resonance, Series A 113 (1995) 53 - 59.

68. J. E. Tanner, Transient diffusion in a system partitioned by permeable barriers. Application to NMR measurements with a pulsed field gradient, The Journal of Chemical Physics 69 (1978) 1748-1754.

69. P. W. Kuchel, C. J. Durrant, Permeability Coefficients from NMR q-Space Data: Models with Unevenly Spaced Semi-permeable Parallel Membranes, Journal of Magnetic Resonance 139 (1999) $258-272$.

70. J. G. Powles, M. J. D. Mallett, G. Rickayzen, W. A. B. Evans, Exact analytic solutions for diffusion impeded by an infinite array of partially permeable barriers, Proceedings of the Royal Society of London A: Mathematical, Physical and Engineering Sciences 436 (1992) 391-403.

71. E. G. Novikov, D. van Dusschoten, H. V. As, Modeling of Self-Diffusion and Relaxation Time NMR in Multi-Compartment Systems, Journal of Magnetic Resonance 135 (1998) 522 - 528.

72. A. Sukstanskii, D. Yablonskiy, J. Ackerman, Effects of permeable boundaries on the diffusionattenuated MR signal: insights from a one-dimensional model, Journal of Magnetic Resonance 170 (2004) $56-66$.

73. D. S. Grebenkov, D. V. Nguyen, J.-R. Li, Exploring diffusion across permeable barriers at high gradients. I. Narrow pulse approximation, Journal of Magnetic Resonance 248 (2014) 153 - 163.

74. D. S. Grebenkov, Exploring diffusion across permeable barriers at high gradients. II. Localization regime, Journal of Magnetic Resonance 248 (2014) 164 - 176.

75. D. S. Novikov, E. Fieremans, J. H. Jensen, J. A. Helpern, Random walks with barriers, Nat. Phys. 7 (2011) 508-514.

76. D. S. Novikov, J. H. Jensen, J. A. Helpern, E. Fieremans, Revealing mesoscopic structural universality with diffusion, Proceedings of the National Academy of Sciences 111 (2014) 5088-5093.

77. S. Redner, A Guide to First-Passage Processes, Cambridge University Press, 2001.

78. R. Metzler, G. Oshanin, S. Redner, First-passage phenomena and their applications, World Scientific Publishing, 2014.

79. D. Holcman, Z. Schuss, The Narrow Escape Problem, SIAM Review 56 (2014) 213-257.

80. D. S. Grebenkov, Universal Formula for the Mean First Passage Time in Planar Domains, Phys. Rev. Lett. 117 (2016) 260201.

81. J.-F. Rupprecht, O. Bénichou, D. S. Grebenkov, R. Voituriez, Exit Time Distribution in Spherically Symmetric Two-Dimensional Domains, Journal of Statistical Physics 158 (2015) 192-230.

82. D. S. Grebenkov, Laplacian eigenfunctions in NMR. I. A numerical tool, Concepts in Magnetic Resonance Part A 32A (2008) 277-301. 
83. D. S. Grebenkov, B. Helffer, R. Henry, The Complex Airy Operator on the Line with a Semipermeable Barrier, SIAM Journal on Mathematical Analysis 49 (2017) 1844-1894.

84. F. Crick, Diffusion in Embryogenesis, Nature 225 (1970) 420.

85. S. Alexander, J. Bernasconi, W. R. Schneider, R. Orbach, Excitation dynamics in random onedimensional systems, Rev. Mod. Phys. 53 (1981) 175-198.

86. Y. G. Sinai, The Limiting Behavior of a One-Dimensional Random Walk in a Random Medium, Theory of Probability \& Its Applications 27 (1983) 256-268.

87. J. Bernasconi, W. R. Schneider, Diffusion in a one-dimensional lattice with random asymmetric transition rates, Journal of Physics A: Mathematical and General 15 (1982) L729.

88. M. Azbel, Diffusion: A Layman's approach and its applications to one-dimensional random systems, Solid State Communications 43 (1982) 515 - 517.

89. B. Derrida, Velocity and diffusion constant of a periodic one-dimensional hopping model, Journal of Statistical Physics 31 (1983) 433-450.

90. S. H. Noskowicz, I. Goldhirsch, Average versus Typical Mean First-Passage Time in a Random Random Walk, Phys. Rev. Lett. 61 (1988) 500-502.

91. P. Le Doussal, First-passage time for random walks in random environments, Phys. Rev. Lett. 62 (1989) 3097-3097.

92. K. P. N. Murthy, K. W. Kehr, Mean first-passage time of random walks on a random lattice, Phys. Rev. A 40 (1989) 2082-2087.

93. K. W. Kehr, K. P. N. Murthy, Distribution of mean first-passage times in random chains due to disorder, Phys. Rev. A 41 (1990) 5728-5730.

94. M. Raykin, First-passage probability of a random walk on a disordered one-dimensional lattice, Journal of Physics A: Mathematical and General 26 (1993) 449.

95. P. Le Doussal, C. Monthus, D. S. Fisher, Random walkers in one-dimensional random environments: Exact renormalization group analysis, Phys. Rev. E 59 (1999) 4795-4840.

96. E. Fieremans, D. S. Novikov, J. H. Jensen, J. A. Helpern, Monte Carlo study of a two-compartment exchange model of diffusion, NMR in Biomedicine 23 (2010) 711-724. 